\title{
Traglast von Stäben mit dünnwandigen offenen Querschnitten
}

\section{Working Paper}

\section{Author(s):}

Grob, Josef

Publication date:

1975

Permanent link:

https://doi.org/10.3929/ethz-a-000165959

Rights / license:

In Copyright - Non-Commercial Use Permitted

Originally published in:

Bericht / Institut für Baustatik und Konstruktion ETH Zürich 56 
Traglast von Stäben mit dünnwandigen offenen Querschnitten

Josef Grob 
(C) Birkhäuser Verlag Basel und Stuttgart, 1975

ISBN 3-7643-0795-1 


\title{
Traglast von Stäben mit dünnwandigen offenen Querschnitten
}

\author{
von \\ Dr. sc. techn. Josef Grob \\ Institut für Baustatik und Konstruktion \\ Eidgenössische Technische Hochschule Zürich
}


2. GRUNDLAGEN 3

2.1 Voraussetzungen 3

2.2 Gleichgewichtsbedingungen 5

2.3 Kinematische Bedingungen 16

2.4 Verzweigte offene Querschnitte 24

3. BESTIMMUNG DER SCHNITTKRAEFTE 25

3.1 Elastizitätstheorie 25

3.2 Plastizitätstheorie 31

4. PLASTISCHER WIDERSTAND VON QUERSCHNITTEN AUS HOMOGENEN ISOTROPEN MATERIALIEN 35

4.1 Bruchmodell und Materialverhalten 35

4.2 Statische Methode 39

4.3 Kinematische Methode 43

4.4 Numerisches Beispiel 49

5. PLASTISCHER WIDERSTAND VON STAHLBETON- UND SPANNBETONQUERSCHNITTEN 53

5.1 Bruchmodell und Materialverhalten 53

5.2 Statische Methode 62

5.3 Kinematische Methode 66

5.4 Dualität zwischen statischer und kinematischer Methode 70

5.5 Bruchmechanismen 75

5.5 .1 Längsmechanismus $\quad 77$

5.5 .2 Schubmechanismus 83

5.5.3 Kombinierter Mechanismus 86

5.6 Praktische Traglastbestimmung 88

5.6.1 Prinzipielles Vorgehen 88

5.6 .2 Berechnungsmethode 89

5.6.3 Numerisches Beispiel 98

5.7 Anwendungsgrenzen $\quad 103$ 
BEZEICHNUNGEN 
Der plastische Querschnittswiderstand und die Traglast von Balken mit dünnwandigen offenen Querschnitten unter kombinierter Beanspruchung ist bis heute kaum untersucht worden. In der vorliegenden Arbeit, welche als Dissertation ausgearbeitet wurde, wendet Herr Grob konsequent die Plastizitätstheorie (elastisches-idealplastisches Material) auf dieses Problem an. Es wurden sowohl Träger aus Stahl, Stahlbeton und Spannbeton untersucht.

Wir hoffen, dass die Ergebnisse nicht nur praktische Anwendung finden werden, sondern die ausgearbeitete Methode auch Anregungen vermittelt für weitere Arbeiten über die Traglast von Trägern unter kombinierter Beanspruchung, Biegung, Normalkraft, Querkraft und Torsion. 
Bei der Bemessung eines Tragwerkes muss sich der Ingenieur Gedanken über das Materialverhalten des gewählten Baustoffes machen. Er muss die mechanischen Eigenschaften, die für das zu lösende Problem wesentlich sind, erkennen und von den Berechnungsverfahren dasjenige auswählen, welches diese Materialeigenschaften wenigstens näherungsweise zu beschreiben vermag. Den heute gebräuchlichen Berechnungsverfahren ist im allgemeinen eines der folgenden idealisierten Stoffgesetze zugrunde gelegt.

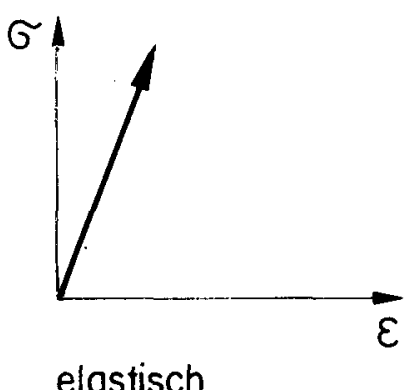

elastisch

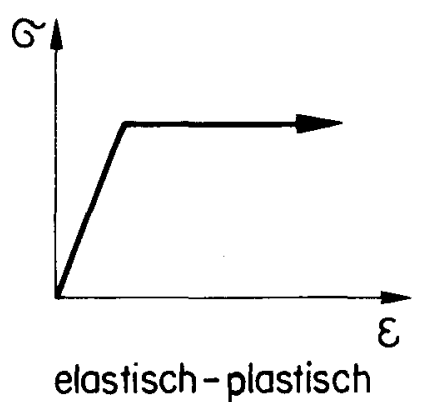

elastisch-plastisch

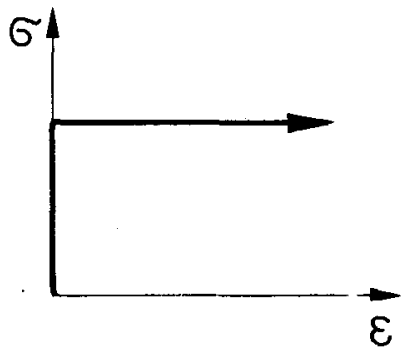

starr-plastisch

Bild 1

Für ideal-elastische Materialien ist das Tragverhalten von Stäben mit dünnwandigen offenen Querschnitten bekannt. Allerdings verhalten sich die wichtigsten Baustaffe nur für genügend kleine Spannungen und Dehnungen wie elastische Körper, was dazu führt, dass der Elastizitätstheorie Grenzen gesetzt sind. Die Verwendung eines elastischen stoffgesetzes ist nur dort sinnvoll, wo die Elastizitätsgrenze nicht überschritten wird.

Das Ziel der vorliegenden Arbeit besteht darin, eine allgemein gültige Theorie für die Berechnung der plastischen Traglast von Stäben mit dünnwandigen offenen Querschnitten zu entwickeln. Es interessiert demnach das Verhalten oberhalb des elastischen Bereiches, was eine Anwendung der reinen Elastizitätstheorie von vorne herein ausschliesst. Für zähe, duktile Materialien empfiehlt sich die Annahme eines elastisch-plastischen oder eines starrplastischen Stoffgesetzes. Wird den Berechnungen ein elastisch-plastisches Materialverhalten zugrunde gelegt, so erhält man neben dem Spannungs- auch den Verformungszustand. In allen jenen Fällen, wo die Verformungen keinen spürbaren Einfluss auf das Gleichgewicht ausüben, ist die Annahme eines elastisch-plastischen Baustoffes jedoch nicht nötig, da die Verwendung des einfacheren starr-plastischen Stoffgesetzes zur gleichen Traglast führt. Deshalb wird im folgenden ein starr-plastisches Materialverhalten zur Berechnung der Traglast von Stäben mit dünnwandigen offenen Querschnitten voraus- 
gesetzt. Die Möglichkeit einer relativ einfachen und übersichtlichen Beistimung der Traglast, welche meist das entscheidende Kriterium für die Sicherheit eines Tragwerkes darstellt, ist damit gegeben. 


\subsection{Voraussetzungen}

Die hier gemachten Voraussetzungen für die Berechnung der Traglast von Stäben mit dünnwandigen offenen Querschnitten beinhalten im wesentlichen die Annahmen der Plastizitätstheorie und der Stabtheorie bei Beschränkung auf die Theorie 1. Ordnung.

1. Die Querschnittsform bleibt erhalten.

2. Die Querschnittsabmessungen sind klein im Vergleich zur Stablänge.

3. Die Deformationen sind klein, sie haben keinen Einfluss auf die Schnittkräfte.

4. Die Stabaxe ist stückweise gerade.

5. Der Querschnitt ist stückweise konstant.

6. Der Querschnitt ist dünnwandig, die Plattenwirkung ist klein im Vergleich zur Scheibenwirkung.

7. Es wird ein starr-idealplastisches Materialverhalten angenommen.

8. Die Lasten werden proportional bis zum Erreichen der Traglast gesteigert.

Die Anwendung der Stabtheorie verlangt die Erhaltung der Querschnittsform. Diese Voraussetzung ist gerechtfertigt, wenn die im Quersinn anfallende Beanspruchung ohne wesentliche Verformung der Querschnitte aufgenommen werden kann, was durch eine geeignete Ausbildung der Querschnitte oder den Einbau von Querschotten bzw. Queraussteifungen zu gewährleisten ist. Kann die Erhaltung der Querschnittsform nicht mehr garantiert werden, so ist ein Extremfall denkbar, bei dem sich der Querschnitt widerstandslos verformen lässt. Solche Tragwerke werden als Gelenkfaltwerke bezeichnet. Auf deren Berechnung wird hier nicht eingegangen, es sei lediglich auf den diese Arbeit ergänzenden Versuchsbericht [12] hingewiesen, in welchem ein einfacher Weg zur Bestimmung der Traglast von Stahlbeton- und Spannbetonfaltwerken angegeben ist.

Die vorausgesetzte Dünnwandigkeit der Querschnitte erlaubt eine Vernachlässigung der Plattenwirkung, die im Verhältnis zur Scheibenwirkung einen nur unbedeutenden Einfluss hat. Damit ist auch gesagt, dass die St.Venant'sche Torsion, die bei stäben mit dünnwandigen offenen Querschnitten den Verwindungsanteil der Plattenwirkung berücksichtigt, in dieser Arbeit vernachlässigt wird. 


\section{Scheibenwirkung}

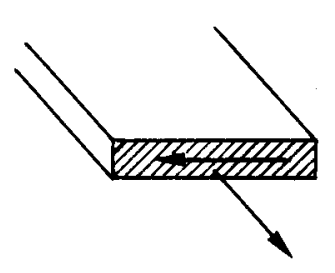

\section{Plattenwirkung}

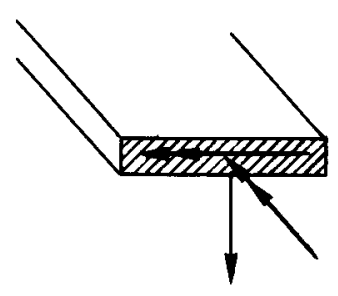

(vernochlässigt)

Allen Berechnungen wird ein rechtsdrehendes, kartesisches Koordinatensystem zugrunde gelegt, dessen $x$-Axe in Stablängsrichtung weist. Zudem wird eine Querschnittskoordinate eingeführt, welche entlang der Mittellinie des dünnwandigen Querschnittes verläuft.
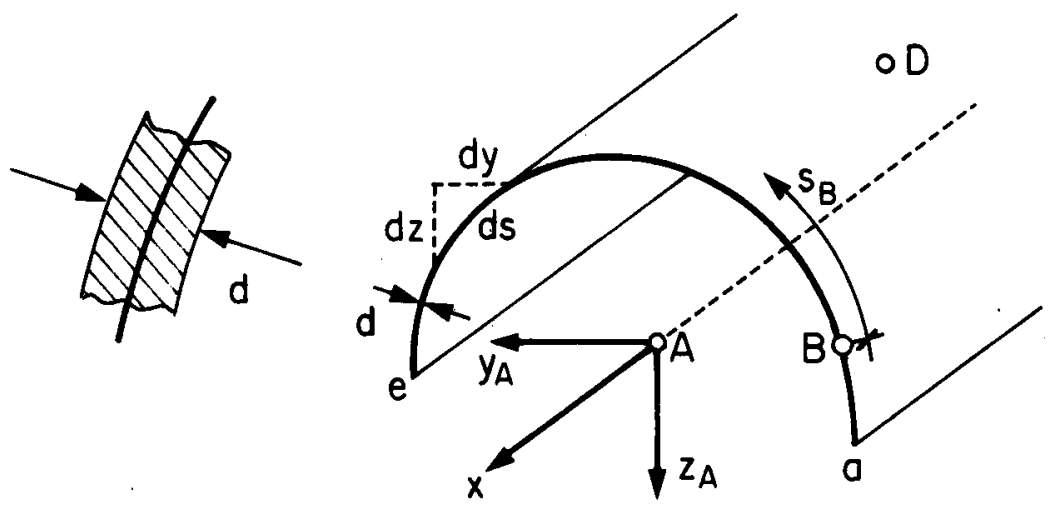

Bild 3

Die Punkte $A$ und $D$ sind beliebige Punkte der Querschnittsebene, der Punkt $B$ ist ein beliebiger Punkt auf der Mittellinie des Querschnittes.

Die Lasten werden wie folgt definiert:

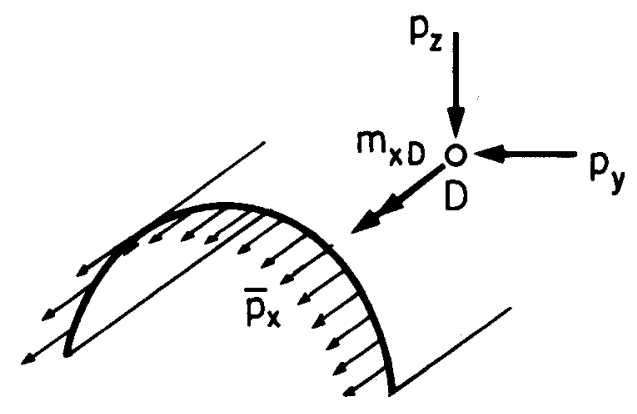

$$
\begin{array}{ll}
p_{y} & {[t / m]} \\
p_{z} & {[t / m]} \\
m_{x D} & {[\mathrm{mt} / \mathrm{m}]} \\
\bar{p}_{x} & {\left[t / \mathrm{m}^{2}\right]}
\end{array}
$$


Weil Querschnittserhaltung vorausgesetzt ist, dürfen die in Querrichtung wirkenden Lasten durch deren Resultierende ersetzt werden. Es sind dies die auf den Punkt $\square$ reduzierten Querbelastungen $p_{y}, p_{z}$ und $m_{x D}$. Die in Stablängsrichtung wirkenden Lasten $\bar{p}_{x}\left[t / \mathrm{m}^{2}\right]$ dürfen hingegen nicht in einer Resultierenden zusammengefasst werden, da sich der Querschnitt in Längsrichtung aus seiner Ebene heraus verwölben kann.

\subsection{Gleichgewichtsbedingungen}

Dank der vorausgesetzten Querschnittserhaltung kann ein Teil der Gleichgewichtsbedingungen wie bei einem stab mit Vollquerschnitt erhalten werden.

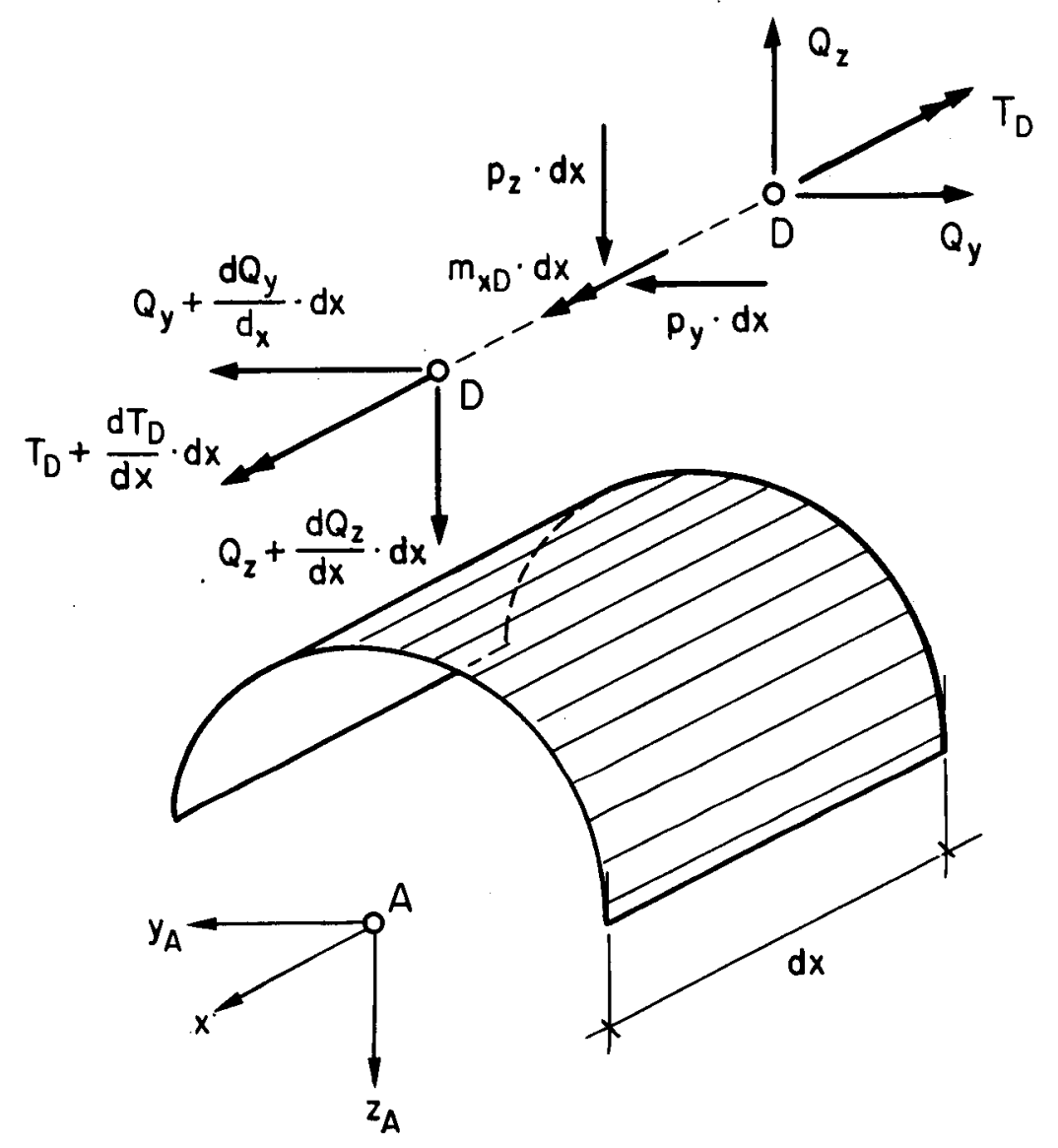


Es sind dies die bekannten Zusammenhänge zwischen den Querkräften $Q_{y}$, $Q_{z}$, dem Torsionsmoment $T_{D}$ und den Querbelastungen.

$$
\begin{aligned}
& \frac{d Q_{y}}{d x}+p_{y}=0 \\
& \frac{d Q_{z}}{d x}+p_{z}=0 \\
& \frac{d T}{d x}+m_{x D}=0
\end{aligned}
$$

Die übrigen Gleichgewichtsbedingungen für ein Stabelement ergeben sich aus der Betrachtung des Schubflusses $S\left(S=\tau_{x s} \cdot d\right)$.

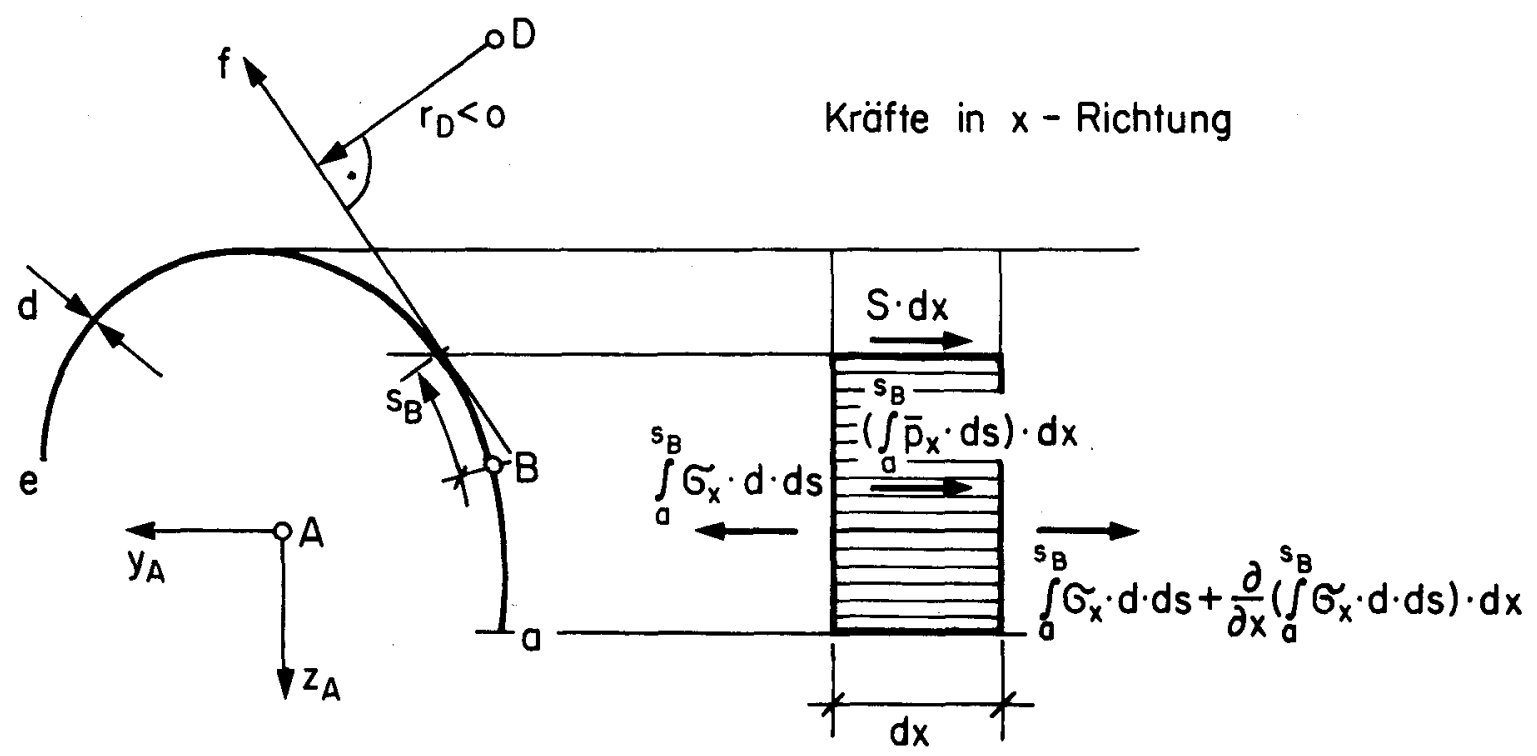

Bild 6

Entsprechend dem rechtsdrehenden Koordinatensystem ist der Abstand $\Gamma_{D}$ positiv, wenn der Punkt $D$ links von der Tangente $f$ liegt (Blickrichtung: Positive s-Koordinate).

Die Gleichgewichtsbedingung in Längsrichtung liefert folgenden Ausdruck für den Schubfluss 5 :

$$
\begin{aligned}
s \cdot d x & =-\frac{\partial}{\partial x}\left(\int_{a}^{s_{B}} \sigma_{x} \cdot d \cdot d s\right) \cdot d x-\left(\int_{a}^{s_{B}} \bar{p}_{x} \cdot d s\right) \cdot d x \\
s & =-\frac{\partial}{\partial x} \int_{a}^{s_{B}} \sigma_{x} \cdot d \cdot d s-\int_{a}^{s_{B}} \bar{p}_{x} \cdot d s
\end{aligned}
$$


Aus der Randbedingung, dass der Schubfluss am Querschnittsende e verschiwinden muss, bekommt man die Beziehung:

$$
a=\frac{d}{d x} \int_{a}^{e} \sigma_{x} \cdot d \cdot d s+\int_{a}^{e} \bar{p}_{x} \cdot d s
$$

Die partiellen Ableitungen $\frac{\partial}{\partial x}$ gehen in gewöhnliche Ableitungen $\frac{d}{d x}$ über, weil der nachfolgende Ausdruck wegen der Integration über die festen Grenzen a-e nur noch eine Funktion der Variablen $x$ ist.

Die im Querschnitt vorhandenen Schubspannungen $\tau_{x s}$ bzw. der entsprechende Schubfluss $S$ müssen zu den Schnittkräften $Q_{y}, Q_{z}$ und $T_{D}$ statisch äquivalent sein. Es gilt:

$$
\begin{aligned}
Q_{y} & =\int_{a}^{e} s \cdot d y \\
Q_{z} & =\int_{a}^{e} s \cdot d z \\
T_{D} & =T_{w D}=\int_{a}^{e} s \cdot r_{D} \cdot d s
\end{aligned}
$$

Die Beziehung $T_{D}=T_{W D}$ besagt, dass die Plattenwirkung und damit die St. Venant'sche Torsion vernachlässigt werden.

Bei Berücksichtigung der St. Venant'schen Torsion würde sich das Torsionsmoment $T_{D}$ aus einem Wölb- und einem St. Venant'schen Anteil zusammensetzen:

$$
\begin{aligned}
& T_{D}=T_{W D}+T_{S} \\
& T_{W D} \text { : Auf den Punkt } D \text { bezogenes Wölbtorsionsmoment. } \\
& T_{S} \text { : St. Venant'sches Torsionsmoment. }
\end{aligned}
$$


Als nächstes wird der Ausdruck (2) in die Gleichungen (4) eingesetzt:

$$
\begin{aligned}
& Q_{y}=\int_{a}^{e} S \cdot d y= \\
& =-\frac{d}{d x} \int_{a}^{e} d y \int_{a}^{s_{B}} \sigma_{x} \cdot d \cdot d s \quad-\int_{a}^{e} d y \int_{a}^{s_{B}} \bar{p}_{x} \cdot d s= \\
& =-\frac{d}{d x} \int_{a}^{e} d y \int_{a}^{e} \sigma_{x} \cdot d \cdot d s-\int_{a}^{e} d y \int_{a}^{e} \bar{p}_{x} \cdot d s+ \\
& +\frac{d}{d x} \int_{a}^{e} \sigma_{x} \cdot d \cdot d s \int_{y_{A}(a)}^{y_{A}} d y+\int_{a}^{e} \bar{p}_{x} \cdot d s \int_{y_{A}(a)}^{y_{A}} d y= \\
& =+\frac{d}{d x} \int_{a}^{e} \sigma_{x} \cdot y_{A} \cdot d \cdot d s+\int_{a}^{e} \bar{p}_{x} \cdot y_{A} \cdot d s \quad- \\
& -\left[y_{A}(a)+\int_{a}^{e} d y\right] \cdot\left[\frac{d}{d x} \int_{a}^{e} \sigma_{x} \cdot d \cdot d s+\int_{a}^{e} \bar{p}_{x} \cdot d s\right] \\
& =0 \\
& T_{D}=T_{w D}=\int_{a}^{e} s \cdot r_{D} \cdot d s= \\
& =-\frac{d}{d x} \int_{a}^{e} r_{D} \cdot d s \int_{a}^{s_{B}} \sigma_{x} \cdot d \cdot d s \quad-\int_{a}^{e} r_{D} \cdot d s \int_{a}^{s_{B}} \bar{p}_{X} \cdot d s= \\
& =-\frac{d}{d x} \int_{a}^{e} r_{D} \cdot d s \int_{a}^{e} \sigma_{x} \cdot d \cdot d s-\int_{a}^{e} r_{D} \cdot d s \int_{a}^{e} \bar{p}_{x} \cdot d s+ \\
& +\frac{d}{d x} \int_{a}^{e} \sigma_{x} \cdot d \cdot d s \int_{s_{B}(a)}^{s_{B}} r_{D} \cdot d s+\int_{a}^{e} \bar{p}_{x} \cdot d s \int_{s_{B}(a)}^{s_{B}} r \cdot d s= \\
& =+\frac{d}{d x} \int_{a}^{e} \sigma_{X} \cdot\left(\int_{0}^{s_{B}} r_{D} \cdot d s\right) \cdot d \cdot d s+\int_{a}^{e} \bar{p}_{X} \cdot\left(\int_{0}^{s_{B}} r_{D} \cdot d s\right) \cdot d s+ \\
& +\left[\int_{s_{B}(a)}^{0} r_{D} \cdot d s-\int_{a}^{e} r_{D} \cdot d s\right] \cdot\left[\frac{d}{d x} \int_{a}^{e} \sigma_{X} \cdot d \cdot d s+\int_{a}^{e} \bar{p}_{X} \cdot d s\right] \\
& =0
\end{aligned}
$$


$\mathrm{s}_{\mathrm{B}}$

Mit der sektoriellen Koordinate $\omega_{D B}=\int r_{D} \cdot d s$, einer geometrischen Grösse, lassen sich die erhaltenen Gleichungen wie folgt anschreiben:

$$
\begin{aligned}
& Q_{y}=\frac{d}{d x} \int_{a}^{e} \sigma_{x} \cdot y_{A} \cdot d \cdot d s+\int_{a}^{e} \bar{p}_{x} \cdot y_{A} \cdot d s \\
& Q_{z}=\frac{d}{d x} \int_{a}^{e} \sigma_{x} \cdot z_{A} \cdot d \cdot d s+\int_{a}^{e} \bar{p}_{x} \cdot z_{A} \cdot d s \\
& T_{w D}=\frac{d}{d x} \int_{a}^{e} \sigma_{x} \cdot \omega_{D B} \cdot d \cdot d s+\int_{a}^{e} \bar{p}_{x} \cdot \omega_{D B} \cdot d s
\end{aligned}
$$

Es sei hier festgehalten, dass die sektorielle koordinate ${ }^{\omega_{D B}}$ um den Punkt $\mathrm{D}$ "dreht" und so normiert ist, dass sie im Punkt B verschwindet.

Die Einführung weiterer Abkürzungen

$$
\begin{aligned}
N & =\int_{a}^{e} \sigma_{x} \cdot d \cdot d s \\
M_{y A} & =\int_{a}^{e} \sigma_{x} \cdot y_{A} \cdot d \cdot d s \\
M_{z A} & =\int_{a}^{e} \sigma_{x} \cdot z_{A} \cdot d \cdot d s \\
M_{\omega D B} & =\int_{a}^{e} \sigma_{x} \cdot \omega_{D B} \cdot d \cdot d s \\
P_{x} & =\int_{a}^{e} \bar{p}_{x} \cdot d s \\
m_{y A} & =\int_{a}^{e} \bar{p}_{x} \cdot y_{A} \cdot d s \\
m_{z A} & =\int_{a}^{e} \bar{p}_{x} \cdot z_{A} \cdot d s \\
m_{\omega D B} & =\int_{a}^{e} \bar{p}_{x} \cdot \omega D B \cdot d s
\end{aligned}
$$

ermöglicht eine zweckmässige und übersichtliche Darstellung der Gleichungen (3) und (6). 


$$
\begin{aligned}
& \frac{d N}{d x}+P_{x}=0 \\
& Q_{y}=\frac{d M_{y A}}{d x}+m_{y A} \\
& Q_{z}=\frac{d M_{z A}}{d x}+m_{z A} \\
& T_{D}=T_{W D}=\frac{d M_{\omega D B}}{d x}+m_{\omega D B}
\end{aligned}
$$

Die physikalische Bedeutung der in (7) eingeführten Grössen ist evident.

$$
\begin{array}{ll}
N & \text { Normalkraft } \\
M_{y A}, M_{z A}: & A u f \text { die Koordinatenaxen } z_{A}=0 \text { und } \\
& y_{A}=0 \text { bezogene Biegemomente. } \\
M_{\omega D B} \quad \text { Um den Punkt } D \text { drehendes und auf den } \\
& \text { Punkt B normiertes Wölbmoment. }
\end{array}
$$

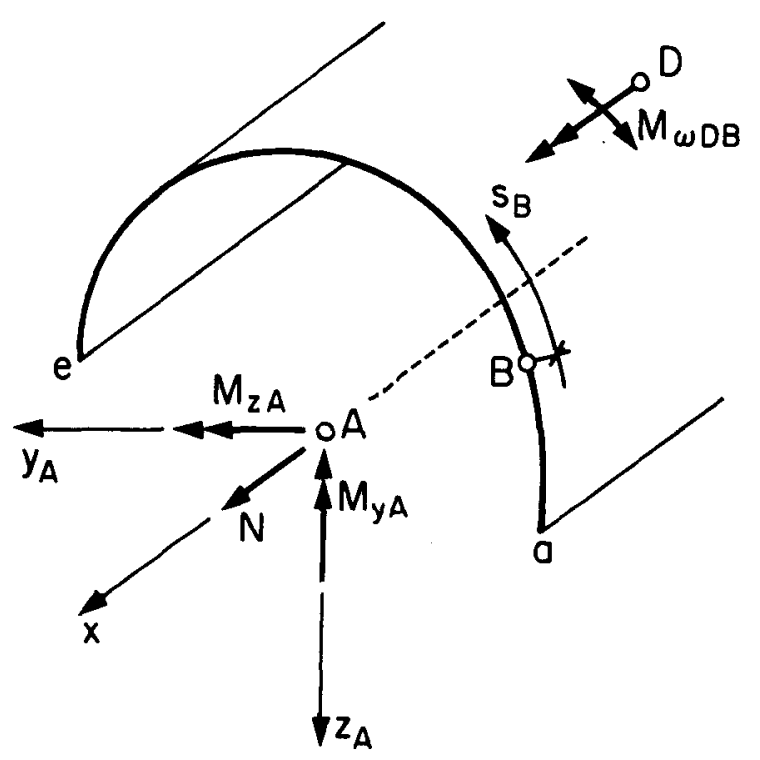


Betrachtet man die in ( 8 ) eingeführten Abkürzungen,

$$
\begin{aligned}
& p_{x}=\int_{a}^{e} \bar{p}_{x} \cdot d s \\
& m_{y A}=\int_{a}^{e} \bar{p}_{x} \cdot y_{A} \cdot d s=p_{x} \cdot \frac{\int_{\int_{a}}^{e} \bar{p}_{x} \cdot y_{A} \cdot d s}{\int_{x} \cdot d s}=p_{x} \cdot y_{A}\left(p_{X}\right) \\
& m_{z A}=\int_{a}^{e} \bar{p}_{X} \cdot z_{A} \cdot d s=p_{x} \cdot z_{A}\left(p_{X}\right) \\
& m_{\omega D B}=\int_{a}^{e} \bar{p}_{x} \cdot \omega_{D B} \cdot d s
\end{aligned}
$$

so erkennt man, dass es im Unterschied zum vollen Stab nicht mehr mäglich ist, die in Richtung der Stabaxe wirkenden Lasten $\bar{p}_{x}$ durch deren Resultierende $p_{x}$ im Punkt $\left[y_{A}\left(p_{x}\right), z_{A}\left(p_{x}\right)\right]$ zu ersetzen. Verantwortlich für diese Tatsache ist die Lastgrösse $\mathrm{m}_{\omega D B}$.

Im Gegensatz dazu dürfen die Querbelastungen durch ihre Resultierenden ersetzt werden, da bei dieser Theorie Querschnittserhaltung vorausgesetzt wird.

Werden nun die letzten drei Gleichungen aus (9) nach $x$ abgeleitet und die Beziehungen aus (1) in diesen Gleichungen eingesetzt, dann erhält man:

$$
\begin{aligned}
& \frac{d^{2} M_{y A}}{d x^{2}}+p_{y}+\frac{d m_{y A}}{d x}=0 \\
& \frac{d^{2} M_{z A}}{d x^{2}}+p_{z}+\frac{d m_{z A}}{d x}=0 \\
& \frac{d^{2} M_{\omega D B}}{d x^{2}}+m_{x D}+\frac{d m_{\omega D B}}{d x}=0
\end{aligned}
$$

Das vollständige Differentialgleichungssystem, welches die Schnittkräfte mit den Lasten verknüpft, kann schliesslich aus den Beziehungen (1), (9) und (10) zusammengestellt werden. 


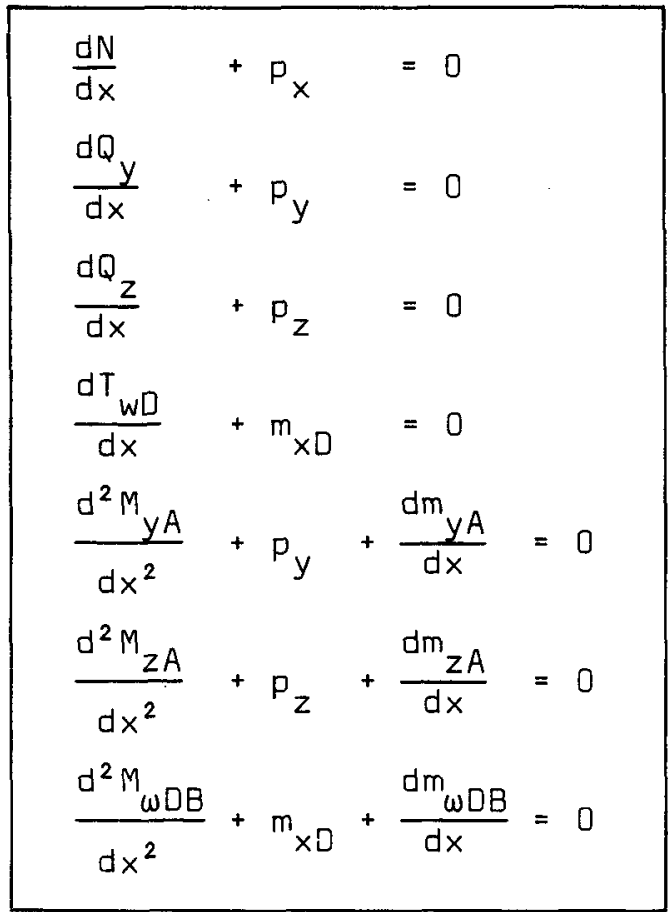

És sei noch einmal daran erinnert, dass die Koordinaten $y_{A}$ und $z_{A}$, die Querschnittskoordinate $s_{B}$ und der Punkt $D$ ganz beliebig gewählt werden können.

Das Gleichungssystem (11) besteht aus sieben linearen, von einander unabhängigen Differentialgleichungen mit konstanten Koeffizienten. Jede Gleichung enthält eine unbekannte Schnittkraft. An statisch bestimmten Systemen, bei denen die Randbedingungen für die Schnittkräfte bekanntlich gegeben sind, können die Schnittkräfte allein aus diesen Gleichungen bestimmt werden.

Ferner ist aus der Zusammenstellung (11) folgende formelle Analogie zwischen Wölbtorsion und Balkenbiegung zu erkennen:

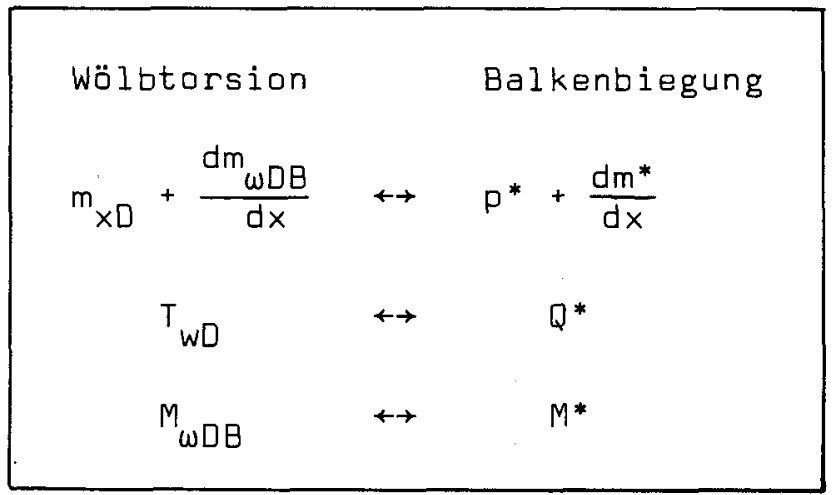

Diese Analogie ermöglicht eine einfache und zudem von der Balkenbiegung her bekannte Bestimmung der Wölbtorsionsschnittkräfte $T_{W D}$ und $M_{\omega D B}$ ' was an einem einfachen Beispiel gezeigt werden soll. 


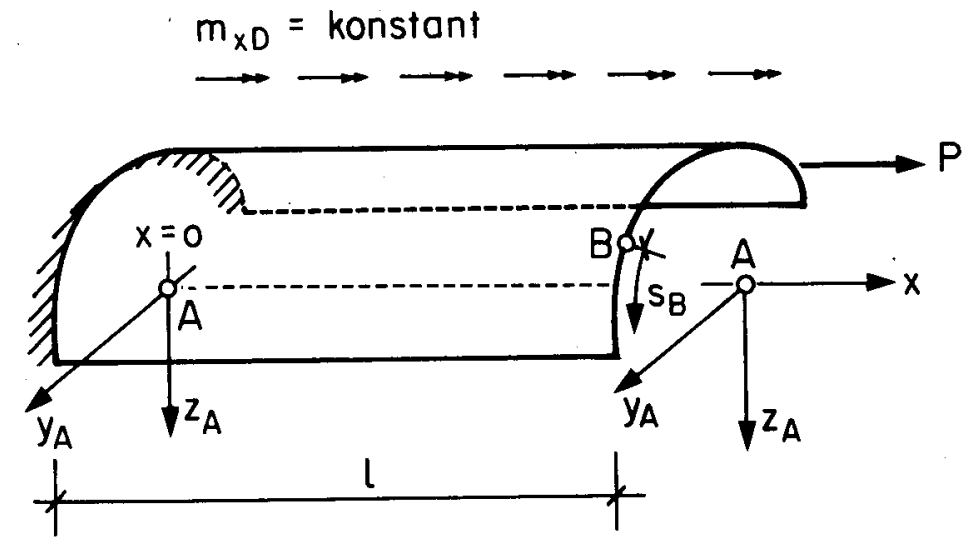

Bestimmung der Schnittkräfte:

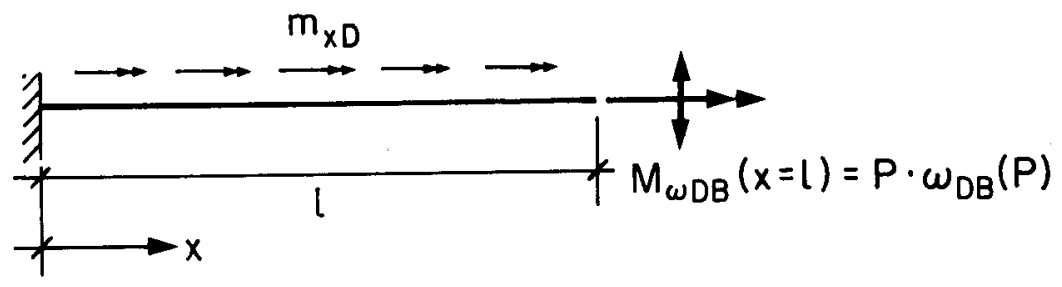

Analogiebalken:
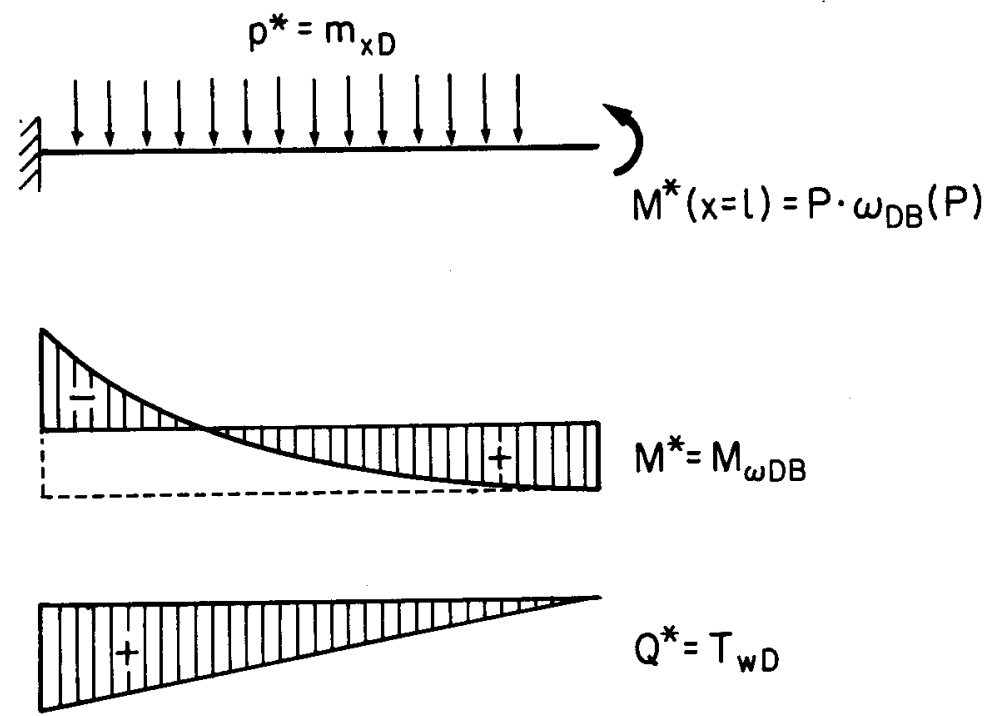
Formelmässig ergeben sich die dargestellten Schnittkräfte zu:

$$
\begin{aligned}
& M_{\omega D B}=M^{*}=P \cdot \omega_{D B}(P)-m_{x D} \cdot \frac{(1-x)^{2}}{2} \\
& T_{W D}=Q^{*}=m_{x D} \cdot(1-x)
\end{aligned}
$$

Nun soll zum Vergleich mit (11), wo nur die wölbtorsion vorkommt, die St. Venant'sche Torsion in den Gleichgewichtsbedingungen mitgenommen werden. In diesem Falle ist die Beziehung (5) in die letzten Gleichungen aus (1) und (9) einzusetzen. Die Gleichgewichtsgleichungen für die gemischte Torsion (Wölb- und St. Venant'sche Torsion) lauten dann:

$$
\begin{aligned}
& \frac{d T_{\omega D}}{d x}+\frac{d T_{s}}{d x}+m_{x D}=0 \\
& \frac{d^{2} M_{\omega D B}}{d x^{2}}+\frac{d T_{s}}{d x}+m_{x D}+\frac{d m_{\omega D B}}{d x}=0
\end{aligned}
$$

Bei gemischter Torsion bekommt man zusätzlich noch eine unbekannte Schnittkraft, nämlich das St. Venant'sche Torsionsmoment $T_{s}$, in die Gleichgewichtsbedingungen. Da jedoch die Anzahl der Gleichungen nicht ändert, ist es selbst bei einem statisch bestimmten Tragwerk narmalerweise nicht möglich, die Schnittkräfte $M_{\omega D \theta}$ ' $T_{W D}$ und $T_{s}$ aus den Gleichgewichtsbedingungen allein zu bestimmen. Demnach stellt das St. Venant'sche Torsionsmoment bei Stäben mit dünnwandigen offenen Querschnitten eine statisch überzählige Grösse dar, welche zur Erhaltung des Gleichgewichts nicht nötig ist. Ausnahmen gibt es in jenen Fällen, wo durch

die Querschnittsform

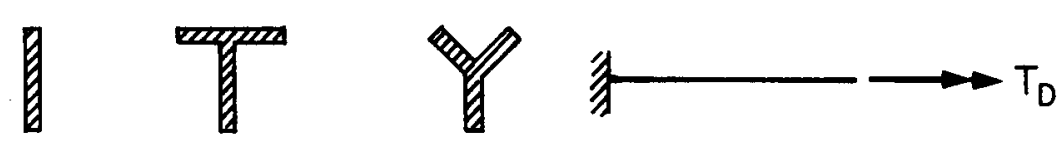

oder die Lagerung

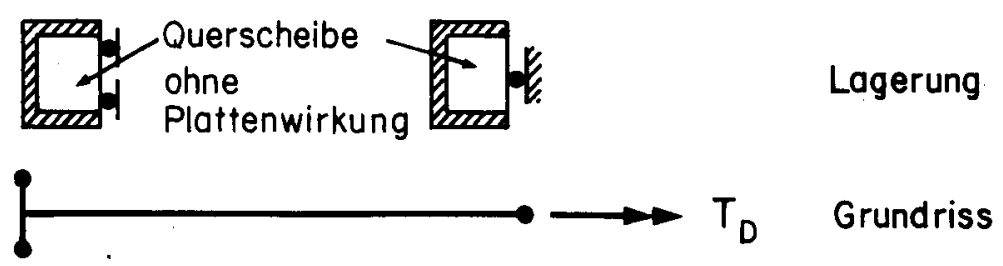


ein Aufbau der Wölbtorsionsschnittkräfte (Scheibenwirkung) verunmöglicht wird. Von derartigen Tragwerken wird im folgenden abgesehen, da die St.Venant'sche Torsion wegen der vorausgesetzten Vernachlässigung der Plattenwirkung nicht berücksichtigt wird.

Neben den Schnittkräften interessieren vor allem die statischen Zusammenhänge zwischen den Schnittkräften und den Spannungen. Die betreffenden Gleichungen sind bereits in (4) und (7) formuliert und brauchen nur noch zusammengestellt zu werden.

$$
\begin{aligned}
N & =\int_{a}^{e} \sigma_{x} \cdot d \cdot d s \\
Q_{y} & =\int_{a}^{e} s \cdot d y \\
Q_{z} & =\int_{a}^{e} s \cdot d z \\
T_{w D} & =\int_{a}^{e} s \cdot r_{D} \cdot d s \\
M_{y A} & =\int_{a}^{e} \sigma_{x} \cdot y_{A} \cdot d \cdot d s \\
M_{z A} & =\int_{a}^{e} \sigma_{x} \cdot z_{A} \cdot d \cdot d s \\
M_{\omega D B} & =\int_{a}^{e} \sigma_{x} \cdot \omega_{D B} \cdot d \cdot d s
\end{aligned}
$$

Mit den geometrischen Beziehungen

$$
\begin{aligned}
& d y=d s \cdot \cos \delta \\
& d z=d s \cdot \sin \delta
\end{aligned}
$$

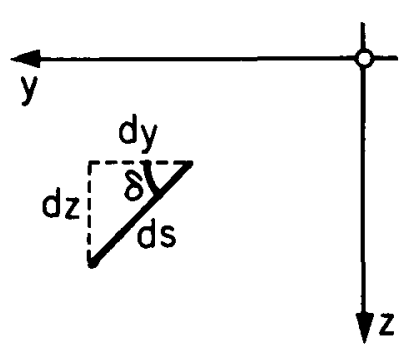


lassen sich diese Gleichgewichtsbedingungen in einer Form darstellen, wie sie für die spätere Berechnung der plastischen Querschnittswiderstände zweckmässig ist.

$\begin{aligned} N & =\int_{a}^{e} \sigma_{x} \cdot d \cdot d s \\ Q_{y} & =\int_{a}^{e} s \cdot \cos \delta \cdot d s \\ Q_{z} & =\int_{a}^{e} s \cdot s i n \delta \cdot d s \\ T_{w D} & =\int_{a}^{e} s \cdot r_{D} \cdot d s \\ M_{y A} & =\int_{a}^{e} \sigma_{x} \cdot y_{A} \cdot d \cdot d s \\ M_{z A} & =\int_{a}^{e} \sigma_{x} \cdot z_{A} \cdot d \cdot d s \\ M_{\omega D B} & =\int_{a}^{e} \sigma_{x} \cdot \omega_{D B} \cdot d \cdot d s\end{aligned}$

\subsection{Kinematische Bedingungen}

In diesem Abschnitt wird der Verschiebungszustand eines Stabelementes untersucht. Zur Einführung dient die kinematische Betrachtung eines Balkens, dessen Verschiebungszustand durch die Verschiebungen $u$ und $w$ gegeben ist.

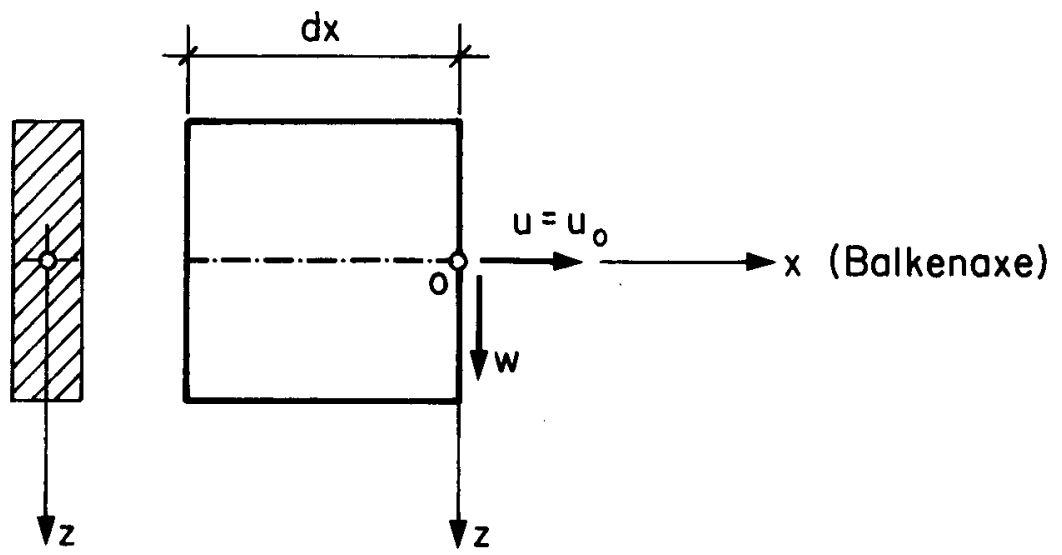


Für die Verschiebungen $u$ und $w$ werden folgende Ansätze gemacht:

$$
\begin{aligned}
& u=u_{0}(x)-\frac{d w_{0}(x)}{d x} \cdot z \\
& w=w_{0}(x)+\int_{x} \gamma_{0}(x) \cdot d x
\end{aligned}
$$

In diesen Ansätzen ist die in der Balkenstatik übliche Voraussetzung ebenbleibender Querschnitte enthalten. Die Verschiebung $w_{0}$ bezeichnet nicht die gesamte Durchbiegung in Richtung der z-Axe, sondern lediglich den schiebungsfreien Anteil derselben. Aus den Verschiebungen u und w erhält man die gesuchten Dehnungen $\varepsilon_{x}$ und $\varepsilon_{z}$ sowie die Schiebung $\gamma_{x z}$ durch Ableitung.

$$
\begin{aligned}
& \varepsilon_{x}=\frac{\partial u}{\partial x}=\frac{d u_{0}}{d x}-\frac{d^{2} w_{0}}{d x^{2}} \cdot z \\
& \varepsilon_{z}=\frac{\partial w}{\partial z}=0 \\
& \gamma_{x z}=\frac{\partial u}{\partial z}+\frac{\partial w}{\partial x}=\gamma_{0}
\end{aligned}
$$

Wie man sieht, beeinflussen die Verschiebungskomponenten $u_{0}$ und $w_{0}$ nur die Längsdehnungen $\varepsilon_{x}$, während $\gamma_{0}$ die Schiebung des Querschnittes beschreibt.

Für den Fall des dünnwandigen offenen Querschnittes wird analog vorgegangen. Es wird zwischen Verschiebungskomponenten (wie $u_{0}$ und $w_{0}$ ), welche den Dehnungszustand beschreiben, und zwischen sogenannten Schiebungskomponenten (entsprechend $\gamma_{0}$ ), die lediglich die Schiebungen beeinflussen, unterschieden. Da gemäss Voraussetzung Querschnittserhaltung angenommen wird, kann der Verschiebungszustand durch vier Verschiebungskomponenten und drei Schiebungsgrössen dargestellt werden.

Zuerst wird der Einfluss der vier Verschiebungskomponenten untersucht.

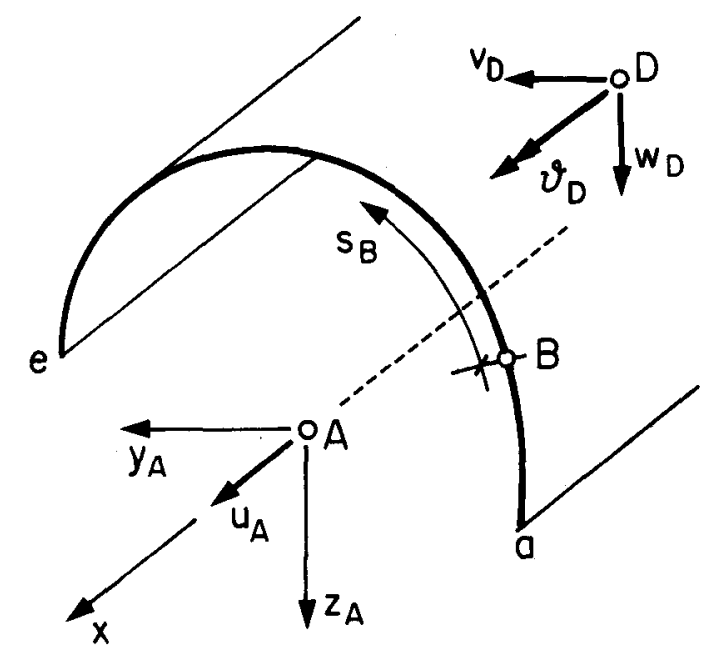


Jede der vier dargestellten Verschiebungskomponenten, bzw. deren Ableitungen nach $x$, bewirkt eine Verschiebung in Stablängsrichtung.

Die Längsverschiebungen infolge $v_{D}$ und $w_{D}$ können aus der nachstehenden $F_{i-}$ gur abgelesen werden.
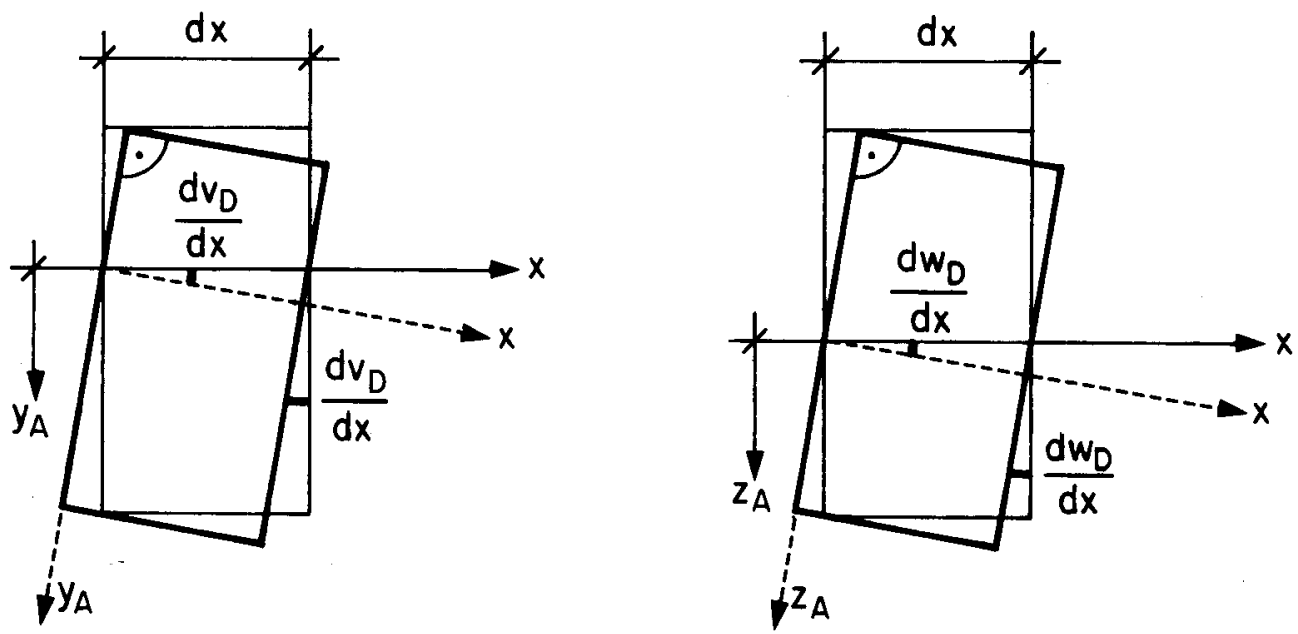

Bild 13

Sie werden wie früher die Biegemomente auf den Punkt A normiert.

$$
\begin{aligned}
& u\left(v_{D}\right)=-\frac{d v_{D}}{d x} \cdot y_{A} \\
& u\left(w_{D}\right)=-\frac{d w_{D}}{d x} \cdot z_{A}
\end{aligned}
$$

Die Bestimmung der Längsverschiebung infolge $\vartheta_{D}$ erfolgt am einfachsten über die Verschiebung der Mittellinie des Querschnittes.

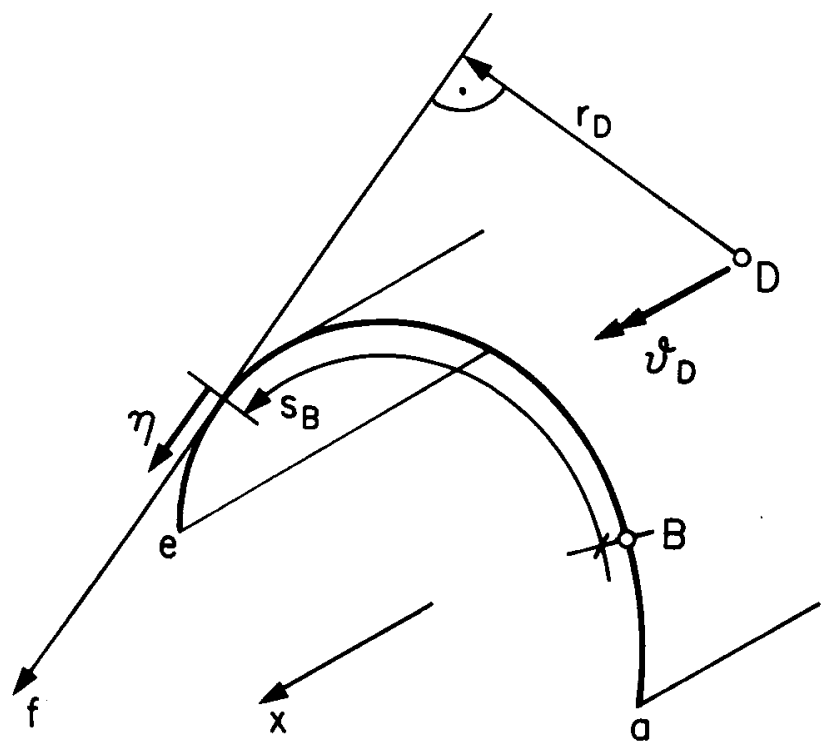


Mit der durch die Drehung $\vartheta_{D}$ bedingten Tangentialverschiebung $n$ der Querschnittsmittellinie bzw. deren Ableitung nach $x$

$$
\begin{aligned}
& n=\vartheta_{D} \cdot r_{D} \\
& \frac{\partial \eta}{\partial x}=\frac{\partial}{\partial x}\left(\vartheta_{D} \cdot r_{D}\right)=\frac{d \vartheta_{D}}{d x} \cdot r_{D}
\end{aligned}
$$

kann folgende Verschiebungsfigur gezeichnet werden:

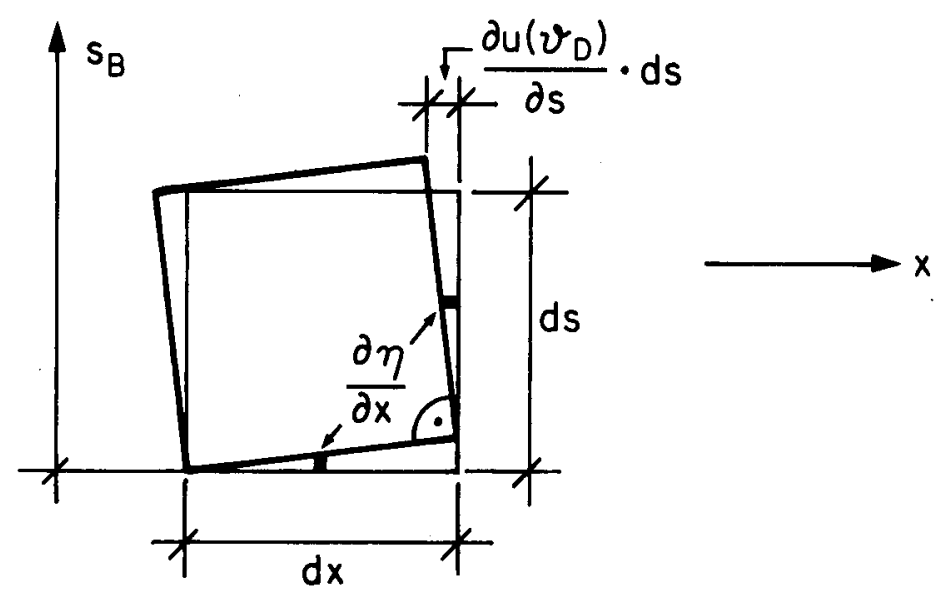

Daraus ergibt sich die Längsverschiebung infolge $\vartheta_{D}$, welche wie früher das Wölbmoment auf den Punkt $B$ normiert wird.

$$
\begin{aligned}
\frac{\partial u\left(\vartheta_{D}\right)}{\partial s} \cdot d s & =-\frac{\partial \eta}{\partial x} \cdot d s=-\frac{d \vartheta_{D}}{d x} \cdot r_{D} \cdot d s \\
u\left(\vartheta_{D}\right) & =-\frac{d \vartheta_{D}}{d x} \cdot \frac{\int_{D} r_{D} \cdot d s}{\omega_{D B}} \\
u\left(\vartheta_{D}\right) & =-\frac{d \vartheta_{D}}{d x} \cdot \omega_{D B}
\end{aligned}
$$

Aus der totalen Längsverschiebung, die man durch Summation der Anteile infolge $u_{A}, v_{D}$, $w_{D}$ und $\vartheta_{D}$ erhält, folgt:

$$
u=u_{A}-\frac{d v_{D}}{d x} \cdot y_{A}-\frac{d w_{D}}{d x} \cdot z_{A}-\frac{d \vartheta_{D}}{d x} \cdot w_{D B}
$$


und die dazugehörige Dehnung $\varepsilon_{x}$ :

$$
\begin{aligned}
& \varepsilon_{x}=\frac{\partial u}{\partial x} \\
& \varepsilon_{x}=\frac{d u_{A}}{d x}-\frac{d^{2} v_{D}}{d x^{2}} \cdot y_{A}-\frac{d^{2} w_{D}}{d x^{2}} \cdot z_{A}-\frac{d^{2} \vartheta_{D}}{d x^{2}} \cdot \omega_{D B}
\end{aligned}
$$

Als nächstes werden die drei Schiebungsgrössen, und zwar je eine Schiebung in $y$ - und $z$-Richtung sowie eine Schiebung um den Punkt $D$, eingeführt.

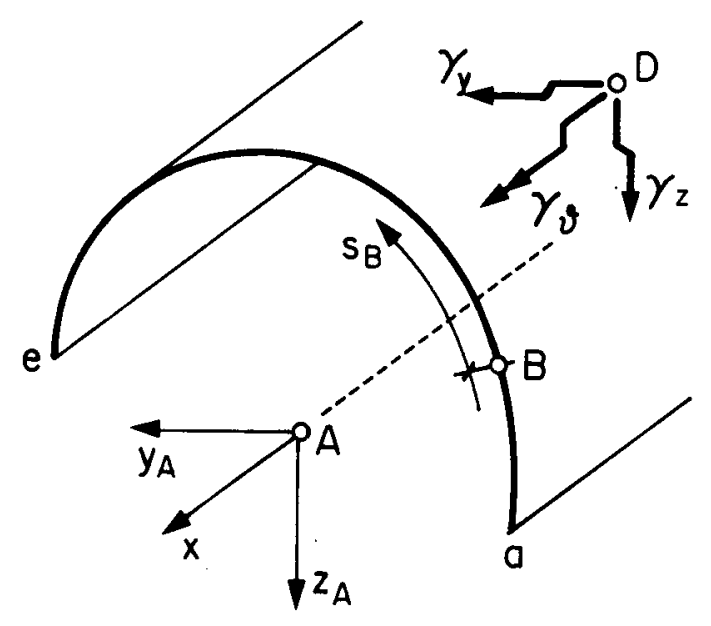

Bild 16

Alle Schiebungen, seien es diejenigen in $y$ - und z-Richtung oder die DrehSchiebung um den Punkt $D$, führen zu folgenden Verformungen.

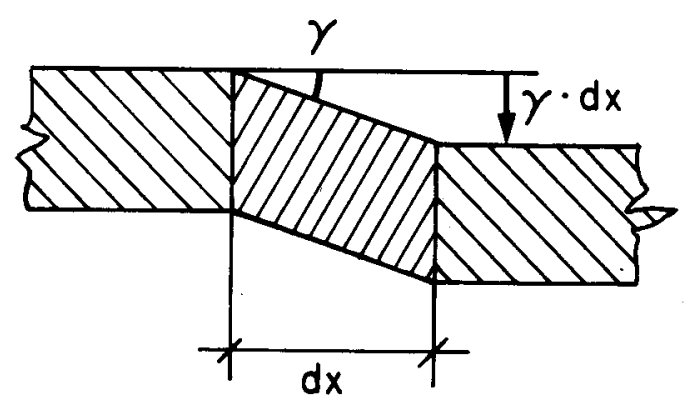


Die Schiebung $\gamma_{x s}$ der Querschnittsmittellinie wird der Einfachheit halber mit $\gamma$ bezeichnet. Den einen Anteil zu dieser Schiebung liefern die Schiebungsgrössen $\gamma_{y}$ und $\gamma_{z}$.
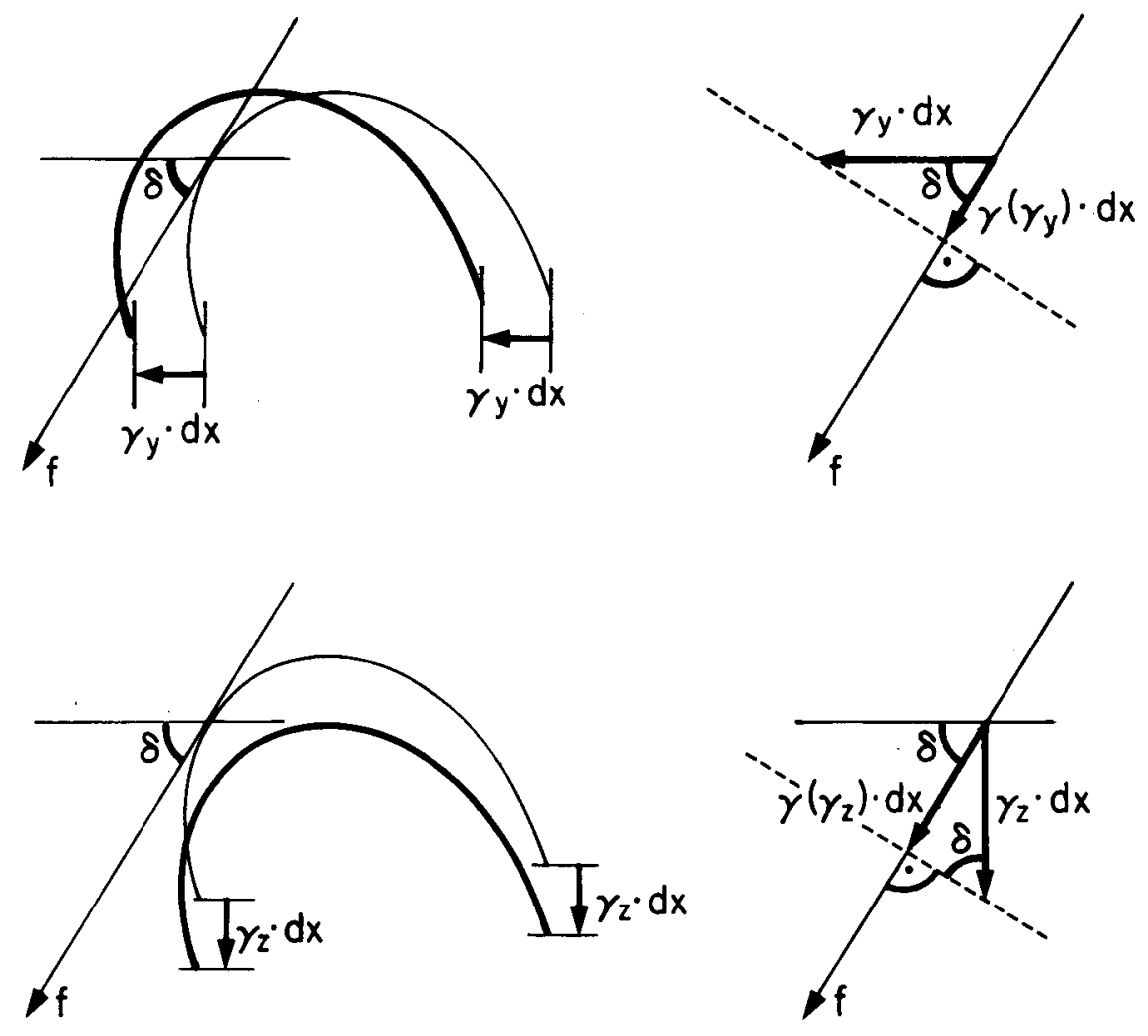

Bild 18

Aus den Detailskizzen dieser Zeichnungen lassen sich die Schiebungen der Querschnittsmittellinie infolge $\gamma_{y}$ und $\gamma_{z}$ ablesen:

$$
\begin{aligned}
& \gamma\left(\gamma_{y}\right)=\gamma_{y} \cdot \cos \delta \\
& \gamma\left(\gamma_{z}\right)=\gamma_{z} \cdot \sin \delta
\end{aligned}
$$


Als nächstes wird die Verschiebungsfigur für die Dreh-Schiebung $\gamma_{\vartheta}$ um den Punkt $D$ bestimmt.

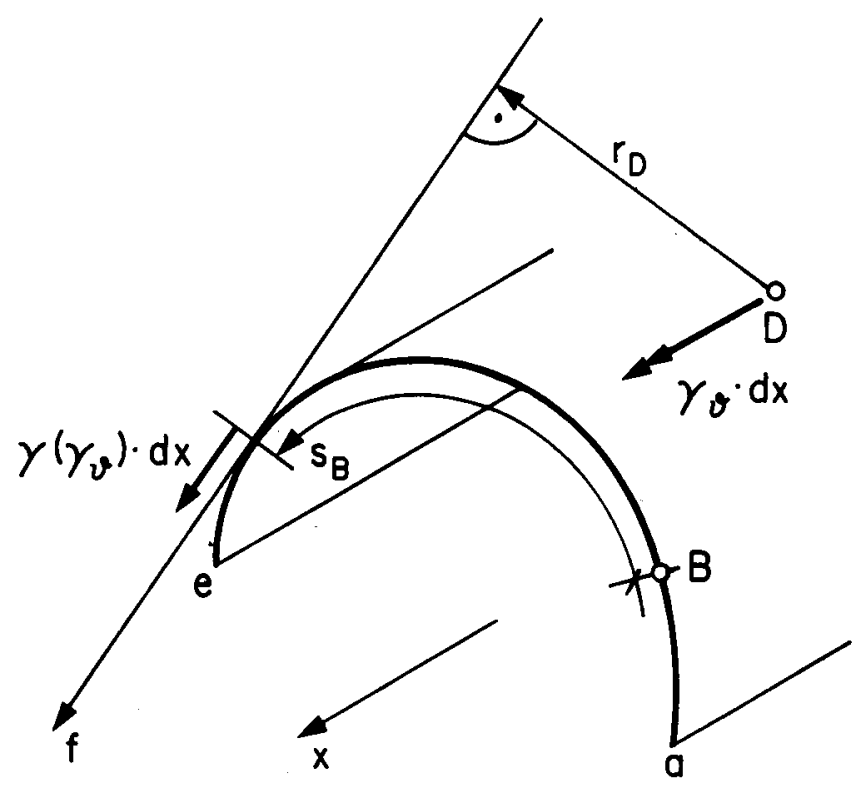

Bild 19

Daraus erhält man die durch $\gamma_{\vartheta}$ bedingte Schiebung der Querschnittsmittellinie.

$$
\gamma\left(\gamma_{\vartheta}\right)=\gamma_{\vartheta} \cdot r_{D}
$$

Die totale Schiebung $\gamma$ der Querschnittsmittellinie kann schliesslich wie folgt angegeben werden.

$$
\gamma=\gamma_{y} \cdot \cos \delta+\gamma_{z} \cdot \sin \delta+\gamma_{\vartheta} \cdot r_{D}
$$


Zum Schluss dieses Abschnittes sei darauf hingewiesen, dass von nun an bei Koordinaten, Lasten, Schnittkräften und Verschiebungen die spezielle Bezeichnung für den jeweiligen Bezugspunkt der Einfachheit halber weggelassen wird.

Zum Beispiel:

$$
\begin{aligned}
& \varepsilon_{x}=\frac{d u}{d x}-\frac{d^{2} v}{d x^{2}} \cdot y-\frac{d^{2} w}{d x^{2}} \cdot z-\frac{d^{2} \vartheta}{d x^{2}} \cdot \omega \\
& M_{w}=\int_{a}^{e} \sigma_{x} \cdot \omega \cdot d \cdot d s \\
& m_{y}=\int_{a}^{e} \bar{p}_{x} \cdot y \cdot d s
\end{aligned}
$$

usw. 
Verzweigte offene Querschnitte können aus einem sogenannten Hauptquerschnitt und aus abstehenden Querschnittsteilen zusammengesetzt werden.

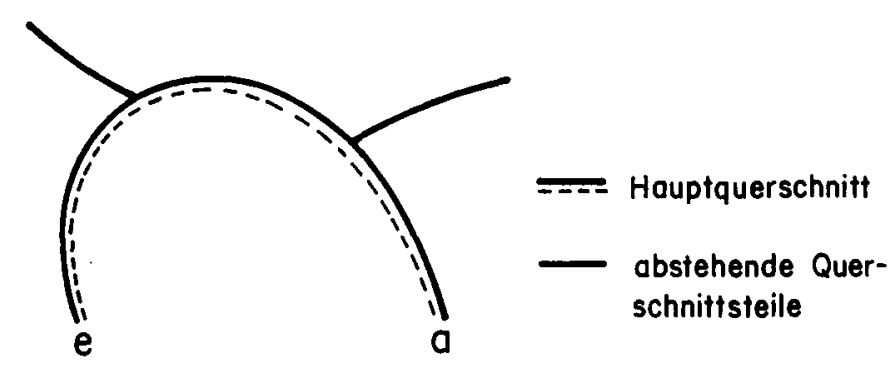

Bild 20

Wenn die Querschnittskoordinaten $s_{a b}$ der abstehenden Teile gegen der Hauptquerschnitt laufen

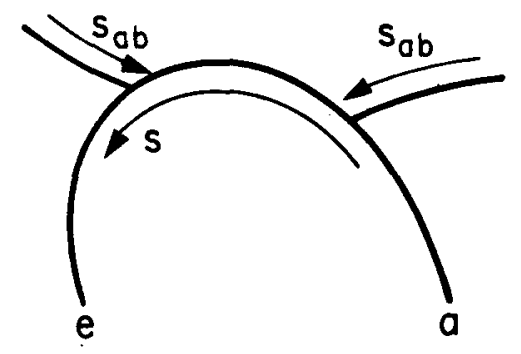

Sab: Querschnittskoordinaten der $a b$ stehenden Teile

S : Querschnittskoordinate des Hauptquerschnittes

Bild 21

und die sektorielle oder Wölbkoordinate $\omega$ in jedem Verzweigungspunkt für den Hauptquerschnitt und den abstehenden Querschnittsteil dieselbe Grösse aufweist, können verzweigte Querschnitte genau gleich wie nichtverzweigte behandelt werden.

Die Berechnung der sektoriellen Koordinaten für die abstehenden Querschnittsteile erfolgt also über die Anfangswerte in den Verzweigungspunkten, welche sich am Hauptquerschnitt allein bestimmen lassen. 


\section{BESTIMMUNG DER SCHNITTKRAEFTE}

\subsection{Elastizitätstheorie}

Die Berechnung von Stäben mit dünnwandigen offenen Querschnitten nach der Elastizitätstheorie ist bekannt und wurde in zahlreichen Publikationen, z.B. [1] bis [4], veröffentlicht.

Hier wird nur soweit auf diese Theorie eingegangen als sie für den Bruchnachweis von Querschnitten aufgrund elastisch bestimmter Schnittkräfte von Bedeutung ist. In diesem Zusammenhang sei erwähnt, dass die Schnittkräfte eines statisch unbestimmten Systems oft nach der Elastizitätstheorie bestimmt werden. Dann wird, ausgehend von diesen Schnittkräften, ein Sicherheitsnachweis gegen Querschnittsversagen oder eine Querschnittsbemessung auf Bruchwiderstand durchgeführt, wie dies zum Beispiel im Stahlbeton- und Spannbetoribau seit einigen Jahren üblich ist.

In der Elastizitätstheorie werden die Spannungen und die Dehnungen bzw. Schiebungen durch das Hooke'sche Gesetz miteinander verknüpft. Es lautet bei vernachlässigter Poisson'scher Zahl:

$$
\begin{aligned}
& \sigma=E \cdot \varepsilon \\
& \tau=G \cdot \gamma \\
& E: \text { Elastizitätsmodul } \\
& G: \text { Schubmodul. }
\end{aligned}
$$

Die Schubverformungen (Schiebungen) infolge Querkräfte und Wälbtorsionsmoment werden in der üblichen Festigkeitslehre vernachlässigt. Demnach sind nach dieser Theorie nur die Schubverformungen aus der St. Venant'schen Torsion verschieden von Null.

Falls die St. Venant'sche Torsion berücksichtigt wird, erhält man aus den betreffenden Schubverformungen den bekannten Zusammenhang zwischen Verdrehung und St. Venant'schem Torsionsmoment,

$$
T_{s}=G K \cdot \frac{d \vartheta}{d x}
$$

wobei GK die St. Venant'sche Torsionssteifigkeit darstellt.

Durch Anwendung des Hooke'schen Gesetzes auf die Beziehung (19) kann man die 
Längsspannungen $\sigma_{x}$ in Funktion der Verschiebungskomponenten angeben.

$$
\sigma_{x}=E \cdot\left(\frac{d u}{d x}-\frac{d^{2} v}{d x^{2}} \cdot y-\frac{d^{2} w}{d x^{2}} \cdot z-\frac{d^{2} \vartheta}{d x^{2}} \cdot \omega\right)
$$

Dieser Ausdruck wird nun in die Gleichungen (7) für die Schnittkräfte $N, M_{y}$, $M_{z}$ und $M_{\omega}$ eingesetzt. Die Ableitungen nach $x$ werden durch Striche angedeutet.

Mit den Abkürzungen

$$
\begin{array}{rlrl}
F & =\int_{a}^{e} d \cdot d s & & \\
I_{y} & =\int_{a}^{e} y^{2} \cdot d \cdot d s & I_{z}=\int_{a}^{e} z^{2} \cdot d \cdot d s & I_{\omega}=\int_{a}^{e} \omega^{2} \cdot d \cdot d s \\
I_{\omega y} & =\int_{a}^{e} \omega \cdot y \cdot d \cdot d s & I_{y z}=\int_{a}^{e} y \cdot z \cdot d \cdot d s & I_{\omega z}=\int_{a}^{e} \omega \cdot z \cdot d \cdot d s \\
s_{y}=\int_{a}^{e} y \cdot d \cdot d s & S_{z}=\int_{a}^{e} z \cdot d \cdot d s & S_{\omega}=\int_{a}^{e} \omega \cdot d \cdot d s
\end{array}
$$

lassen sich die erwähnten Schnittkräfte folgendermassen anschreiben:

$$
\begin{aligned}
& N=E F \cdot u^{\prime}-E S_{y} \cdot v^{\prime \prime}-E S_{z} \cdot w^{\prime \prime}-E S_{\omega} \cdot \vartheta " \\
& M_{y}=E S_{y} \cdot u^{\prime}-E I_{y} \cdot v^{\prime \prime}-E I_{y z} \cdot w^{\prime \prime}-E I_{w y} \cdot \vartheta " \\
& M_{z}=E S_{z} \cdot u^{\prime}-E I_{y z} \cdot v^{\prime \prime}-E I_{z} \cdot w^{\prime \prime}-E I_{\omega z} \cdot \vartheta " \\
& M_{\omega}=E S_{\omega} \cdot u^{\prime}-E I_{w y} \cdot v^{\prime \prime}-E I_{w z} \cdot w^{\prime \prime}-E I_{\omega} \cdot \vartheta "
\end{aligned}
$$

Als nächstes werden diese Beziehungen in die erste, fünfte und sechste Gleichung des Tableaus (11) und, da die St. Venant'sche Torsion noch berücksichtigt wird, in die zweite Gleichung aus (13) eingesetzt. Auf diese Weise erhält man die aus der Elastizitätstheorie bekannten Differentialgleichungen für dünnwandige, gerade und prismatische stäbe. 


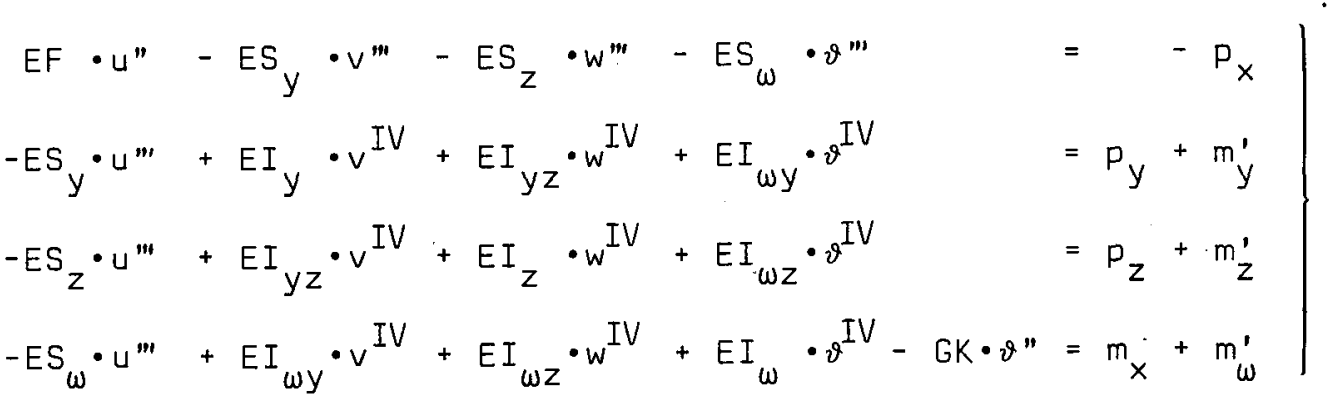

Durch die Wahl eines speziellen Bezugssystems gelingt es, das Differentialgleichungssystem (28) zu entkoppeln.

Wenn die Koordinatenaxen y und $z$ mit den Haupt-Schweraxen des Querschnitts identisch sind, und die sektorielle koordinate (Wälbkoordinate) w auf den Schubmittelpunkt bezogen und so normiert wird, dass das. Integral $\int_{a}^{e} \omega \cdot d \cdot d s$ verschwindet, dann werden $S_{y}=S_{z}=S_{\omega}=I_{y z}=I_{\omega y}=I_{\omega z}=0$.

Damit bekommt man folgende voneinander unabhängige Differentialgleichungen:

$$
\begin{array}{ll}
E F \cdot u " & =-p_{x} \\
E I_{y} \cdot v^{I V} & =p_{y}+m_{y}^{\prime} \\
E I_{z} \cdot w & =p_{z}+m_{z}^{\prime} \\
E I_{w} \cdot \vartheta & I V-G K \cdot \vartheta "=m_{x}+m_{\omega}^{\prime}
\end{array}
$$

Die entkoppelten Gleichungen (29) ermöglichen eine einfache Bestimmung der Verschiebungskomponenten $u, v, w$ und $\vartheta$. Die Schnittkräfte sind Funktionen dieser Verschiebungen. Dank Verwendung des erwähnten Bezugsystem können die Schnittkräfte (27) als einfache Funktionen der Verschiebungskomponenten dargestellt werden. Ausserdem wird die Beziehung (24) für das St. Venant'sche Torsionsmoment übernommen.

$$
\begin{aligned}
& N=E F \cdot u^{\prime} \\
& M_{y}=-E I_{y} \cdot v^{\prime \prime} \\
& M_{z}=-E I_{z} \cdot w^{\prime \prime} \\
& M_{w}=-E I_{\omega} \cdot \vartheta^{\prime \prime} \\
& T_{s}=G K \cdot \vartheta^{\prime}
\end{aligned}
$$


Aus diesen Schnittkräften erhält man schliesslich mit Hilfe der Gleichgewichtsbedingungen (9) die Querkräfte und das Wälbtorsionsmoment.

$$
\begin{aligned}
& Q_{y}=M_{y}^{\prime}+m_{y} \\
& Q_{z}=M_{z}^{\prime}+m_{z} \\
& T_{w}=M_{w}^{\prime}+m_{w}
\end{aligned}
$$

Wird nun ein Querschnitt auf Bruchwiderstand bemessen, wobei von den Schnittkräften (30) und (31) ausgegangen wird, so muss der Bruchwiderstand auf dieselben Koordinaten $y, z$ und $w$, d.h. auf die Haupt-Schweraxen und die normierte, um den Schubmittelpunkt drehende Wölbkoordinate, bezogen werden.

In praktisch vorkommenden Tragwerken mit dünnwandigen offenen stäben ist die St. Venant'sche Torsionssteifigkeit oft vernachlässigbar. In diesem Zusammenhang sei auch erwähnt, dass bei Stahlbeton- und Spannbetonträgern die Torsionssteifigkeit GK durch das Auftreten von Rissen viel stärker reduziert wird als die Biegesteifigkeiten $E I_{y}$ und $E I_{z}$. Diese in Versuchen festgestelite Tatsache führt dazu, dass durch das Reissen des Betons auch die Wölbsteifigkeit $E_{\omega}$ viel langsamer abnimmt als die Torsionssteifigkeit GK. Damit verliert bei offenen Stahlbeton- und Spannbetonträgern die St.Venant'sche Torsion im gerissenen Zustand gegenüber der Wölbtorsion noch mehr an Bedeutung.

Ist die St. Venant'sche Torsionssteifigkeit vernachlässigbar, so vereinfacht sich das Gleichungssystem (29) zu:

$$
\begin{aligned}
& E F \cdot u^{\prime \prime}=-p_{x} \\
& E I_{y} \cdot v^{I V}=p_{y}+m_{y}^{\prime} \\
& E I_{z} \cdot w^{I V}=p_{z}+m_{z}^{\prime} \\
& E I_{w} \cdot v^{I V}=m_{x}+m_{w}^{\prime}
\end{aligned}
$$


Mit den Beziehungen (30) und (31) kann man daraus folgende formelle Analogie zwischen Wölbtorsion und Balkenbiegung entnehmen:

Wälbtorsion

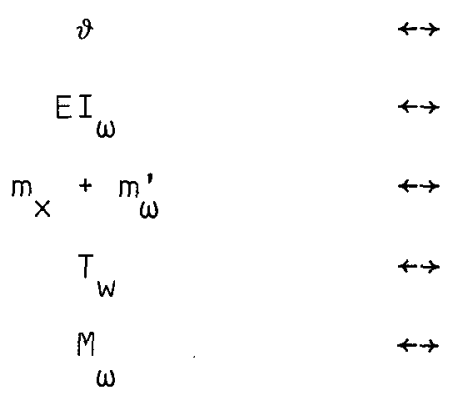

Balkenbiegung

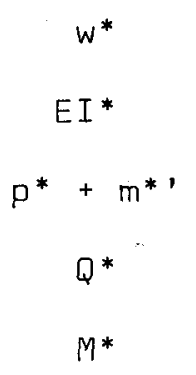

Im Falle reiner Wölbtorsion wird daher die statische Berechnung besonders einfach. Wegen der bestehenden Analogie kann man die bekannten Berechnungsverfahren für Biegeträger auch zur Lösung dieses Torsionsproblems verwenden.

Die Ermittlung der Wölbtorsionsschnittkräfte nach der Biegeanalogie soll an folgendem einfachen Beispiel gezeigt werden. 


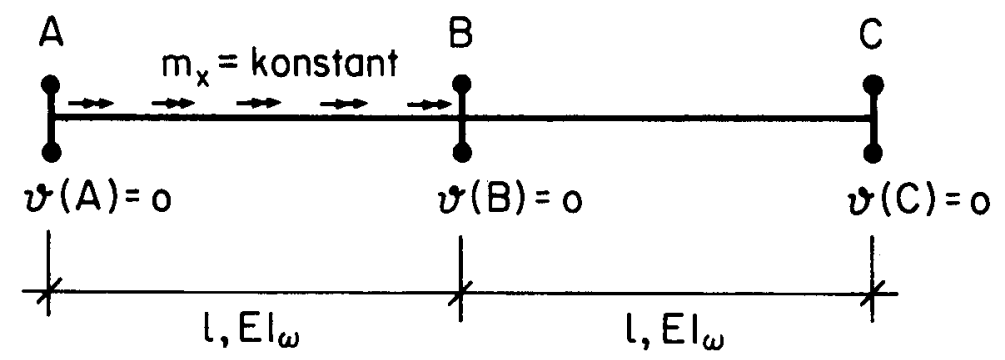

Analogiebalken:
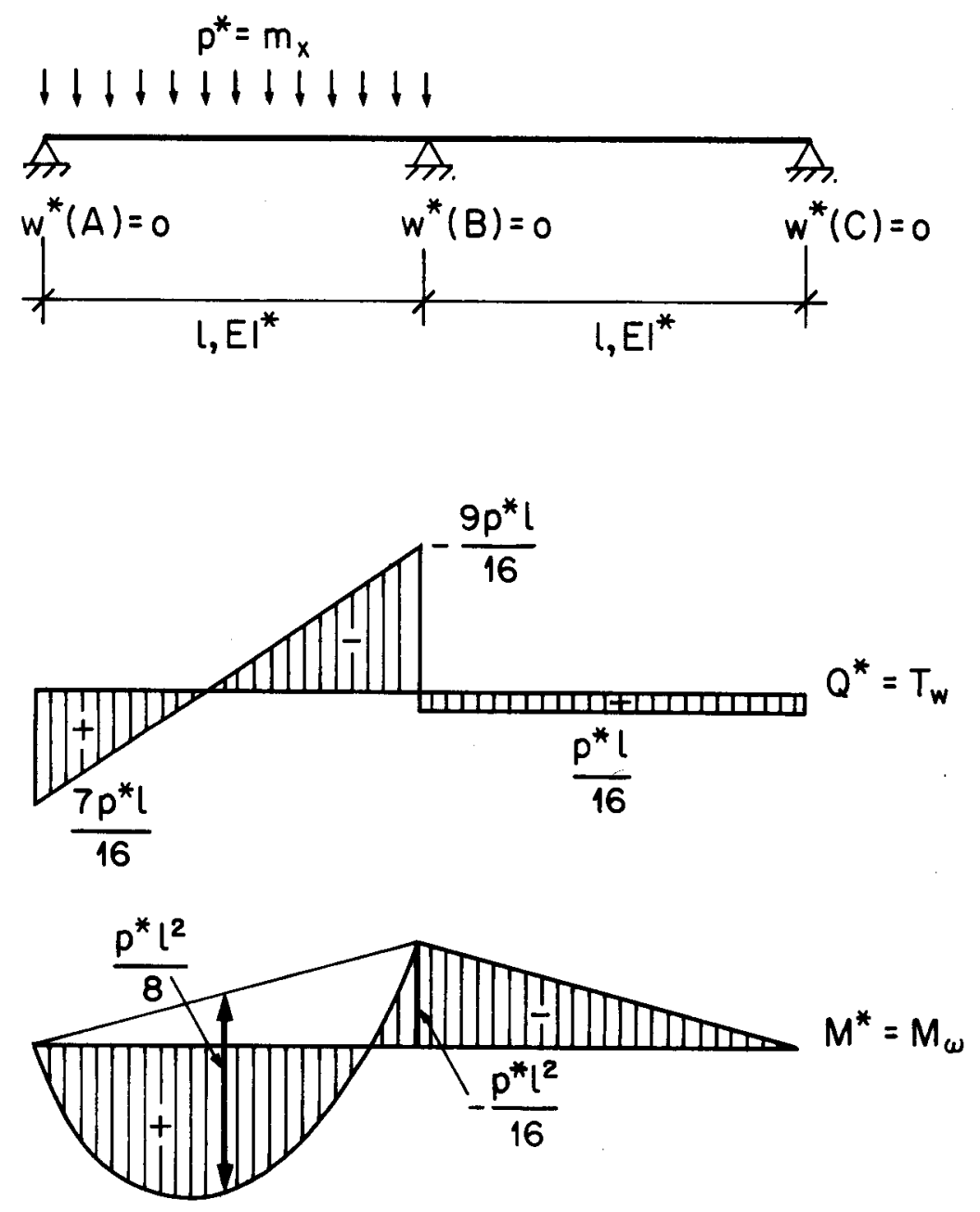


\subsection{Plastizitätstheorie}

Für Stabtragwerke muss eine Lösung nach der Plastizitätstheorie folgende Bedingungen erfüllen:

1. Gleichgewicht

2. Plastizitätsbedingung, d.h. in keinem Querschnitt darf die Beanspruchung den plastischen Querschnittswiderstand überschreiten.

3. Mechanismusbedingung, d.h. es müssen eine gewisse Anzahl Querschnitte plastifiziert sein, so dass sich im Tragwerk ein Mechanismus ausbilden kann.

Wenn hier und im folgenden von Querschnitten die Rede ist, so ist nicht ein Querschnitt im streng geometrischen Sinne gemeint, sondern ein Bereich, der auch in Längsrichtung eine gewisse Ausdehnung hat.

Bei statisch bestimmten Systemen ist die Mechanismusbedingung erfüllt, wenn die Plastifizierung, d.h. der Bruchwiderstand eines Querschnittes erreicht wird. Die Schnittkraftverteilung ist dann nur von den Gleichgewichtsbedingungen abhängig.

Bei statisch unbestimmten Systemen dagegen ist die Mechanismusbedingung erst erfüllt, wenn mehrere Querschnitte plastifiziert sind.

Die Schnittkraftverteilung nach der Plastizitätstheorie hängt somit von den Gleichgewichtsbedingungen und von der Lage der plastifizierten Querschnitte und deren Querschnittswiderständen ab. Daraus ist ersichtlich, dass die Frage nach der Traglast eines Systems ebenso wie die dazugehörende Schnittkraftverteilung erst mit Kenntnis der Querschnittswiderstände beantwortet werden kann.

Dies soll an einem einfachen Beispiel, einem geraden einseitig eingespannten Träger mit konstantem plastischem Querschnittswiderstand $M_{\omega p}$ ' gezeigt werden. Für die Bestimmung der Traglast und der dazugehörenden Schnittkraftverteilung dient die in den Gleichgewichtsbedingungen vorhandene und in (12) dargestellte formelie Analogie zwischen Wölbtorsion und Balkenbiegung. Der Einfluss der Schubbeanspruchung auf die Fliessbedingungen, d.h. auf das plastische wölbmoment $M_{\omega p}$ wird der Einfachheit halber vernachlässigt. 


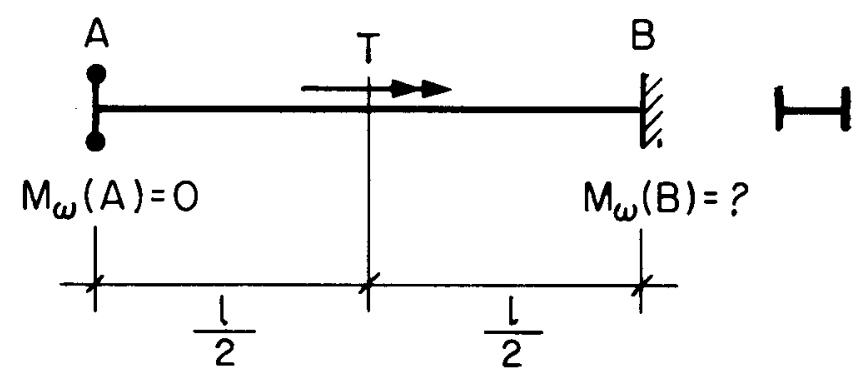

Analogiebalken:

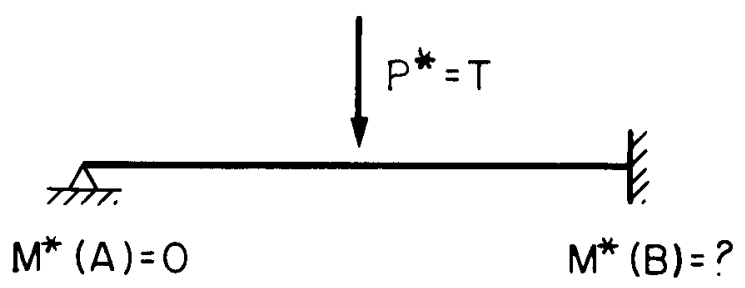

Vermuteter Mechanismus:

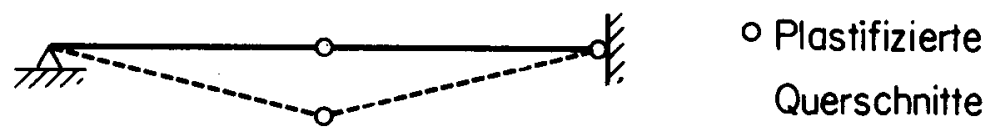

Entsprechende Schnittkraftverteilung:
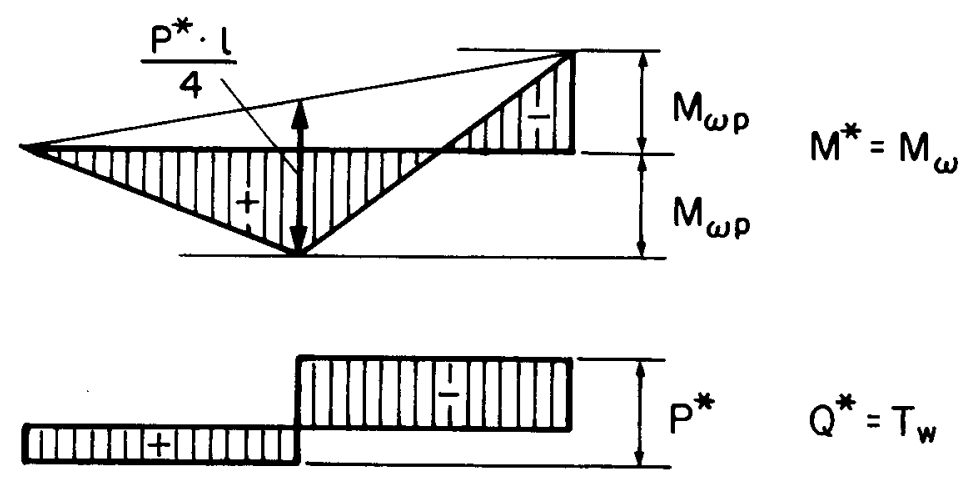
Die zum eingezeichneten $M^{*}$-Diagramm gehörende Last $P^{*}$ ergibt sich mit Hilfe der Gleichgewichtsbedingungen zu:

$$
T=P^{*}=6 \cdot \frac{{ }^{M} \omega p}{1}
$$

Wie man sieht, verletzt die erhaltene Schnittkraftverteilung nirgends die Plastizitätsbedingung. Also sind alle drei Bedingungen für eine plastische Lösung erfüllt, und die obige Last $P^{*}$ ist mit der Traglast $P_{P}^{*}$ identisch.

$$
T_{p}=P_{p}^{*}=6 \cdot \frac{M}{1}
$$

Im untersuchten Beispiel hängt die Traglast vom plastischen Wölbmoment $M_{\omega}$ ab. Im allgemeinen Fall ist sie eine Funktion der plastischen Querschnittswiderstände, deren Bestimmung zur eigentlichen Hauptaufgabe des Problems wird und womit sich die restlichen Kapitel der vorliegenden Arbeit befassen. Darin wird untersucht, welche Belastungen, d.h. welche Schnittkräfte zur Plastifizierung eines dünnwandigen offenen Querschnittes führen und was für Querschnittsmechanismen dabei auftreten. Die Frage nach dem Querschnittswiderstand ist folglich gleichbedeutend mit der Frage nach der Traglast eines durch Schnittkräfte beanspruchten Querschnittes.

Nun ist es im allgemeinen nicht mäglich, alle drei Bedingungen für eine plastische Lösung in einem Rechengang zu befriedigen. Die praktische Berechnung erfolgt aus diesem Grunde nach einem der beiden Grenzwertsätze der Plastizitätstheorie, welche der Literatur, z.B. [5], [6], entnommen werden können.

\section{St_atis scher Grenzzwertsatz:}

Jede Belastung, zu der sich ein stabiler, statisch zulässiger Spannungszustand angeben lässt, stellt eine untere Schranke der Traglast dar.

Ein Spannungszustand wird als statisch zulässig bezeichnet, wenn unter einer Gruppe von äusseren und inneren Kräften jeder Teil des betrachteten Systems im Gleichgewicht ist. Ein solcher Spannungszustand wird als stabil bezeichnet, wenn nirgends die Plastizitätsbedingung verletzt wird.

Ein Gleichgewichtszustand, für den die Plastizitätsbedingung erfüllt ist, liefert also einen unteren Grenzwert der Traglast. 


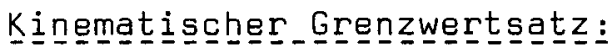

Jede Belastung, zu der sich ein instabiler, kinematisch zulässiger Bewegungszustand bzw. Mechanismus angeben lässt, stellt eine obere Schranke der Traglast dar.

Ein Bewegungszustand wird als kinematisch zulässig bezeichnet, wenn er die kinematischen, d.h. geometrischen Bindungen des systems nicht verletzt. Ein solcher Bewegungszustand wird als instabil bezeichnet, wenn die dabei verrichtete Leistung oder, was bei kleinen Verschiebungen auf dasselbe herauskommt, die dabei verrichtete Arbeit gerade noch verschwindet.

Ein Mechanismus, für den die geleistete Arbeit verschwindet, liefert also einen oberen Grenzwert der Traglast. 
4. PLASTISCHER WIDERSTAND VON QUERSCHNITTEN AUS HOMOGENEN ISOTROPEN MATERIALIEN

\subsection{Bruchmodell und Materialverhalten}

Vorerst sei betont, dass die Gleichgewichtsbedingungen nach der Theorie 1. Ordnung am unverformten System aufgestellt werden. Folglich bleibt die Anwendung auf Querschnitte beschränkt, bei denen ein vorzeitiges Versagen durch Instabilität ausgeschlossen ist. Als Beispiele für solche Querschnitte mögen die auf dem Gebiet des Stahlbaues üblichen Walzprofile dienen.

Für die Berechnung des plastischen Widerstandes wird zwecks einfacher mathematischer Behandlung folgendes diskretisierte Bruchmodell angenommen: Der Querschnitt wird, falls er gekrümmt ist, durch einen polygonalen Querschnitt angenähert, indem man ihn in ebene Elemente unterteilt. Wie aus bild 24 hervorgeht, erstreckt sich ein solches Element i über den Querschnittsbereich $\Delta s_{i}$

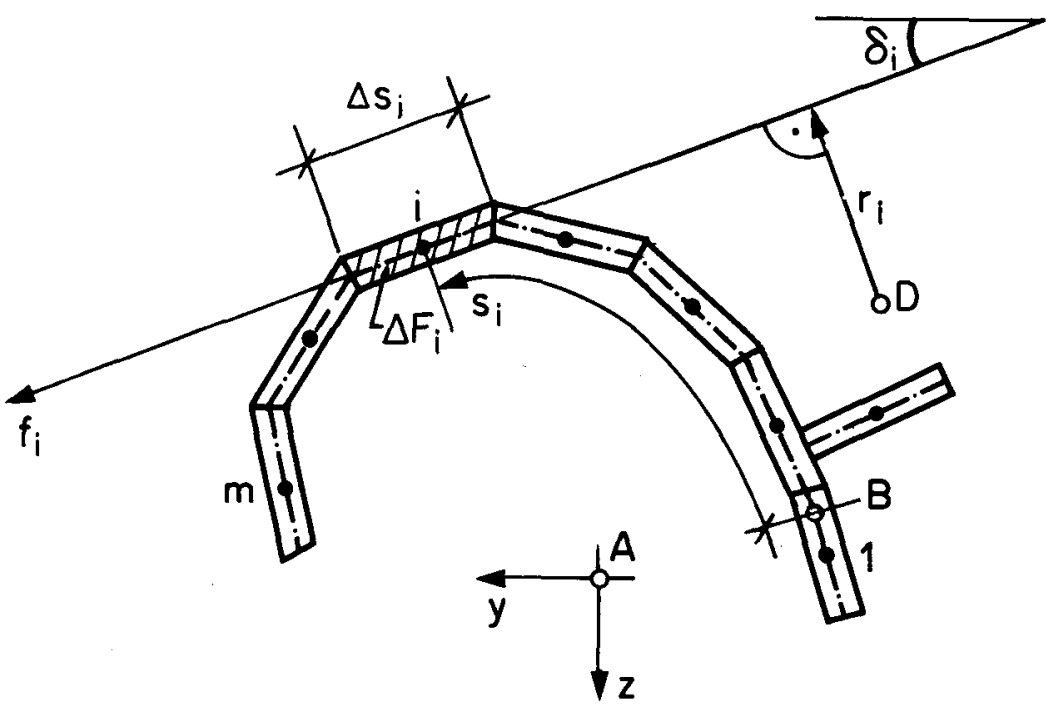


Um trotz dieser Diskretisation gute Resultate zu bekommen, muss eine genügende Anzahl Elemente gewählt werden. Es ist jedoch zu beachten, dass eine zu feine Einteilung lediglich den Rechenaufwand erhöht, ohne das Resultat entscheidend zu verbessern.

Das gewählte Bruchmodell ermöglicht es, mit sogenannten Elementkräften statt mit Spannungen zu arbeiten. Pro Element wirken eine Längskraft $Z_{i}$ und eine Querkraft $Q_{i}$ tangential zur Mittellinie des Querschnittes.

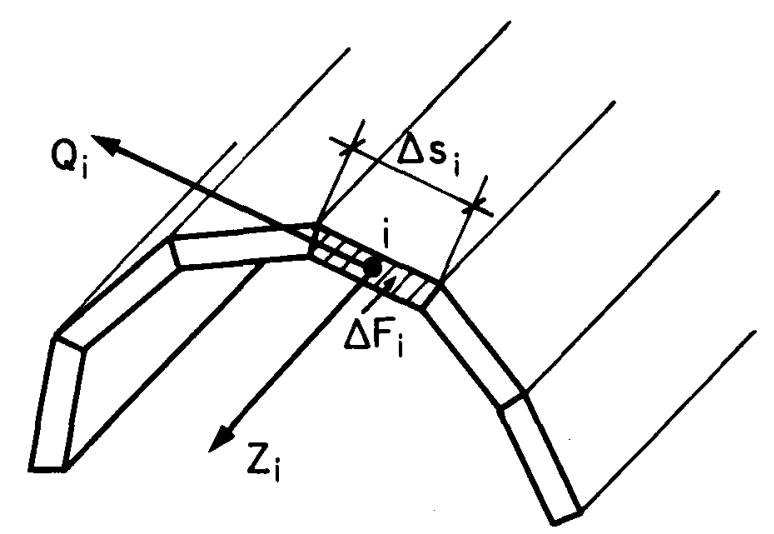

Bild 25

Für die Elementkräfte $Z_{i}$ und $Q_{i}$ gelten folgende Beziehungen:

$$
\begin{aligned}
& z_{i}=\left(\sigma_{x} \cdot \Delta F\right)_{i} \\
& Q_{i}=(s \cdot \Delta s)_{i}=\left(\tau_{x s} \cdot \Delta F\right)_{i}
\end{aligned}
$$

In diesem Kapitel werden nur Querschnitte aus solchen Materialien behandelt, welche homogene und isotrope Eigenschaften aufweisen, wofür man Stahl als wichtigstes Beispiel nennen kann. Die bekannte von Mises'sche Fliessbedingung gibt für derartige Materialien eine brauchbare Näherung des plastischen Verhaltens und wird hier aus diesem Grunde verwendet.

Mit der aus dem statischen Zugversuch entnommenen Fliessspannung $\sigma_{f}$ kann die von Mises'sche Fliessbedingung für das Element i wie folgt formuliert werden:

$$
\left(\sigma_{x}\right)_{i}^{2}+3 \cdot\left(\tau_{x s}\right)_{i}^{2}=\sigma_{f}^{2}
$$

$$
\text { oder } Z_{i}^{2}+3 \cdot Q_{i}^{2}=\left(\sigma_{f} \cdot \Delta F_{i}\right)^{2}
$$


Diese nichtlineare Fliessbedingung stellt in der $Z_{i}{ }^{-Q_{i}}$-Ebene eine Ellipse dar. Für die numerische Behandlung des Traglastproblems mit Hilfe der Iinearen Programmierung ist es notwendig, die Gleichung (35) zu linearisieren. Am einfachsten geschieht dies, indem man die erwähnte Ellipse durch ein eingeschriebenes Polygon approximiert, womit man sich bezüglich der Fliessbedingung (35) auf der sicheren seite befindet.

Wie aus Bild 26 hervorgeht, wird die Ellipse nach von Mises im folgenden durch ein eingeschriebenes Sechseck angenähert.
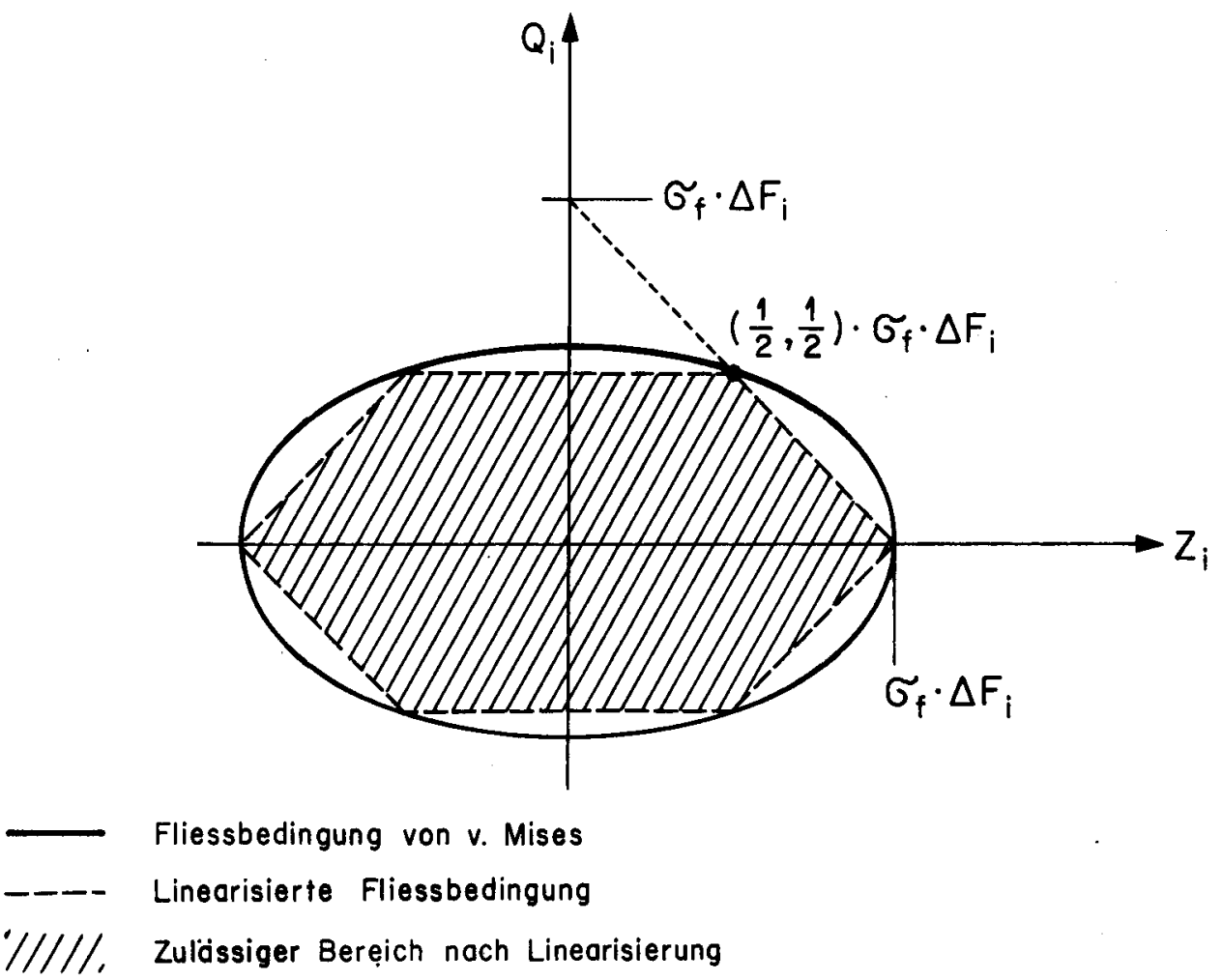

Bild 26

Formelmässig lässt sich die dargestellte polygonale Fliessbedingung für das Element $i$ durch sechs lineare Gleichungen ausdrücken:

$$
\begin{aligned}
& \sigma_{f} \cdot \Delta F_{i}-2 Q_{i}=0 \\
& \sigma_{f} \cdot \Delta F_{i}+2 Q_{i}=0 \\
& \sigma_{f} \cdot \Delta F_{i}-z_{i}-Q_{i}=0 \\
& \sigma_{f} \cdot \Delta F_{i}-z_{i}+Q_{i}=0 \\
& \sigma_{f} \cdot \Delta F_{i}+z_{i}-Q_{i}=0 \\
& \sigma_{f} \cdot \Delta F_{i}+z_{i}+Q_{i}=0
\end{aligned}
$$

Befindet sich der Beanspruchungspunkt $\left(Z_{i}, Q_{i}\right)$ auf der Fliessfigur, so ist das Element $i$ plastifiziert und kann sich verformen. Dabei sind verschie- 
dene Mechanismen denkbar. Bei Verwendung eines sechseckigen Fliesspolygons ist es möglich, alle diese Mechanismen durch nur sechs verschiedene Verformungsparameter darzustellen, da die Verformungsvektoren nach der Theorie des plastischen Potentials immer senkrecht auf derr Fliessfigur stehen müssen.

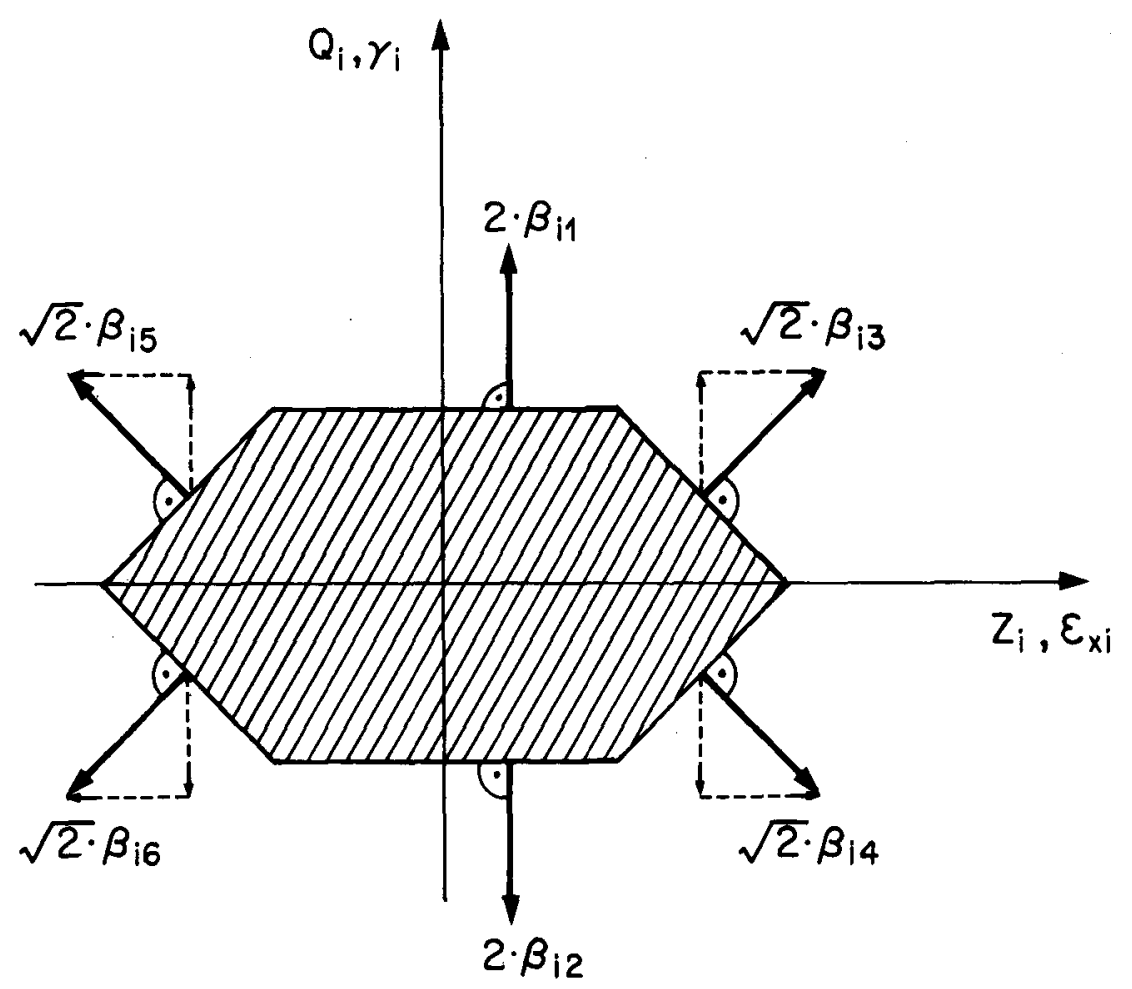

Bild 27

Absichtlich werden hier die Verformungsparameter mit den Konstanten $\sqrt{2}$ bzw. 2 eingeführt. Diese Konstanten sind unwesentlich, sie werden nur darum eingeführt, um später numerisch einfachere Ausdrücke zu erhalten.

Man kann die gesuchten kinematischen Grössen, die Längsdehnungen $\varepsilon_{x i}$ und die Schiebungen $\gamma_{i}$ der Elemente, als Summe der entsprechenden Projektionen der Verformungsparameter angeben, wie sie in Bild 27 eingezeichnet sind.

$$
\begin{aligned}
& \varepsilon_{x i}=\beta_{i 3}+\beta_{i 4}-\beta_{i 5}-\beta_{i 6} \\
& \gamma_{i}=2 \beta_{i 1}-2 \beta_{i 2}+\beta_{i 3}-\beta_{i 4}+\beta_{i 5}-\beta_{i 6}
\end{aligned}
$$

Die Verformungsparameter sind nichtnegative Variablen, und jeder dieser Parameter ist, falls er nicht verschwinden soll, einer Gleichung des Systems (36) zugeordnet. Es gelten die Beziehungen: 


$$
\begin{aligned}
& \beta_{i 1}>0 \rightarrow \quad 2 Q_{i}=\sigma_{f} \cdot \Delta F_{i} \\
& \beta_{i 2}>0 \rightarrow-2 Q_{i}=\sigma_{f} \cdot \Delta F_{i} \\
& \beta_{i 3}>0 \rightarrow Z_{i}+Q_{i}=\sigma_{f} \cdot \Delta F_{i} \\
& \beta_{i 4}>0 \rightarrow Z_{i}-Q_{i}=\sigma_{f} \cdot \Delta F_{i} \\
& \beta_{i 5}>0 \rightarrow-Z_{i}+Q_{i}=\sigma_{f} \cdot \Delta F_{i} \\
& \beta_{i 6}>0 \rightarrow-Z_{i}-Q_{i}=\sigma_{f} \cdot \Delta F_{i}
\end{aligned}
$$

Bei starrem Element $i$ befindet sich der Beanspruchungspunkt $\left(Z_{i}, Q_{i}\right)$ innerhalb der Fliessfigur. Somit wird keine Gleichung des Systems (36) befriedigt, und die Verformungsparameter sind alle gleich Null. Fliesst das Element $i$, so müssen eine oder zwei Gleichungen des Systems (36) erfüllt sein, da der Beanspruchungspunkt $\left(Z_{i}, Q_{i}\right)$ auf der Fliessfigur liegt. In diesem Fall nehmen nach (38) ein bzw. zwei Verformungsparameter einen von Null verschiedenen positiven wert an.

Die Berechnung des Querschnittswiderstandes kann damit in Angriff genommen werden. In der Folge wird mit dem statischen Grenzwertsatz eine untere und mit dem kinematischen Grenzwertsatz eine obere Schranke der für die Plastifizierung des Querschnittes massgebenden Beanspruchung hergeleitet. (Obere Schranke bezüglich der linearisierten Fliessbedingung (36)!).

\subsection{Statische Methode}

Der statische Grenzwertsatz lässt sich für die Bestimmung einer unteren Schranke des Querschnittswiderstandes wie folgt anwenden:

Unter Einhaltung der Gleichgewichts- und Plastizitätsbedingungen wird jene Beanspruchung $\lambda \cdot\left(N, M_{y}, M_{z}, M_{\omega}, Q_{y}, Q_{z}, T_{w}\right)$ gesucht, für die der Widerstandsfaktor $\lambda$ maximal wird. Dabei wird eine proportionale Steigerung der Beanspruchung angenommen.

\section{Gleichgewichtsbedingungen:}

Mit Einführung des Widerstandsfaktors $\lambda$ sowie der Elementkräfte $Z_{i}$ und $Q_{i}$, für welche die Beziehungen (34) gelten, transformieren sich die Gleichgewichtsbedingungen (15) zu: 


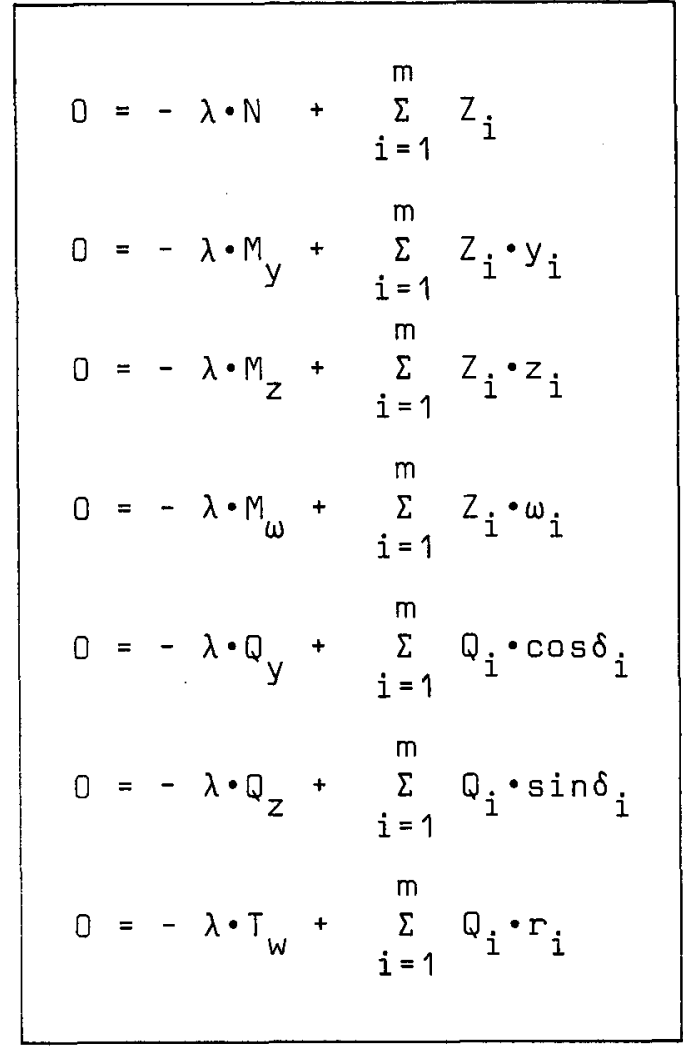

\section{Plastizitätsbedingungen:}

Die Plastizitätsbedingungen verlangen, dass die Beanspruchung jedes einzelnen Elementes kleiner oder gleich ist wie dessen plastischer Widerstand. Der Beanspruchungspunkt $\left(Z_{i}, Q_{i}\right)$ des Elementes $i$ muss dabei im schraffierten, zulässigen Bereich der Fliessfigur von bild 26 bzw. 27 liegen. Mathematisch kann man den zulässigen Bereich durch Ungleichungen beschreiben, die genau wie die Gleichungen (36), welche die Grenze des erwähnten Bereiches darstellen, aufgebaut sind. Diese Ungleichungen lauten mit Einführung nichtnegativer Schlupfvariablen $s_{i 1}$ bis $s_{i 6}$ folgendermassen:

$$
\begin{aligned}
& s_{i 1}=\sigma_{f} \cdot \Delta F_{i}-2 Q_{i} \geq 0 \\
& s_{i 2}=\sigma_{f} \cdot \Delta F_{i}+2 Q_{i} \geq 0 \\
& s_{i 3}=\sigma_{f} \cdot \Delta \Delta_{i}-z_{i}-Q_{i} \geq 0 \\
& s_{i 4}=\sigma_{f} \cdot \Delta F_{i}-z_{i}+Q_{i} \geq 0 \\
& s_{i 5}=\sigma_{f} \cdot \Delta F_{i}+z_{i}-Q_{i} \geq 0 \\
& s_{i 6}=\sigma_{f} \cdot \Delta F_{i}+z_{i}+Q_{i} \geq 0
\end{aligned}
$$




\section{Optimierung:}

Die Gleichgewichtsbedingungen (39), die Plastizitätsbedingungen (40) sowie die Zielfunktion $\lambda \rightarrow$ Maximum werden im Tableau de Bildes 28 zusammengestellt.

In der Basis dieses Tableaus stehen die Nullen aus den Gleichgewichtsbedingungen und die Schlupfvariablen aus den Plastizitätsbedingungen, am Kopf die freien unabhängigen Variablen $Z_{i}$ und $Q_{i}$.

Die Zielfunktion findet man in der ersten Zeile des Tableaus.

Die statische Methode der Plastizitätstheorie führt also für das betrachtete Bruchmodell zu einem Maximumproblem der linearen Optimierung, zu dessen Lösung der Simplex-Algorithmus verwendet werden kann. 


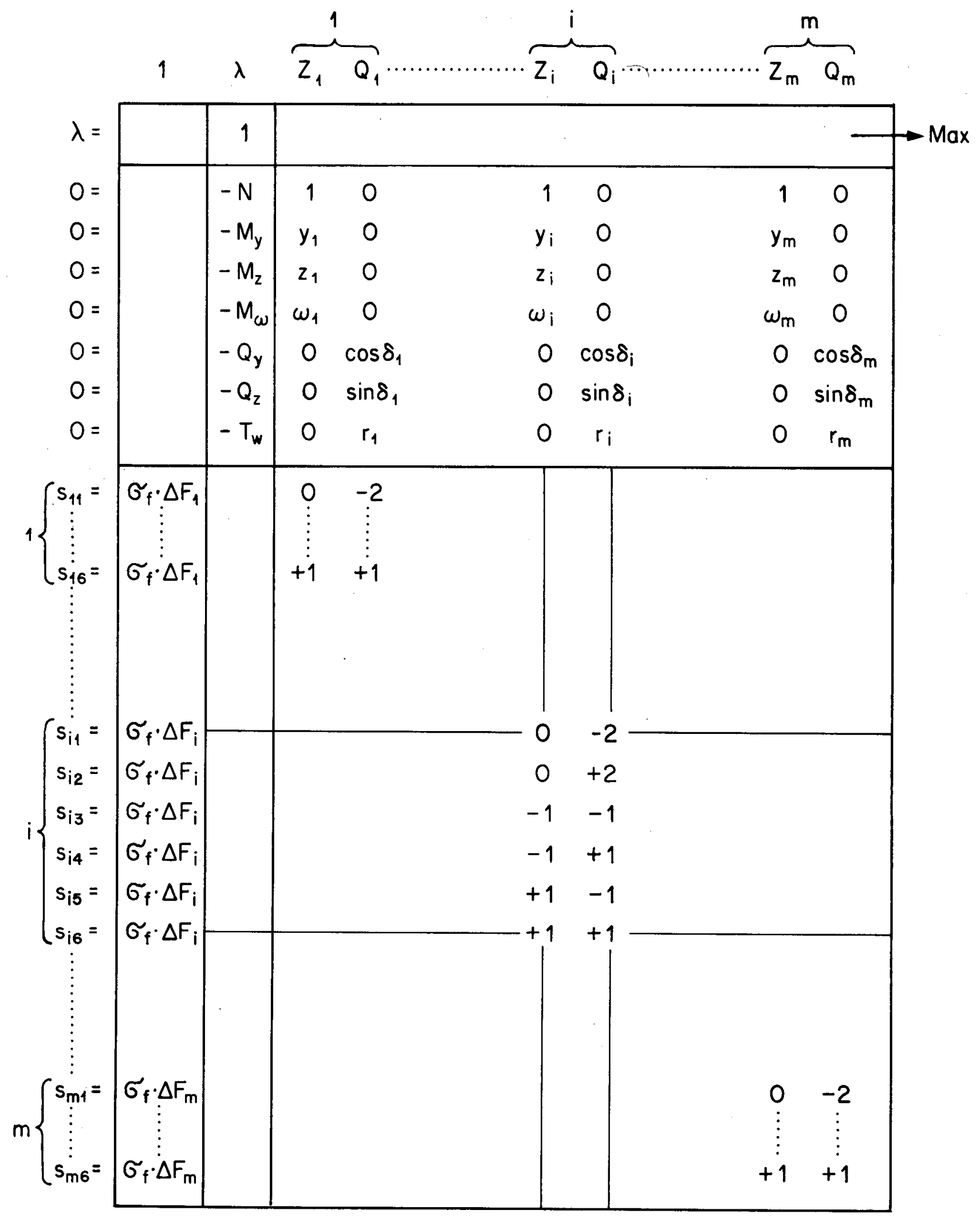


Für die Bestimmung einer oberen Schranke des Querschnittswiderstandes kann der kinematische Grenzwertsatz wie folgt angewandt werden:

Unter Annahme proportionaler Steigerung der Beanspruchung $\lambda \cdot\left(N, M_{y}, M_{z}, M_{w}, Q_{y}, Q_{z}, T_{w}\right)$ wird jener Querschnitts-Mechanismus gesucht, für den die geleistete Arbeit verschwindet und der Widerstandsfaktor $\lambda$ minimal wird.

\section{Mechanismusbedingungen:}

Die kinematischen Bedingungen für das Element $i$ können den im Abschnitt 2.3 hergeleiteten Gleichungen (19) und (22) entnommen werden. Die Ableitungen nach der Koordinate $x$ werden durch Striche angedeutet.

$$
\begin{aligned}
& \varepsilon_{x i}=u^{\prime}-v^{\prime \prime} \cdot y_{i}-w^{\prime \prime} \cdot z_{i}-\vartheta " \cdot w_{i} \\
& \gamma_{i}=\gamma_{y} \cdot \cos \delta_{i}+\gamma_{z} \cdot \sin \delta_{i}+\gamma_{\vartheta} \cdot r_{i}
\end{aligned}
$$

Aus diesen Gleichungen erhält man bei Verwendung der Beziehungen (37), durch die das Fliessgesetz formuliert ist, die gesuchten Mechanismusbedingungen.

$$
\begin{aligned}
& u^{\prime}-v^{\prime \prime} \cdot y_{i}-w^{n} \cdot z_{i}-\vartheta " \cdot \omega_{i}-\beta_{i 3}-\beta_{i 4}+\beta_{i 5}+\beta_{i 6}=0 \\
& \gamma_{y} \cdot \cos \delta_{i}+\gamma_{z} \cdot \sin \delta_{i}+\gamma_{\vartheta} \cdot r_{i}-2 \beta_{i 1}+2 \beta_{i 2}-\beta_{i 3}+\beta_{i 4}-\beta_{i 5}+\beta_{i 6}=0
\end{aligned}
$$

\section{Arbeitsbedingung:}

Die Arbeitsbedingung besagt, dass beim Verschiebungszustand eines Mechanismus keine Arbeit geleistet wird.

$$
A_{a}+A_{i}=0
$$

$$
\begin{aligned}
& A_{a}: \text { Arbeit der äusseren Kräfte (hier: Schnittkräfte) } \\
& A_{i}: \text { Arbeit der inneren Kräfte (Spannungen) }
\end{aligned}
$$

Für den aus den Dehnungen (10 und den Schiebungen (22) gebildeten Verschiebungszustand 


$$
\begin{aligned}
& \varepsilon_{x}=u^{\prime}-v^{\prime \prime} \cdot y-w^{\prime \prime} \cdot z-\vartheta " \cdot \omega \\
& \gamma=\gamma_{y} \cdot \cos \delta+\gamma_{z} \cdot \sin \delta+\gamma_{\vartheta} \cdot r
\end{aligned}
$$

erhält man folgenden Ausdruck für die Arbeit der inneren Kräfte pro Einheitslänge $\Delta x=1$ :

$$
\begin{aligned}
-A_{i}= & \int_{a}^{e} \sigma_{x} \cdot \varepsilon_{x} \cdot d \cdot d s \quad+\int_{a}^{e} \tau \cdot \gamma \cdot d \cdot d s= \\
= & \int_{a}^{e} \sigma_{x} \cdot \varepsilon_{x} \cdot d \cdot d s \quad+\int_{a}^{e} s \cdot \gamma \cdot d s \\
-A_{i}= & \int_{a}^{e} \sigma_{x} \cdot\left[u^{\prime}-v^{n} \cdot y-w^{n} \cdot z-\vartheta n \cdot w\right] \cdot d \cdot d s+ \\
& +\int_{a}^{e} s \cdot\left[\gamma_{y} \cdot \cos \delta \quad+\gamma_{z} \cdot \sin \delta+\gamma_{\vartheta} \cdot r\right] \cdot d s \\
-A_{i}= & u^{\prime} \cdot \int_{a}^{e} \sigma_{x} \cdot d \cdot d s+v^{n} \cdot \int_{a}^{e} \sigma_{x} \cdot y \cdot d \cdot d s- \\
& -w^{n} \cdot \int_{a}^{e} \sigma_{x} \cdot z \cdot d \cdot d s-\vartheta^{n} \cdot \int_{a}^{e} \sigma_{x} \cdot \omega \cdot d \cdot d s+ \\
& +\gamma_{y} \cdot \int_{a}^{e} s \cdot c o s \delta \cdot d s+\gamma_{z} \cdot \int_{a}^{e} s \cdot \sin \delta \cdot d s+ \\
& +\gamma_{\vartheta} \cdot \int_{a}^{e} s \cdot r \cdot d s
\end{aligned}
$$

Die entsprechende Arbeit der am Querschnitt angreifenden äusseren Kräfte, d.h. der Beanspruchung ( $\left.N, M_{y}, M_{z}, M_{\omega}, Q_{y}, Q_{z}, T_{w}\right)$, kann daraus mit Hilfe der Beziehungen (15) abgeleitet werden.

$$
\begin{aligned}
A_{a}=-A_{i}= & u^{\prime} \cdot N-v " \cdot M_{y}-w " \cdot M_{z}-\vartheta " \cdot M_{\omega}+ \\
& +\gamma_{y} \cdot Q_{y}+\gamma_{z} \cdot Q_{z}+\gamma_{\vartheta} \cdot T_{w}
\end{aligned}
$$

Diese Arbeit der äusseren Kräfte vergrössert sich bei proportionaler Steigerung der Beanspruchung um den Widerstandsfaktor $\lambda$.

$$
\begin{aligned}
A_{a}= & \lambda \cdot\left[u^{\prime} \cdot N-v^{\prime \prime} \cdot M_{y}-w^{n} \cdot M_{z}-\vartheta " \cdot M_{\omega}+\right. \\
& \left.+\gamma_{y} \cdot Q_{y}+\gamma_{z} \cdot Q_{z}+\gamma_{\vartheta} \cdot T_{w}\right]
\end{aligned}
$$


Bei der vorausgesetzten Annahme eines starr-idealplastischen Materials ist die Dissipationsarbeit gleich der negativen Arbeit der inneren Kräfte.

$$
A_{d}=-A_{i}=\int_{a}^{e} \sigma_{x} \cdot \varepsilon_{x} \cdot d \cdot d s+\int_{a}^{e} s \cdot \gamma \cdot d s
$$

Für das angenommene Bruchmodell, wie es zu Beginn dieses Kapitels beschrieben ist, kann das Integral der Dissipationsarbeit durch eine Summe der Dissipationsarbeiten der einzelnen Elemente ersetzt werden.

$$
A_{d}=\sum_{i=1}^{m}\left[Z_{i} \cdot \varepsilon_{x i}+Q_{i} \cdot \gamma_{i}\right]
$$

Wird der Verschiebungszustand $\varepsilon_{x i}, \gamma_{i}$ des Elementes $i$ durch das Fliessgesetz (37)

$$
\begin{aligned}
& \varepsilon_{x i}=\beta_{i 3}+\beta_{i 4}-\beta_{i 5}-\beta_{i 6} \\
& \gamma_{i}=2 \beta_{i 1}-2 \beta_{i 2}+\beta_{i 3}-\beta_{i 4}+\beta_{i 5}-\beta_{i 6}
\end{aligned}
$$

ausgedrückt, so ergibt sich:

$$
\begin{aligned}
A_{d}= & \sum_{i=1}^{m}\left[Z_{i} \cdot\left(\beta_{i 3}+\beta_{i 4}-\beta_{i 5}-\beta_{i 6}\right)+\right. \\
& \left.+Q_{i} \cdot\left(2 \beta_{i 1}-2 \beta_{i 2}+\beta_{i 3}-\beta_{i 4}+\beta_{i 5}-\beta_{i 6}\right)\right] \\
A_{d}= & \sum_{i=1}^{m}\left(2 Q_{i} \cdot \beta_{i 1}+\left(-2 Q_{i}\right) \cdot \beta_{i 2}+\right. \\
& +\left(Z_{i}+Q_{i}\right) \cdot \beta_{i 3}+\left(Z_{i}-Q_{i}\right) \cdot \beta_{i 4}+ \\
& \left.+\left(-Z_{i}+Q_{i}\right) \cdot \beta_{i 5}+\left(-Z_{i}-Q_{i}\right) \cdot \beta_{i 6}\right]
\end{aligned}
$$

Diese Summe lässt sich mit Hilfe der Beziehungen (3B) folgendermassen vereinfachen:

$$
A_{d}=\sigma_{f} \cdot \sum_{i=1}^{m} \Delta F_{i} \cdot\left(\beta_{i 1}+\beta_{i 2}+\beta_{i 3}+\beta_{i 4}+\beta_{i 5}+\beta_{i 6}\right)
$$

Setzt man die erhaltenen Ausdrücke (45) und (46) in die Arbeitsbedingung $A_{a}=-A_{i}$ bzw. $A_{a}=A_{d}$ ein. 


$$
\begin{aligned}
\lambda \cdot\left[u^{\prime} \cdot N\right. & \left.-\ldots \ldots+\gamma_{\vartheta} \cdot T_{w}\right]= \\
& =\sigma_{f} \cdot \sum_{i=1}^{m} \Delta F_{i} \cdot\left(\beta_{i 1}+\ldots \ldots+\beta_{i b}\right)
\end{aligned}
$$

so erhält man daraus den zu minimierenden widerstandsfaktor:

$$
\lambda=\frac{\sigma_{f} \sum_{i=1}^{m} \Delta F_{i} \cdot\left(\beta_{i 1}+\ldots \ldots+\beta_{i 6}\right)}{u^{\prime} \cdot N-\ldots \ldots+\gamma_{\vartheta} \cdot T_{w}}
$$

Nach der Plastizitätstheorie kann der absolute Betrag der Verformungen einen beliebigen Wert > 0 annehmen. Somit ist es gestattet, den in (47) vorkommenden Nenner gleich Eins zu setzen.

Schliesslich bekommt man folgende Gleichung zur Bestimmung des Widerstandsfaktors $\lambda$

$$
\lambda=\sigma_{f} \cdot \sum_{i=1}^{m} \Delta F_{i} \cdot\left(\beta_{i 1}+\beta_{i 2}+\beta_{i 3}+\beta_{i 4}+\beta_{i 5}+\beta_{i 6}\right)
$$

mit der Nebenbedingung:

$$
u \cdot \cdot N-v " \cdot M_{y}-w " \cdot M_{z}-\vartheta " \cdot M_{w}+\gamma_{y} \cdot Q_{y}+\gamma_{z} \cdot Q_{z}+\gamma_{\vartheta} \cdot T_{w}=1
$$

\section{Optimierung:}

Die Mechanismusbedingungen (42) und die aus der Arbeitsbedingung $A_{a}+A_{i}=0$ bzw. $A_{a}=A_{d}$ erhaltenen Gleichungen (48) und (49) können wiederum in Tableauform dargestellt werden.

Die Zielfunktion bzw. der Widerstandsfaktor $\lambda$ und die Restriktionen sind alles Iineare Funktionen der freien, unabhängigen Variablen $u^{\prime}$ bis $\gamma_{\vartheta}$ und der beschränkten Verformungsparameter $\beta_{i 1}$ bis $\beta_{i 6}$. Die kinematische Methode der Plastizitätstheorie führt also für das betrachtete Bruchmodell zu einem Minimumproblem der linearen Optimierung. 


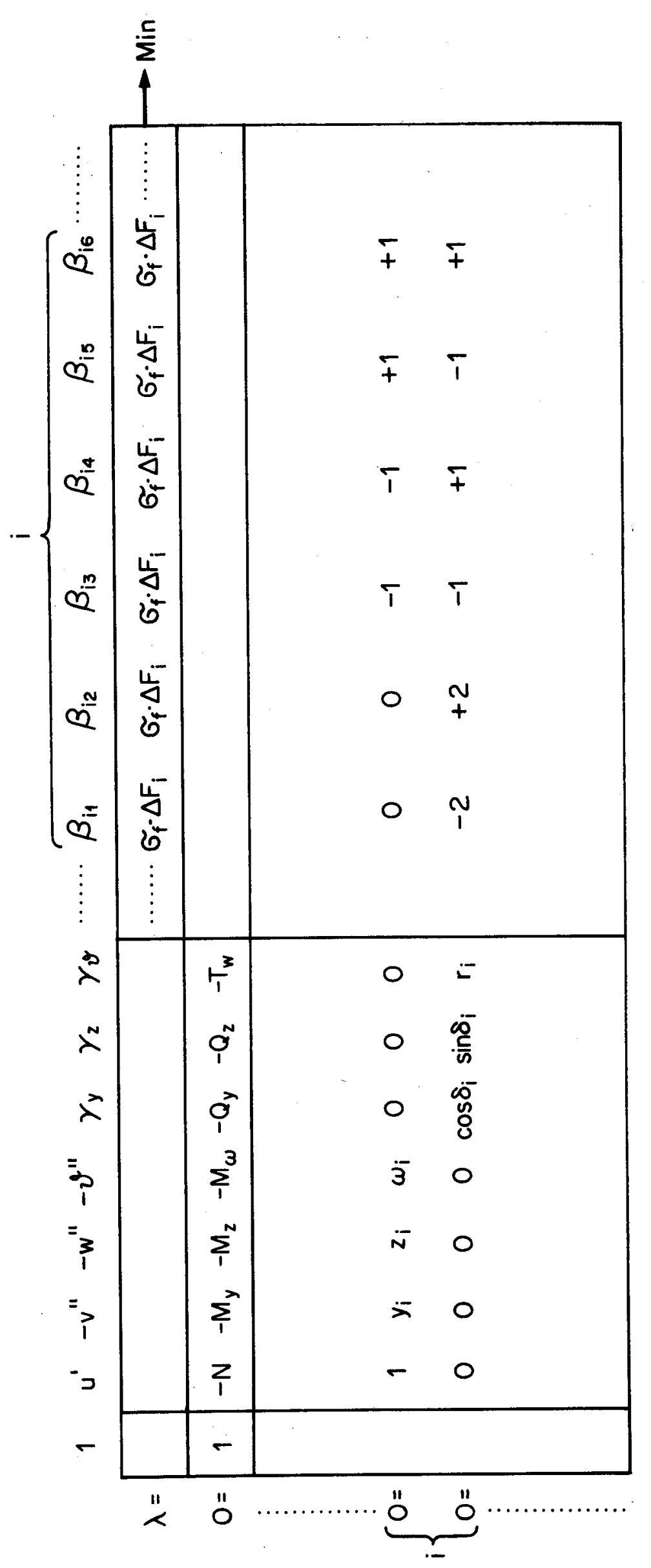


Vergleicht man das Programm der kinematischen Methode (Bild 29) mit demjenigen der statischen Methode (Bild 28), so stellt man fest, dass diese beiden Programme zueinander dual sind. Mit anderen Worten: es ergeben beide Systeme denselben Widerstandsfaktor $\lambda$. Es spielt also keine Rolle, ob die Bestimmung des Querschnittswiderstandes nach der statischen oder nach der kinematischen Methode erfolgt. In jedem Fall erhält man für das beschriebene Bruchmodell ein lineares Programm, zu dessen Lösung die bekannten Methoden der linearen Programmierung, wie sie z.B. in [10] oder [11] zu finden sind, verwendet werden können.

Es sei hier noch einmal daran erinnert, dass das angenommene Bruchmodell nur solange gilt, als die Verformungen keinen wesentlichen Einfluss auf das Gleichgewicht ausüben. Eine Anwendung auf versteifte Blechträger, deren Stegbleche ausbeulen, kommt beispielsweise nicht in Frage. Im allgemeinen ist bei derartigen Trägern nach dem Ueberschreiten der Beullast (im überkritischen Bereich) ein Tragverhalten zu beobachten, welches vorwiegend auf einer Fachwerkwirkung beruht. Die Berechnung der Traglast muss aus diesem Grunde mit einem anderen Bruchmodell als dem beschriebenen erfolgen. Für auf Biegung und Querkraft beanspruchte Stahlträger sind einige derartige Modelle (z.B. [7], [8]) bekannt. Eine Anwendung dieser Modelle auf versteifte Blechträger mit offenen Querschnitten ist naheliegend. Es sei hier nur erwähnt, dass die für den überkritischen Bereich von versteiften Blechträgern gültigen Berechnungsmodelle in ihrem statischen Verhalten grosse Aehnlichkeiten mit dem im folgenden dargestellten Fachwerkmodell für Stahlbetonund Spannbetonbalken aufweisen. 
4.4 Numerisches Beispiel

Gegeben: Querschnitt, Fliessspannung $\sigma_{f}$

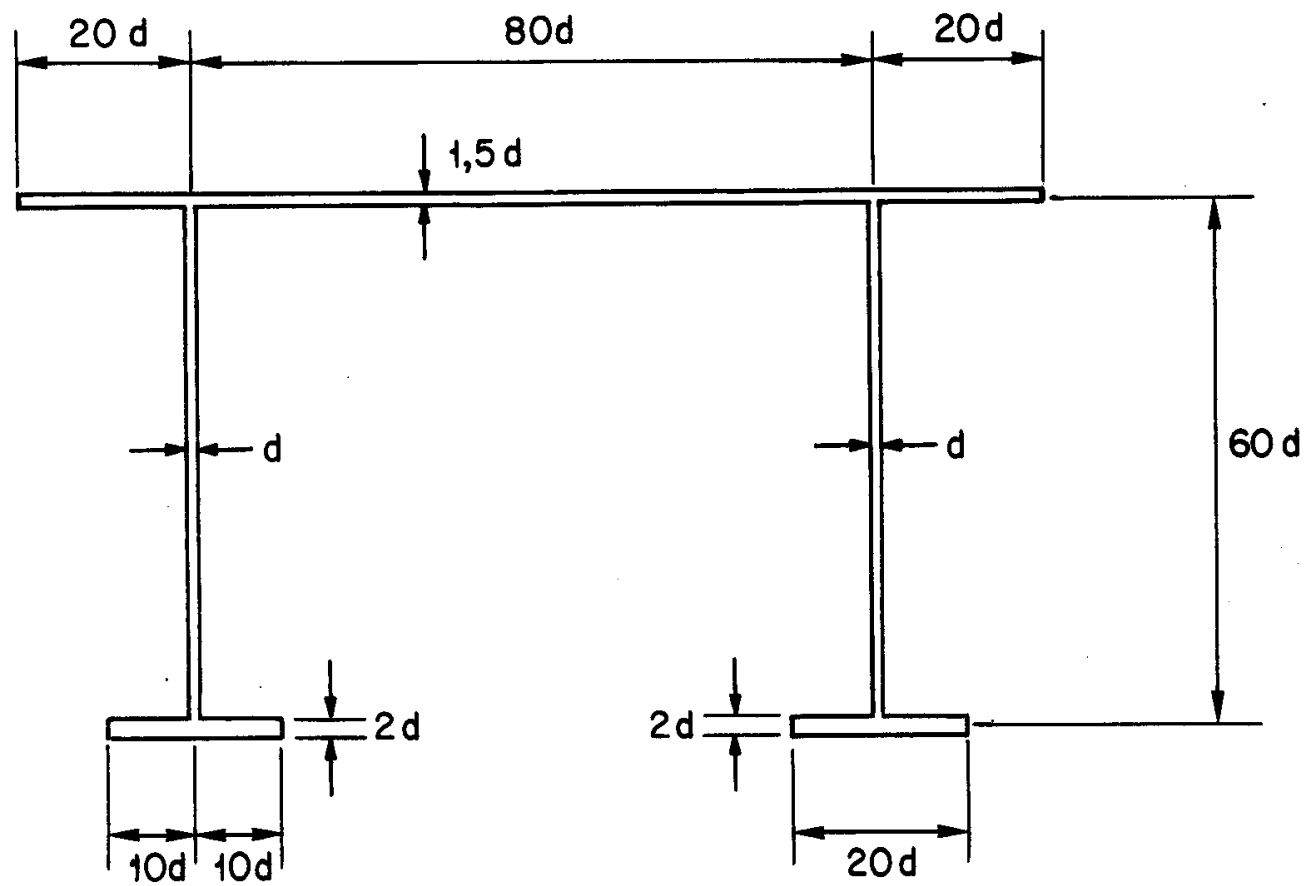

Bild 30

Gesucht: Plastisches Wölbmoment M ${ }_{\omega p}$

Elementeinteilung:

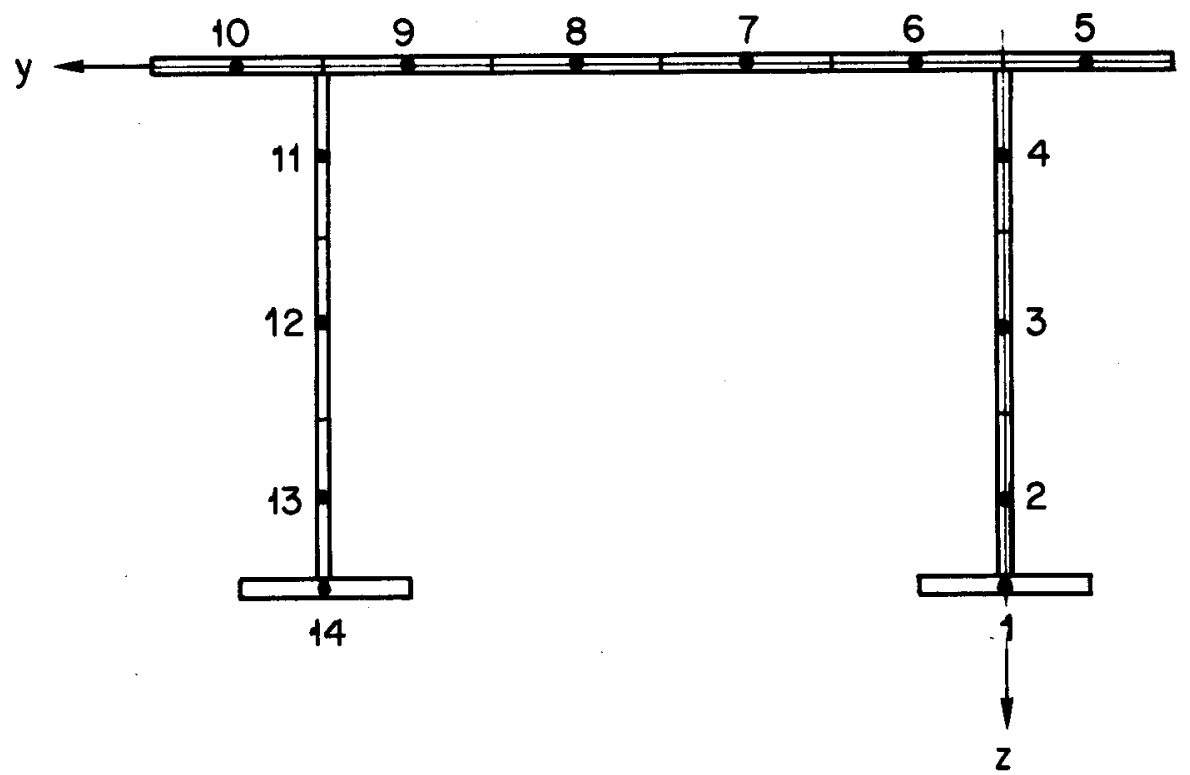




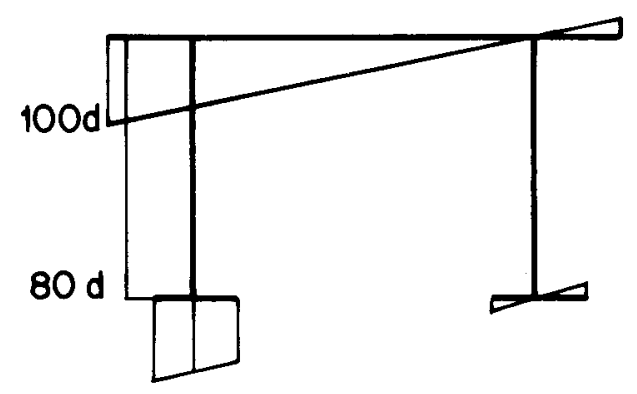

(y)

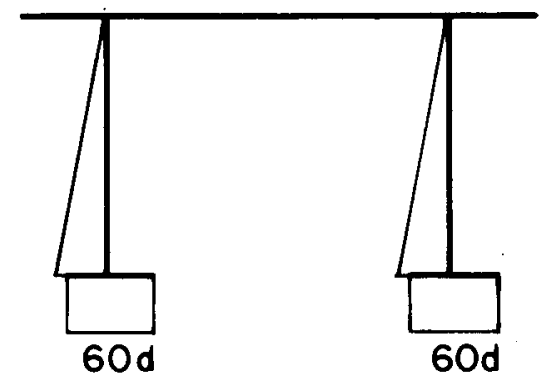

(2)

(4)

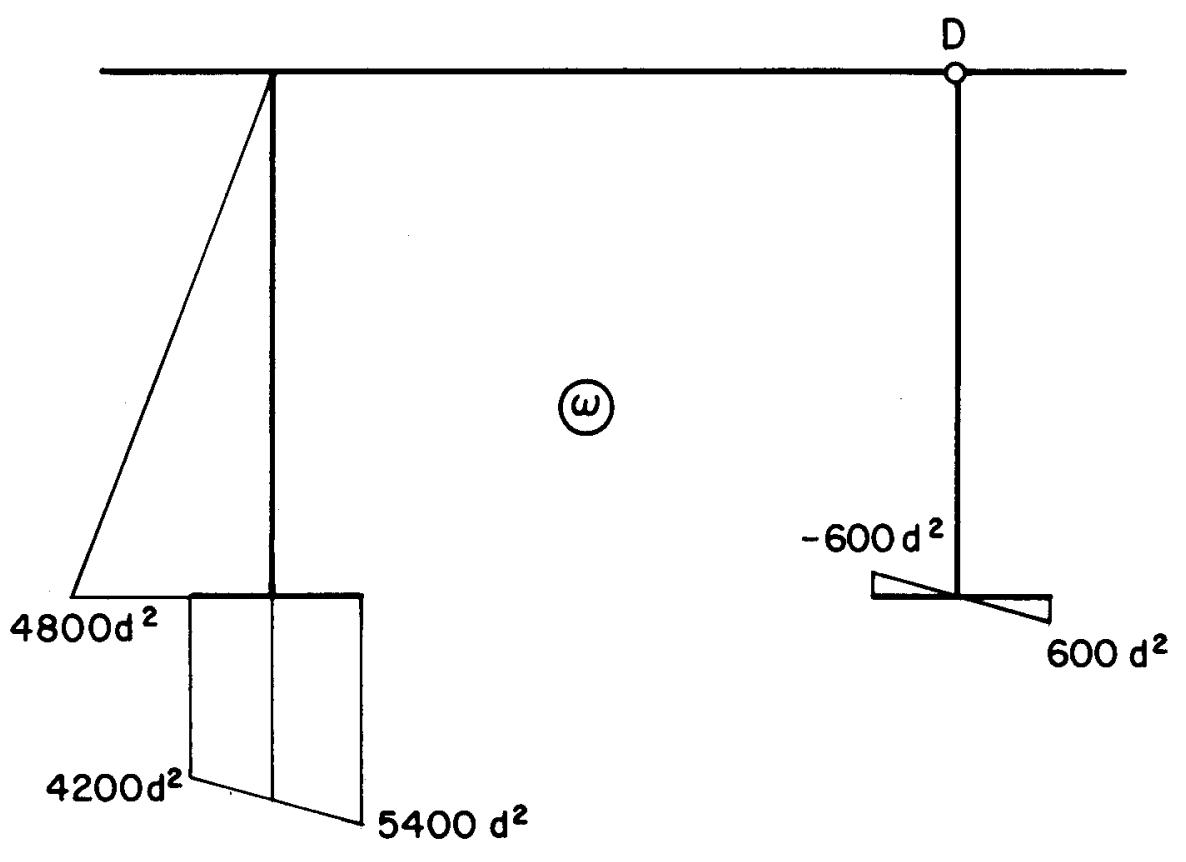

Bild 32 
Näherungslösung nach Abschnitt 4.2 :

$$
\begin{aligned}
& M_{w p}=\lambda \cdot M_{w}=31 \cdot(10 d)^{4} \cdot \sigma_{f} \\
& === \\
& z_{1}=-z_{14}=-40 \cdot d^{2} \cdot \sigma_{f} \\
& z_{2}=-z_{13}=-20 \cdot d^{2} \cdot \sigma_{f} \\
& z_{3}=-z_{12}=-20 \cdot d^{2} \cdot \sigma_{f} \\
& z_{4}=-z_{11}=12,5 \cdot d^{2} \cdot \sigma_{f} \\
& z_{5}=-z_{10}=30 \cdot d^{2} \cdot \sigma_{f} \\
& z_{6}=-z_{9}=30 \cdot d^{2} \cdot \sigma_{f} \\
& z_{7}=-z_{8}=30 \cdot d^{2} \cdot \sigma_{f}
\end{aligned}
$$

Exakter Wert: $M_{\omega p}=31,5 \cdot(10 d)^{4} \cdot \sigma_{f}$

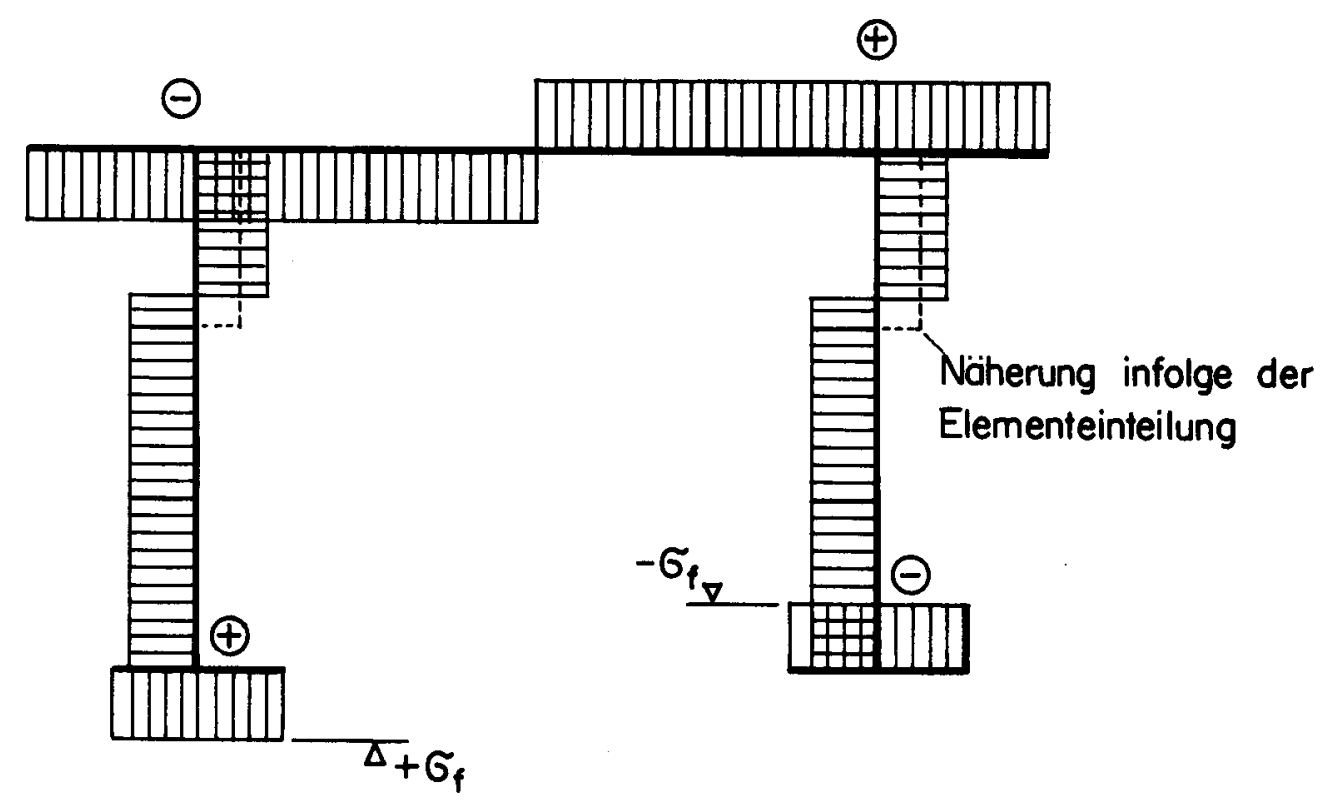


Wölbmoment bei Fliessbeginn:

$M_{\omega f}=16,6 \cdot(10 d)^{4} \cdot \sigma_{f}$

Spannungsverteilung bei Fliessbeginn:

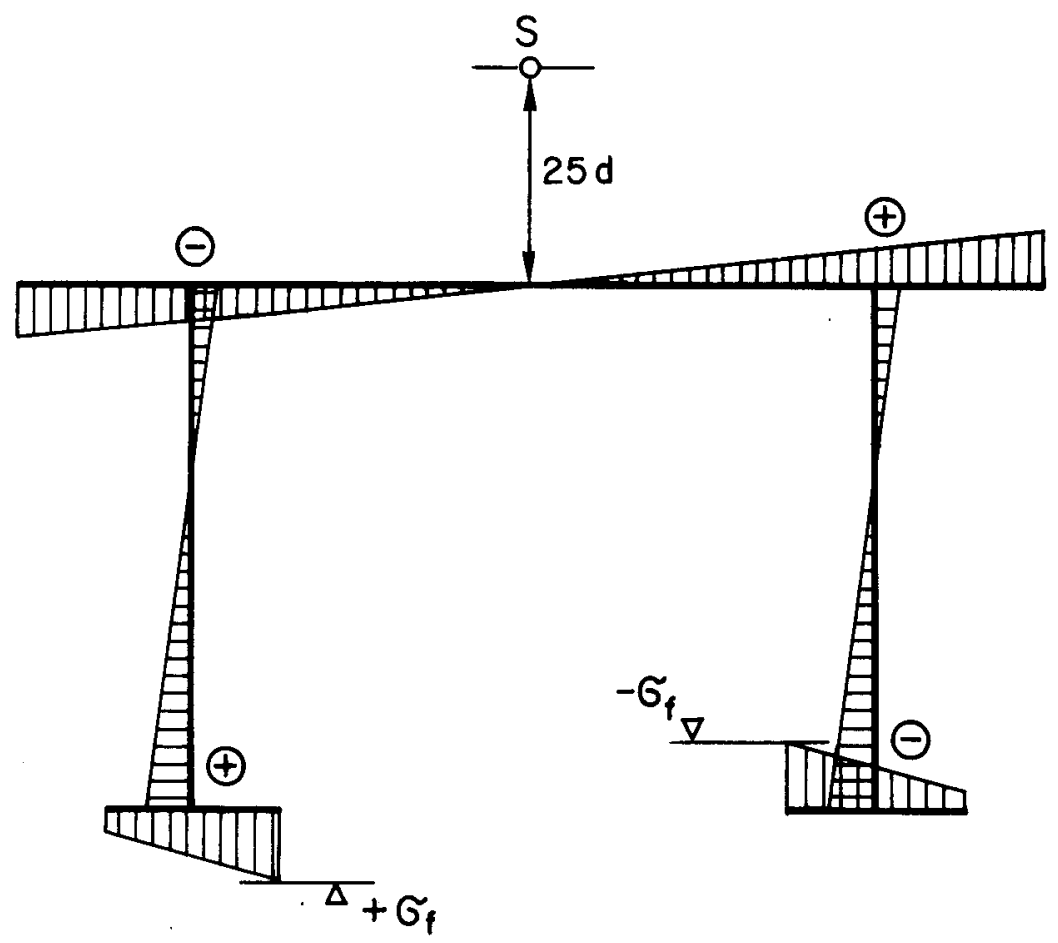

$S=$ Schubmittelpunkt nach Elastizitätstheorie 


\subsection{Bruchmodell und Materialverhalten}

Stahlbeton- und Spannbetonquerschnitte können im wesentlichen in unterarmierte und überarmierte Querschnitte unterteilt werden. Bei unterarmierten Querschnitten wird der Bruch durch Stahlfliessen, bei überarmierten Querschnitten durch Betonbruch verursacht.

Im folgenden werden nur unterarmierte Querschnitte behandelt, bei denen der Beton für das Versagen nicht massgebend ist. Dadurch kann bei Verwendung handelsüblicher Armierungsstähle ein duktiles Bruchverhalten vorausgesetzt werden, was die Anwendung der Plastizitätstheorie zur Bestimmung des Bruchwiderstandes ermöglicht. Die Beschränkung auf unterarmierte Querschnitte ist für die praktische Anwendung nicht sehr einschneidend, da man normalerweise bestrebt ist, überarmierte Tragwerke wegen ihrer Unwirtschaftlichkeit und wegen ihres ausgesprochen spröden Bruchverhaltens zu vermeiden.

Als Bruchmodell wird ein räumliches Fachwerk angenommen, ähnlich wie es Lampert in seiner Dissertation "Bruchwiderstand von Stahlbetonbalken (mit vollen und geschlossenen Querschnitten) unter Torsion und Biegung" [9] verwendet hat. Das Bruchmodell ist ein reines Scheibenmodell, d.h. die Plattenwirkung wird für die theoretische Betrachtung vernachlässigt.

Zur einfachen rechnerischen Erfassung des Bruchwiderstandes wird der Querschnitt idealisiert: Es werden diskret angeordnete Gurtstäbe (Stringer) angenommen, welche die im Querschnitt wirkenden Längskräfte aufzunehmen haben. Dabei sollten, wie im Abschnitt 5.7 gezeigt wird, für den Hauptquerschnitt (vgl. Abschnitt 2.4) vier bis sechs Gurtstäbe gewählt werden, und zwar so, dass trotz der Diskretisation das statische Verhalten des Querschnittes annähernd erfasst wird. Mit anderen Worten, die einzelnen Längseisen werden zu eigentlichen Gurtstäben zusammengefasst. Steht ein solcher Gurtstab unter Druck, so bildet sich um ihn herum eine Betondruckzone, die bei unterarmierten Querschnitten in der Lage ist, die anfallende Druckkraft zu übernehmen. Im allgemeinen ändert sich die Lage der Betondruckresultierenden mit der Grösse der Betondruckzone. Im folgenden wird die Druckkraft vereinfachend an den betreffenden Gurtstab gebunden, d.h. ihre Lage wird in der Rechnung nicht davon abhängig gemacht, ob und wie stark ein Gurtstab auf Druck beansprucht wird. Mit dieser Vereinfachung, die bei vernünftiger Wahl der Gurtstäbe keinen grossen Einfluss auf die Genauigkeit der Lösung hat, wird die Berechnung wesentlich vereinfacht. 
Die Gurtstäbe bilden die Gurtungen des angenommenen räumlichen Fachwerkmodelles. Sie werden mit ungeraden Zahlen $i$ von 1 bis m bezeichnet, währenddem die zwischen den Gurtstäben liegenden Querschnittsteile mit geraden Zahlen $k$ von 2 bis $n$ numeriert werden.

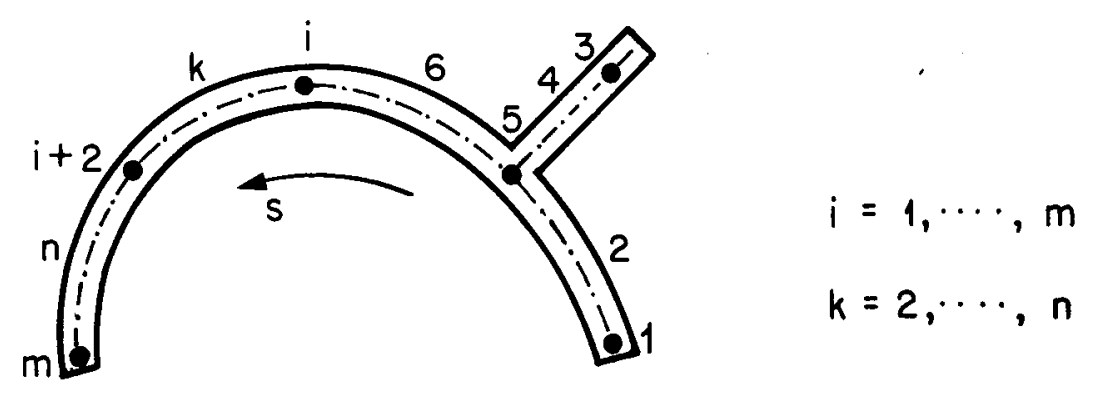

Bild 35

Die Querschnittsteile k übernehmen die Rolle von "Schubwänden", von denen jede einen konstanten Schubfluss $S_{k}$ aufweisen soll. Ist der Querschnitt wie in Bild 35 gekrümmt, so wird er durch einen polygonalen Querschnitt angenähert. Die ursprünglich gekrümmten "Schubwände" gehen dann in ebene Querschnittscheiben über, so dass für jede dieser Scheiben eine Schubresultierende $Q_{k}$ sowie der dazugehörende Hebelarm $\Gamma_{k}$ angegeben werden können.

Bei gekrümmtem Querschnitt weicht der effektive Hebelarm $r_{k}$ etwas vom $\mathrm{He}-$ belarm $\bar{r}_{k}$ der Bogensehne $f_{k}$ ab.

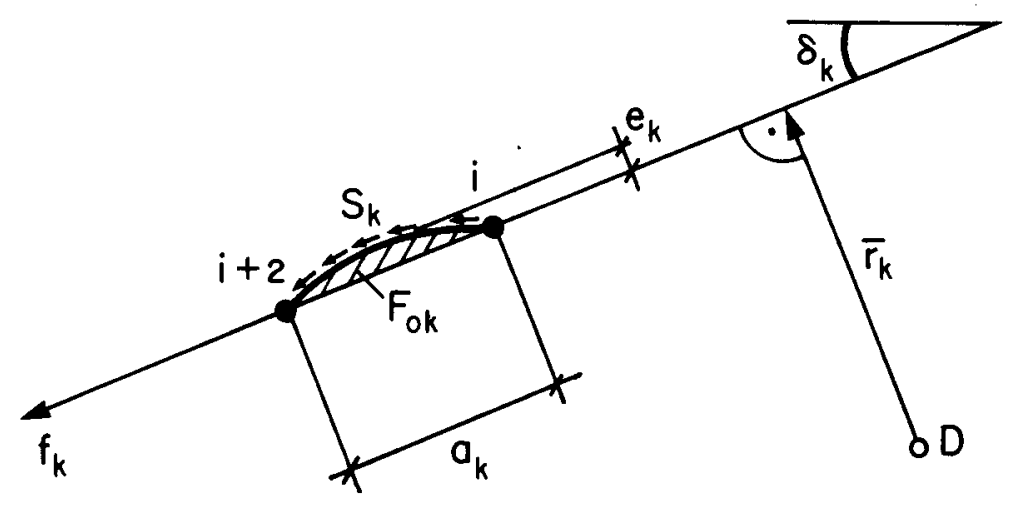

Bild 36

Wenn die Sehne $f_{k} z$ wischen zwei benachbarten Gurtstäben links des Querschnittbogens liegt (Blickrichtung: Positive s-Koordinate), so ist $F_{\text {ok }}$ bzw. $e_{k}$ positiv. 
Aus dem Anteil der k-ten "Schubwand" am Torsionsmoment $T_{w}$

$$
\begin{aligned}
\int_{i}^{i+2} s \cdot r \cdot d s & =s_{k} \cdot \int_{i}^{i+2} r \cdot d s=s_{k} \cdot\left(\bar{r}_{k} \cdot a_{k}+2 F_{o k}\right)= \\
& =\underbrace{s_{k} a_{k}}_{\substack{\text { Schubresul- } \\
\text { tierende }}} \cdot \underbrace{r_{k}}_{\begin{array}{l}
\text { Wirksamer } \\
\text { Hebelarm }
\end{array}}=Q_{k} \cdot r_{k}
\end{aligned}
$$

erhält man den gesuchten Hebelarm $\Gamma_{k}$ :

$$
I_{k}=\bar{r}_{k}+\frac{2 F_{o k}}{a_{k}}
$$

Dieser Ausdruck lässt sich noch vereinfachen, wenn man beachtet, dass der Querschnittsbogen zwischen zwei benachbarten Gurtstäben in der Regel ein flacher Bogen mit näherungsweise $F_{o k}=\frac{2}{3} \cdot a_{k} \cdot e_{k}$ ist.

$$
r_{k}=\bar{r}_{k}+\frac{4}{3} \cdot e_{k}
$$

Als Modell für die Wirkungsweise einer auf Schub beanspruchten Querschnittscheibe wird ein Fachwerk mit variabler Diagonalenneigung angenommen. In diesem Fachwerk bilden die Längseisen die Gurtungen, die Bügel die Pfosten und die Betondruckdiagonalen die Streben, wie dies Bild 37 zeigt.

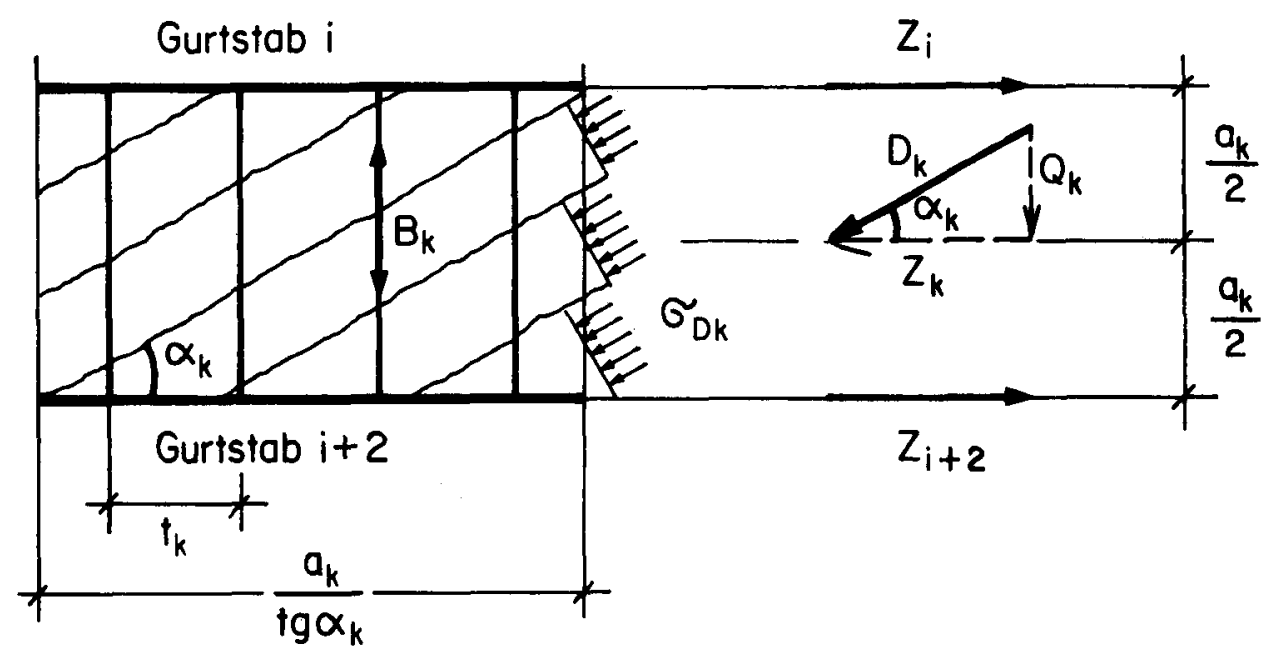


Die Annahme gleichmässig über die Querschnittscheibe verteilter Betondruckspannungen $\sigma_{D k}$, welche unter dem Winkel $\alpha_{k}$ zur Längsaxe geneigt sind, entspricht dem Ansatz eines konstanten Schubflusses für die Scheibe $k$. Die Komponenten dieser Betondruckspannungen in Längsrichtung sind ebenfalls gleichmässig über die Scheibe verteilt. Demzufolge resultiert aus der Schubbeanspruchung $Q_{k}$ eine in Mitte der Scheibe wirkende Längskraft $Z_{k}$ ' d.h., Längs- und Schubbeanspruchung beeinflussen einander. Die Vertikalkomponenten der Betondruckspannungen $\sigma_{D k}$ müssen mit den Bügelkräften $B_{k}$ im Gleichgewicht stehen. Dies ist nur möglich, wenn ein Ausbrechen der Druckdiagonalen durch einen engen Bügelabstand $t_{k}$ und/oder starke Gurtstäbe (in den Querschnittsecken und -enden) verhindert wird. Ist dies der Fall, so werden für die angenommene Spannungsverteilung die Bügel im dargestellten Scheibenabschnitt gleichmässig beansprucht, was zu folgenden Gleichgewichtsbedingungen führt:

$$
\begin{aligned}
& B_{k} \cdot \frac{a_{k}}{t_{k}} \cdot \frac{1}{\operatorname{tg} \alpha_{k}}=D_{k} \cdot \sin \alpha_{k} \\
& Q_{k}=D_{k} \cdot \sin \alpha_{k}=B_{k} \cdot \frac{a_{k}}{t_{k}} \cdot \frac{1}{\operatorname{tg} \alpha_{k}} \\
& z_{k}=D_{k} \cdot \cos \alpha_{k}=B_{k} \cdot \frac{a_{k}}{t_{k}} \cdot \frac{1}{\operatorname{tg}^{2} \alpha_{k}}
\end{aligned}
$$

In Uebereinstimmung mit der kinematischen Bedingung (22) wird für eine ebene Querschnittscheibe $k$ als Verschiebungsansatz eine konstante Schiebung $\gamma_{k}$ gewählt. Die Betondruckdiagonalen können als starr angesehen werden, da infolge der Beschränkung auf unterarmierte Querschnitte ein Versagen und damit für starrplastisches Materialverhalten eine Stauchung des Betons nicht zu erwarten ist. Aufgrund der Rissverzahnung kann sich eine Querschnittscheibe im allgemeinen nur senkrecht zu den Rissen bzw. senkrecht zu den Druckdiagonalen verformen. Dies führt bei einem konstanten Schiebungsansatz zu einem Verschiebungszustand, bei dem sich die Risse, die unter dem Winkel $\alpha_{k}$ zur Längsaxe geneigt sind, im Scheibenabschnitt $\frac{a_{k}}{\operatorname{tg} \alpha_{k}} \times a_{k}$ gleichmässig öffnen ( $g l$ leichmässige nominelle Rissdehnung $c_{k}$ ).

Danach vergrössern sich die Verschiebungen des Scheibenelementes proportional zum Abstand von der rissparallelen gestrichelten Geraden $g_{k}$, wie dies aus den Bildern 38 und 39 hervorgeht. Bild 38 gibt einen Ueberblick über die Abmessungen, währenddem in Bild 39 der Verschiebungszustand der k-ten Querschnittscheibe skizziert ist. 


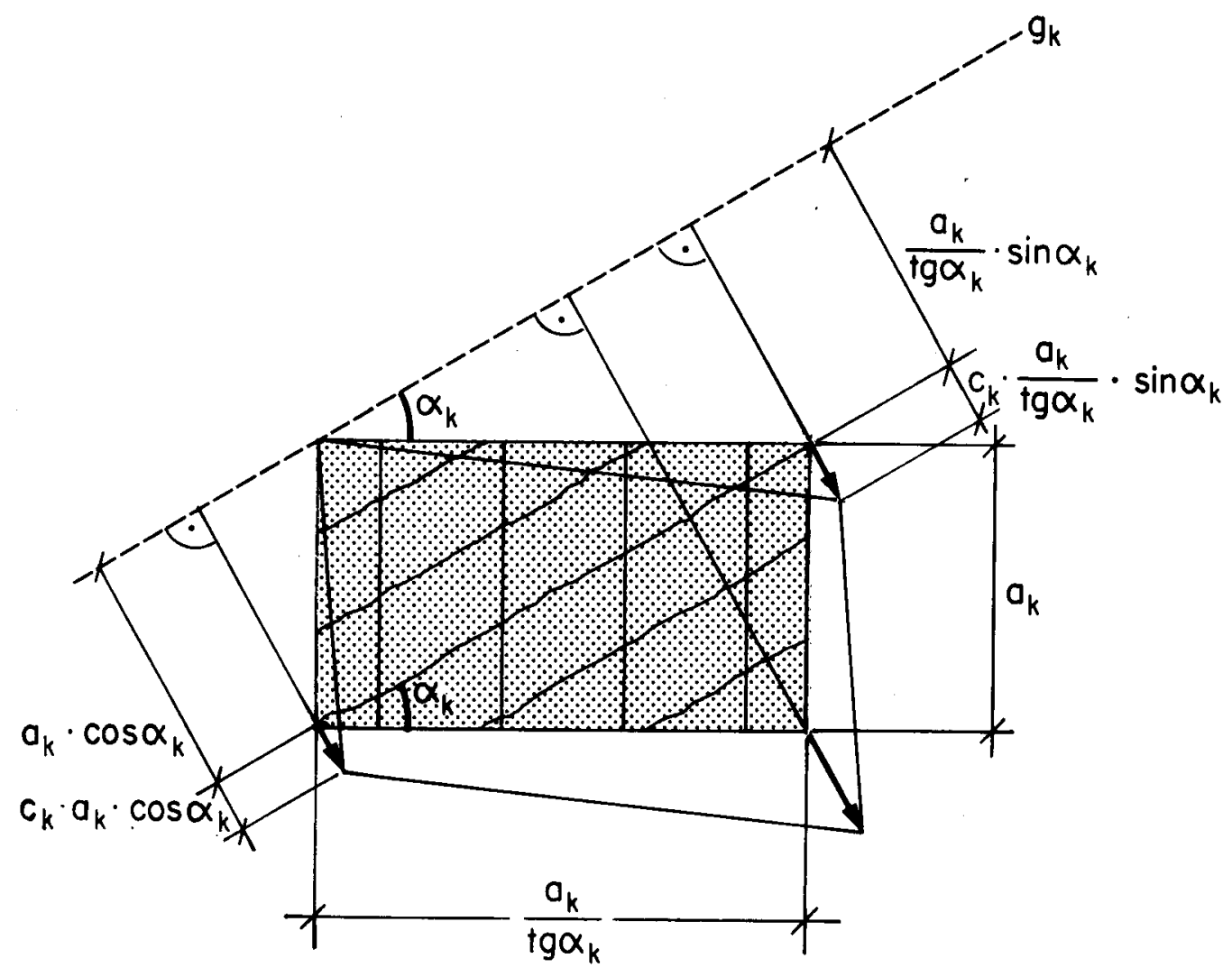

Bild 38

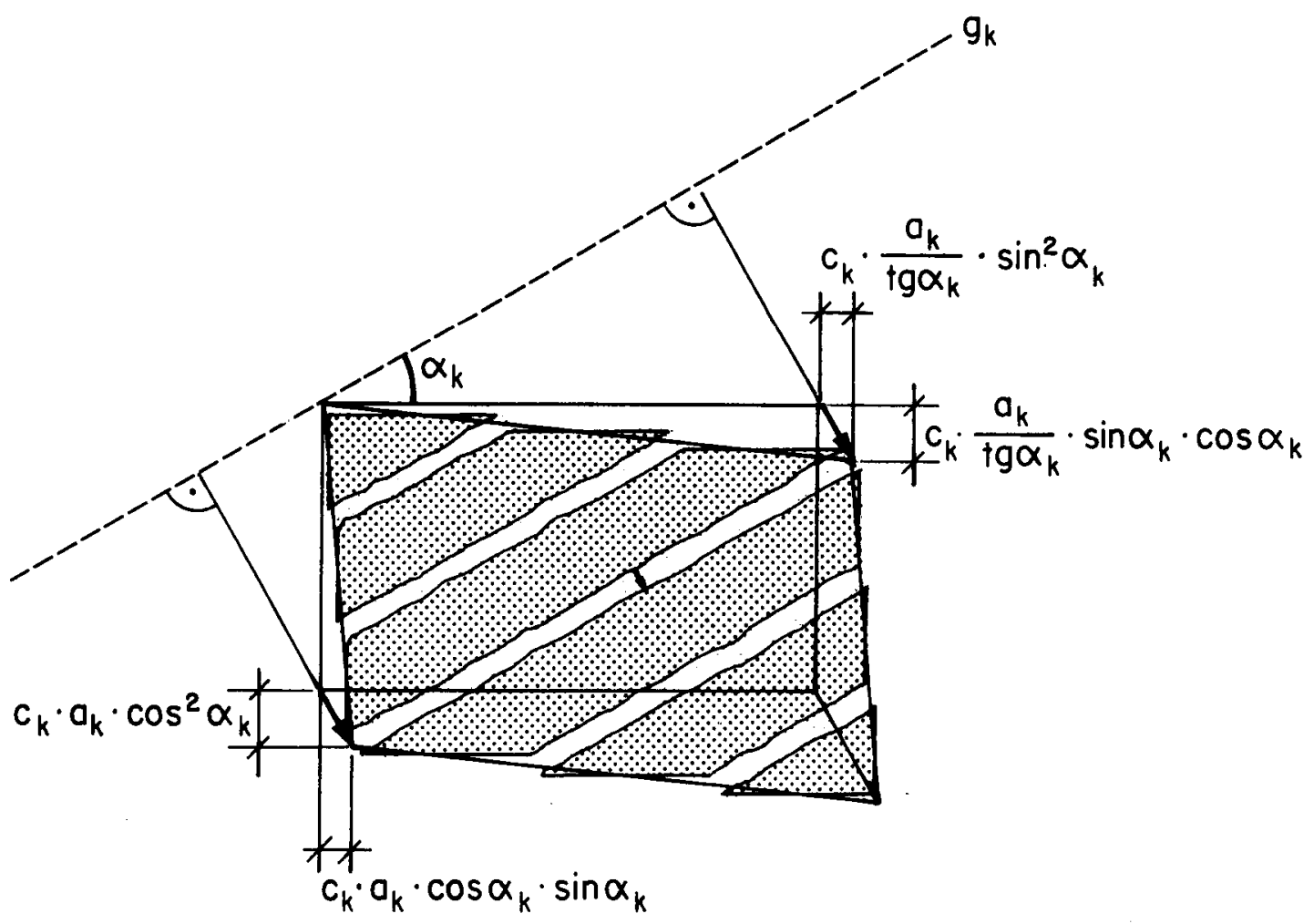


Entsprechend dem einfachen kinematischen Ansatz (konstante Schiebung) ist der Verschiebungszustand der Querschnittscheibe k durch den Verformungsparameter $c_{k}$ (gleichmässige nominelle Rissdehnung) gegeben. Die Längsdehnung $\varepsilon_{x k}$, die Bügeldehnung $\varepsilon_{B k}$ und die Schiebung $\gamma_{k}$ der k-ten Scheibe sind Funktionen dieses Verformungsparameters und können der dargestellten Verschiebungsfigur in Bild 39 entnommen werden.

$$
\begin{aligned}
& \varepsilon_{x k}=c_{k} \cdot \sin ^{2} \alpha_{k} \\
& \varepsilon_{B k}=c_{k} \cdot \cos ^{2} \alpha_{k} \\
& \gamma_{k}=c_{k} \cdot \sin \alpha_{k} \cdot \cos \alpha_{k}+c_{k} \cdot \cos \alpha_{k} \cdot \sin \alpha_{k}
\end{aligned}
$$

Anders geschrieben lauten diese kinematischen Bedingungen wie folgt:

$$
\begin{aligned}
& \varepsilon_{B k}=\varepsilon_{x k} \cdot \frac{1}{\operatorname{tg}^{2} \alpha_{k}} \\
& \gamma_{k}=\varepsilon_{x k} \cdot \frac{1}{\operatorname{tg} \alpha_{k}}+\varepsilon_{B k} \cdot \operatorname{tg} \alpha_{k} \\
& \text { und } \\
& c_{k}=\varepsilon_{x k}+\varepsilon_{B k}
\end{aligned}
$$

Nach der Beziehung (52b) wird die Schiebung $\gamma_{k}$ durch die Bügeldehnung $\varepsilon_{\text {Bk }}$ und durch die Längsdehnung $\varepsilon_{x k}$ bewirkt. Allerdings sind diese beiden Dehnungen gemäss (52a) miteinander gekoppelt, so dass die Schiebung als Funktion der Längsdehnung $\varepsilon_{x k}$ allein ausgedrückt werden kann.

$$
\gamma_{k}=\varepsilon_{x k} \cdot \frac{2}{t g \alpha_{k}}
$$

Der Ansatz einer konstanten Schiebung $\gamma_{k}$ führt für eine über die Scheibenhöhe $a_{k}$ durchgehend gleiche Diagonalenneigung $\alpha_{k} z u$ einer gleichmässigen Längsdehnung $\varepsilon_{x k}$, wie dies die Verschiebungsfigur des Bildes 39 zeigt. Normalerweise ist die Längsdehnung jedoch nicht konstant, sondern verläuft nach den im Abschnitt 2.3 gemachten Verschiebungsansätzen linear über die ebene Querschnittscheibe k. Für diesen Fall ergibt sich (bei konstanter Diagonalenneigung $\alpha_{k}$ ) auch eine lineare Verteilung der Schiebungen und der Bügeldehnungen. 

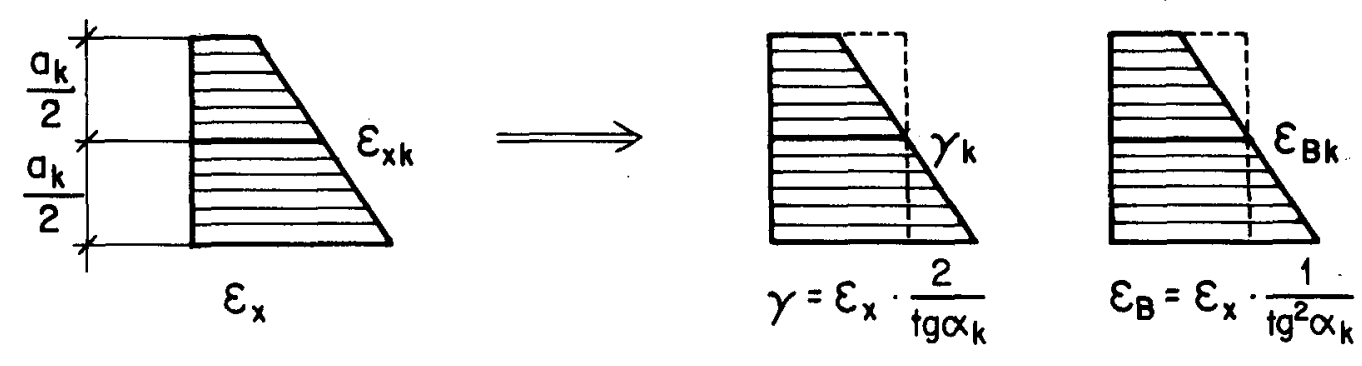

Bild 40

Wie sofort ersichtlich, ist die Dissipationsarbeit der Bügel über die Scheibenhöhe $a_{k}$ mit dem Mittelwert $\varepsilon_{B k}$ richtig erfasst. Hingegen ergibt sich aus der dargestellten linearen Schiebungsverteilung eine lokale Verletzung der kinematischen Bedingung (22), wonach die Schiebung einer ebenen Querschnittscheibe k konstant sein sollte. Betrachtet man den Mittelwert $\gamma_{k}$ als massgebend, so wird die kinematische Bedingung (22) im Mittel erfült. Da der Bruchwiderstand ebenfalls ein Mittelwert ist, kann erwartet werden, dass der mit den beschriebenen Ansätzen berechnete Bruchwiderstand nur wenig vom exakten Wert abweicht.

Folglich sind für eine Querschnittscheibe $k$ wie bei der statischen Betrachtung die Koordinaten in Scheibenmitte zu verwenden.

Die kinematischen Bedingungen zeigen, dass für den Mechanismus einer unterarmierten Querschnittscheibe im allgemeinen die Bügel und mindestens einer der beiden Gurtstäbe fliessen müssen. Diese Koppelung ist durch Gleichung (52a) ausgedrückt, welche besagt, dass sich die Risse senkrecht zu ihrer Neigung öffnen, was für kleine Rissöffnungen beim Eintreten des Mechanismus sicherlich zutrifft. Wie Bild 41 zeigt, vergrössern sich die beim Mechanismusbeginn (Fliessbeginn) vorhandenen Risse bzw. die nominelle Rissdehnung $c_{k}$ mit zunehmender Abweichung der Diagonalenneigung von $\left|\alpha_{k}\right|=45^{\circ}$.

Für Fliessbeginn Gurt: $\quad c_{k}=\varepsilon_{f} \cdot\left(1+\frac{1}{\operatorname{tg}^{2} \alpha_{k}}\right)$ Fliessbeginn Bügel: $\quad c_{k}=\varepsilon_{f} \cdot\left(1+\operatorname{tg}^{2} \alpha_{k}\right)$

Die kinematische Bedingung der orthogonalen Rissöffnung wird folglich mit zunehmender Abweichung der Diagonalenneigung von $\left|a_{k}\right|=45^{\circ}$ weniger zwingend, bis sich schliesslich die Risse beim Mechanismus derart öffnen, dass die Koppelung (52a) der Bügel- und Längsdehnungen wegfällt. 


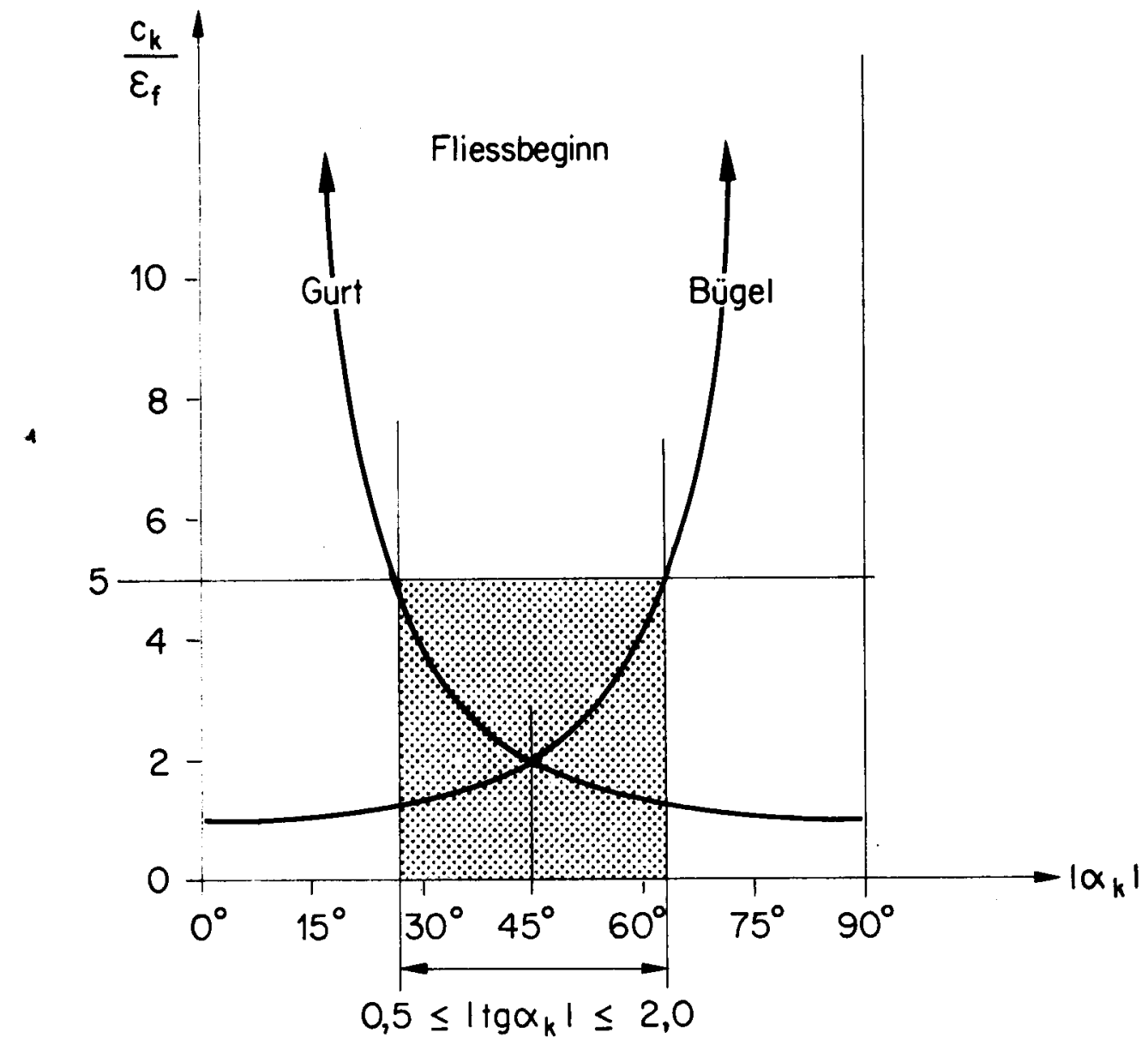

$c_{k}$ : nominelle Rissdehnung nach (52)

$\varepsilon_{f}$ : Stahldehnung bei Fliessbeginn

In schubbeanspruchten Querschnittscheiben dürfte dies ungefähr bei folgenden Diagonalenneigungen eintreten (vgl. Bild 41):
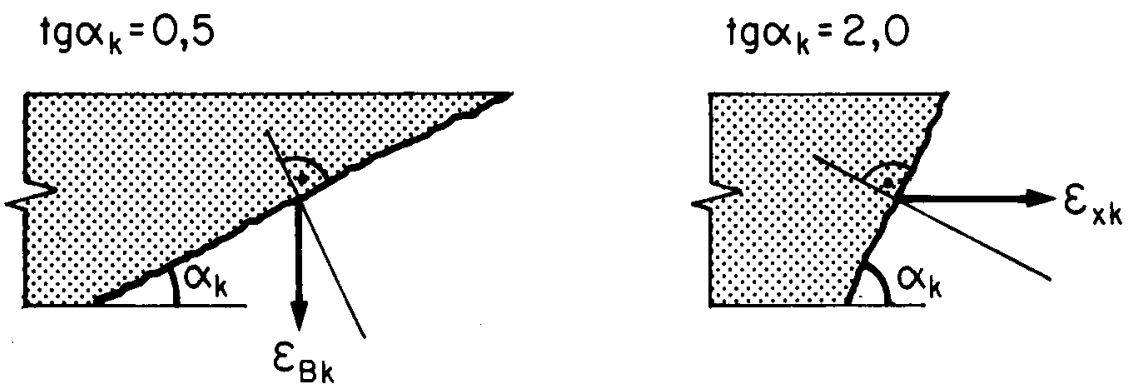
Für die dargestellten Grenzfälle führt das Fliessen der Bügel resp. eines Gurtstabes bereits zum Mechanismus, was eine weitere Umlagerung verunmöglicht. Die Diagonalenneigung schubbeanspruchter Querschnittscheiben lässt sich somit nicht beliebig zwischen $-90^{\circ}$ und $+90^{\circ}$ variieren. Sie bewegt sich nach Versuchsresultaten (siehe [9]) etwa in folgenden Grenzen:

$$
0,5 \leq\left|\operatorname{tg} \alpha_{k}\right| \leq 2,0
$$

Wie bei Stahlbeton- und Spannbetonquerschnitten üblich, wird ein eventuelles Mitwirken von Betonzugkräften vernachlässigt. Ausserdem ist, da nur unterarmierte Querschnitte untersucht werden, eine Betonzerstörung vor dem Fliessen der Armierung ausgeschlossen. Zur Berechnung des Bruchwiderstandes wird demnach für den Beton kein Materialgesetz benötigt. Die Fliessbedingungen für die Gurtstäbe und die Bügel lauten:

$$
\begin{aligned}
& z_{i}=Z_{f i} \\
& B_{k}=B_{f k} \\
& Z_{f i}: \text { Fliesskraft des Gurtstabes } i \\
& B_{f k}: \text { Fliesskraft eines Bügels der k-ten } \\
& \quad \text { Querschnittscheibe }
\end{aligned}
$$

Mit den Längsdehnungen $\varepsilon_{x i}$ der Gurtstäbe und den Bügeldehnungen $\varepsilon_{B k} 1$ ässt sich aus den obigen Beziehungen das Fliessgesetz für starr-idealplastisches Materialverhalten angeben.

$$
\begin{aligned}
& \varepsilon_{x i}>0 \rightarrow z_{i}=z_{f i} \\
& \varepsilon_{B k}>0 \rightarrow B_{k}=B_{f k}
\end{aligned}
$$

Die Bügeldehnungen $\varepsilon_{B k}$ sind dabei wie die Gurtstabdehnungen $\varepsilon_{x i}$ nichtnegative Variablen, da bei einem unterarmierten Querschnitt die Betondruckdiagonalen und die unter Druck stehenden Gurtstäbe als starr angesehen werden dürfen.

Sind einzelne Gurtstäbe oder die gesamte Zugarmierung vorgespannt, so ist die Vordehnung des Spannstahles bei der Berechnung des Bruchwiderstandes zu berücksichtigen. Bei Verbund der Spannarmierung mit dem Beton ergibt sich, falls die schlaffe und die Spannarmierung die Fliess-, resp. Streck- 
grenze bei ähnlichen zusätzlichen Dehnungen erreichen, kein Unterschied im Bruchverhalten von unterarmierten Querschnitten. Demnach können bei normalen Armierungsgehalten vorgespannte Tragwerke genau gleich wie nicht vorgespannte behandelt werden. Die grundlegenden Beziehungen (51) bis (55) ermöglichen es, die Berechnung von unterarmierten Stahlbeton- und Spannbetonquerschnitten in Angriff zu nehmen. Dabei wird wie im vierten Kapitel sowohl nach dem statischen als auch nach dem kinematischen Grenzwertsatz der Plastizitätstheorie vorgegangen.

\subsection{Statische Methode}

Unter Einhaltung der Gleichgewichts- und Plastizitätsbedingungen wird jene Beanspruchung $\lambda \cdot\left(N, M_{y}, M_{z}, M_{w}, Q_{y}, Q_{z}, T_{w}\right)$ gesucht, für die der Widerstandsfaktor $\lambda$ maximal wird. Dabei wird eine proportionale Steigerung der Beanspruchung angenommen.

\section{Gleichgewichtsbedingungen:}

Die Gleichgewichtsbedingungen (15) lauten mit dem Widerstandsfaktor $\lambda$ sowie den Gurtstabkräften $Z_{i}$, den Schubresultierenden $Q_{k}$ der einzelnen Querschnittscheiben und den daraus resultierenden Längskräften $Z_{k}$ folgendermassen:

$$
\begin{aligned}
& 0=-\lambda \cdot N+\sum_{i=1}^{m} z_{i}-\sum_{k=2}^{n} z_{k} \\
& 0=-\lambda \cdot M_{y}+\sum_{i=1}^{m} z_{i} \cdot y_{i}-\sum_{k=2}^{n} z_{k} \cdot y_{k} \\
& 0=-\lambda \cdot M_{z}+\sum_{i=1}^{m} z_{i} \cdot z_{i}-\sum_{k=2}^{n} z_{k} \cdot z_{k} \\
& 0=-\lambda \cdot M_{\omega}+\sum_{i=1}^{m} z_{i} \cdot \omega_{i}-\sum_{k=2}^{n} z_{k} \cdot \omega_{k} \\
& 0=-\lambda \cdot Q_{y}+\sum_{k=2}^{n} Q_{k} \cdot \cos \delta_{k} \\
& 0=-\lambda \cdot Q_{z}+\sum_{k=2}^{n} Q_{k} \cdot \sin \delta_{k} \\
& 0=-\lambda \cdot T_{w}+\sum_{k=2}^{n} Q_{k} \cdot r_{k}
\end{aligned}
$$

Die in diesen Gleichungen vorkommenden geometrischen Grössen $y_{k}, z_{k}$ und $\omega_{k}$ beziehen sich jeweils auf die Mitte der einzelnen Querschnittscheiben. Bei 
gekrümmtem Querschnitt wird als Winkel $\delta_{k}$ der Winkel zwischen der Scheibensehne $f_{k}$ und der Koordinatenaxe y eingesetzt, wie dies Bild 36 zeigt. Der wirksame Hebelarm $r_{k}$ der k-ten Querschnittscheibe kann in diesem Fail der Gleichung (50) entnammen werden.

Bei Verwendung der Beziehungen (51) können die Schubkräfte $Q_{k}$ und die Längskräfte $Z_{k}$ durch die Bügelkräfte $B_{k}$ ausgedrückt werden. Die Gleichgewichtsbedingungen (56) transformieren sich damit zu:

$$
\begin{aligned}
& 0=-\lambda \cdot N+\sum_{i=1}^{m} z_{i} \quad-\sum_{k=2}^{n} B_{k} \cdot \frac{a_{k}}{t_{k}} \cdot \frac{1}{\operatorname{tg}^{2} \alpha_{k}} \\
& 0=-\lambda \cdot M_{y}+\sum_{i=1}^{m} z_{i} \cdot y_{i}-\sum_{k=2}^{n} B_{k} \cdot \frac{a_{k}}{t_{k}} \cdot \frac{y_{k}}{\operatorname{tg}^{2} \alpha_{k}} \\
& 0=-\lambda \cdot M_{z}+\sum_{i=1}^{m} z_{i} \cdot z_{i}-\sum_{k=2}^{n} B_{k} \cdot \frac{a_{k}}{t_{k}} \cdot \frac{z_{k}}{\operatorname{tg}^{2} \alpha_{k}} \\
& 0=-\lambda \cdot M_{w}+\sum_{i=1}^{m} z_{i} \cdot \omega_{i}-\sum_{k=2}^{n} B_{k} \cdot \frac{a_{k}}{t_{k}} \cdot \frac{\omega_{k}}{\operatorname{tg}^{2} \alpha_{k}} \\
& 0=-\lambda \cdot Q_{y}+\sum_{k=2}^{n} B_{k} \cdot \frac{a_{k}}{t_{k}} \cdot \frac{\cos \delta_{k}}{\operatorname{tg} \alpha_{k}} \\
& 0=-\lambda \cdot Q_{z}+\sum_{k=2}^{n} B_{k} \cdot \frac{a_{k}}{t_{k}} \cdot \frac{\sin \delta_{k}}{\operatorname{tg} \alpha_{k}} \\
& 0=-\lambda \cdot T_{w}+\sum_{k=2}^{n} B_{k} \cdot \frac{a_{k}}{t_{k}} \cdot \frac{r_{k}}{\operatorname{tg} \alpha_{k}}
\end{aligned}
$$

\section{Plastizitätsbedingungen:}

Die Plastizitätsbedingungen verlangen, dass die Gurtstab- und die Bügelkräfte ihre Fliesskräfte nicht übersteigen.

$$
\begin{aligned}
& z_{i} \leq z_{f i} \\
& B_{k} \leq B_{f k}
\end{aligned}
$$


Mit Einführung nichtnegativer Schlupfvariablen $s_{i}$ bzw. $s_{k} k o ̈ n n e n ~ d i e s e$ Ungleichungen (58) in folgender Form angeschrieben werden.

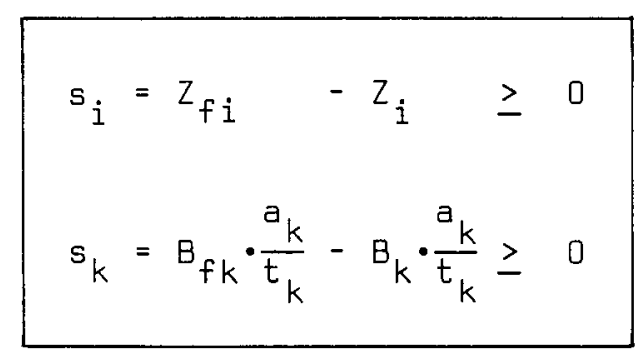

Das Weglassen der Plastizitätsbedingungen für den Beton ist gleichbedeutend mit der Annahme, dass der Beton vorläufig unbeschränkte Druckkräfte aufnehmen kann. Die Bestimmung des Querschnittswiderstandes erfolgt mit dieser für unterarmierte Querschnitte hinreichenden Voraussetzung.

Selbstverständlich ist nach erfolgter Berechnung zu kontrollieren, dass die unter Druck stehenden Gurtstäbe und die Druckdiagonalen ihre Bruchwiderstände tatsächlich nicht erreichen (siehe Abschnitt 5.7).

\section{Optimierung:}

Werden die Gleichgewichtsbedingungen (57) und die Plastizitätsbedingungen (59) in Tableauform zusammengestellt, so erhält man das Programm für den zu maximierenden Widerstandsfaktor $\lambda$. Dieses Programm ist nichtlinear, da in den Restriktionen (57) die variablen Diagonalenneigungen $\alpha_{k}$ in der form von $\operatorname{tg}^{2} \alpha_{k}$ bzw. tga $\alpha_{k}$ zusammen mit den unbekannten Bügelkräften $B_{k}$ vorkammen.

Für die numerische Auflösung dieses nichtlinearen Programmes fehlen bis heute die mathematischen Grundlagen. Aus diesem Grunde muss eine Näherungslösung gesucht werden. Eine einfache Methode zur Bestimmung einer für praktische Zwecke ausreichenden unteren Schranke des Querschnittswiderstandes wird in Abschnitt 5.6 angegeben. 
unbeschränkte Variablen nichłnegative Variablen

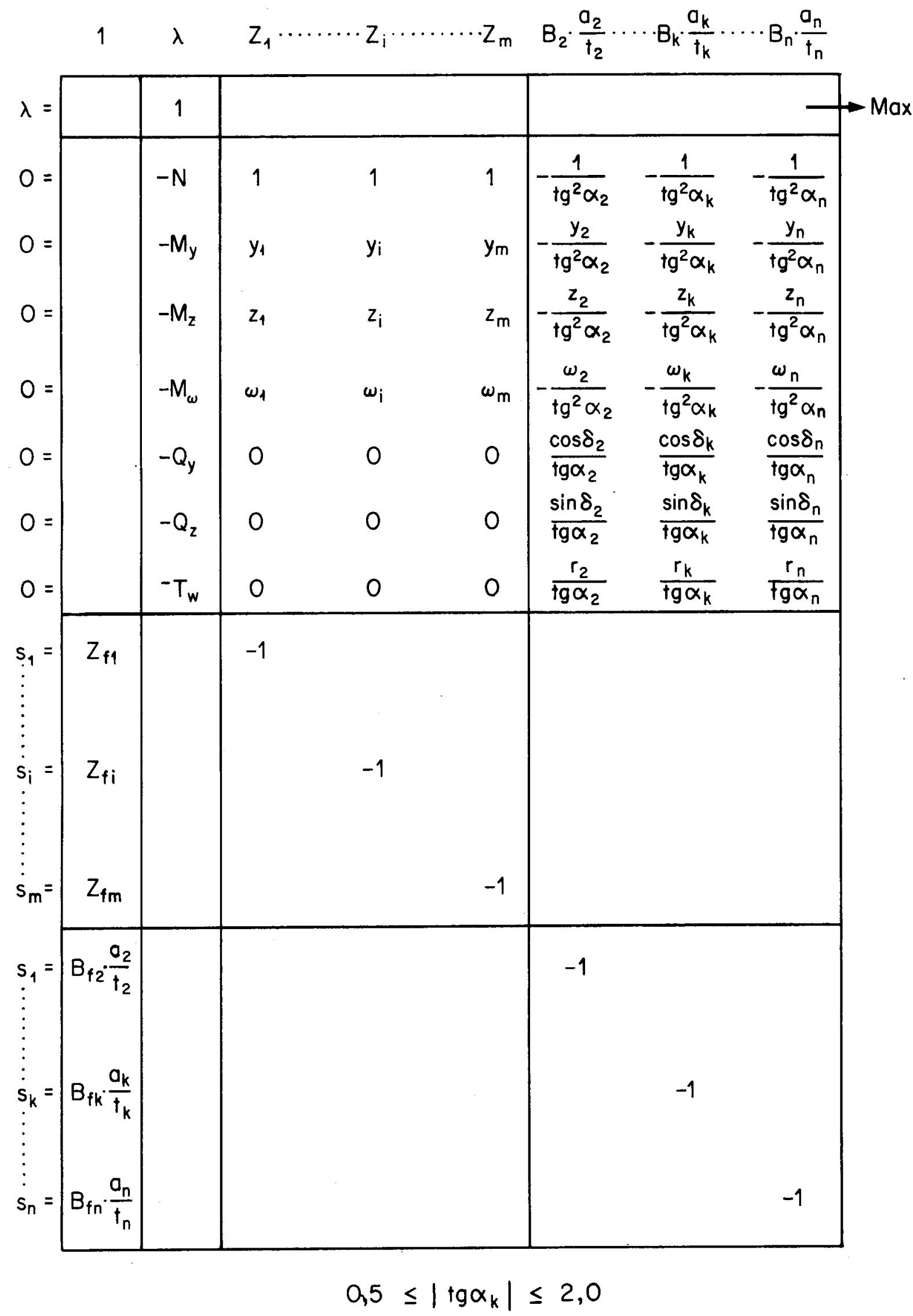


Unter Annahme proportionaler Steigerung der Beanspruchung $\lambda \cdot\left(N, M_{y}, M_{z}\right.$, $M_{w}$, $Q_{y}, Q_{z}, T_{w}$ ' wird jener Querschnitts-Mechanismus gesucht, für den die geleistete Arbeit verschwindet und der Widerstandsfaktor $\lambda$ minimal wird.

\section{Mechanismusbedingungen:}

Nach der im zweiten Kapitel hergeleiteten kinematischen Bedingung (19) lautet die Mechanismusbedingung für den Gurtstab i:

$$
\varepsilon_{x i}=u^{\prime}-v^{\prime \prime} \cdot y_{i}-w^{\prime \prime} \cdot z_{i}-\vartheta " \cdot \omega_{i}
$$

Ferner werden die aus (52) übernommenen kinematischen Bedingungen für die Querschnittscheibe $k$ benötigt.

$$
\begin{aligned}
& \varepsilon_{B k}=\gamma_{k} \cdot \frac{1}{t g \alpha_{k}}-\varepsilon_{x k} \cdot \frac{1}{t^{2} \alpha_{k}} \\
& \varepsilon_{B k}=\varepsilon_{x k} \cdot \frac{1}{t^{2} \alpha_{k}}
\end{aligned}
$$

Diese Gleichungen führen mit den für die Scheibe $k$ formulierten kinematischen Bedingungen (19) und (22)

$$
\begin{aligned}
& \varepsilon_{x k}=u^{\prime}-v^{\prime \prime} \cdot y_{k}-w^{\prime \prime} \cdot z_{k}-\vartheta " \cdot \omega_{k} \\
& \gamma_{k}=\gamma_{y} \cdot \cos \delta_{k}+\gamma_{z} \cdot \sin \delta_{k}+\gamma_{\vartheta} \cdot r_{k}
\end{aligned}
$$

zu folgenden Mechanismusbedingungen:

$$
\begin{aligned}
\varepsilon_{B k}= & \frac{1}{\operatorname{tg} \alpha_{k}} \cdot\left(\gamma_{y} \cdot \cos \delta_{k}+\gamma_{z} \cdot \sin \delta_{k}+\gamma_{\vartheta} \cdot r_{k}\right)- \\
& -\frac{1}{\operatorname{tg}^{2} \alpha_{k}} \cdot\left(u^{\prime}-v^{\prime \prime} \cdot y_{k}-w^{\prime \prime} \cdot z_{k}-\vartheta " \cdot \omega_{k}\right) \\
\varepsilon_{B k} & =\frac{1}{\operatorname{tg}^{2} \alpha_{k}} \cdot\left(u^{\prime}-v^{\prime \prime} \cdot y_{k}-w^{\prime \prime} \cdot z_{k}-\vartheta " \cdot \omega_{k}\right)
\end{aligned}
$$




\section{Arbeitsbedingung:}

Die Arbeitsbedingung, nach welcher die beim Mechanismus geleistete Arbeit verschwinden muss, wird hier am Stabelement der Länge $\Delta x=1$ formuliert.

$$
A_{a}+A_{i}=0
$$

oder

$$
A_{a}=-A_{i}=A_{d}
$$

$A_{a}:$ Arbeit der äusseren kräfte (hier: Schnittkräfte)

$A_{d}$ : Dissipationsarbeit, d.h. negative Arbeit der inneren Kräfte (Spannungen)

Die Arbeit der äusseren Kräfte für den Verschiebungszustand $u^{\prime}, v^{\prime \prime}, w^{\prime \prime}, \vartheta "$, $\gamma_{y}, \gamma_{z}$ und $\gamma_{\vartheta}$ kann der Gleichung (45) entnommen werden.

$$
\begin{aligned}
A_{a}=\lambda \cdot\left[u^{\prime} \cdot N-v " \cdot M_{y}-w " \cdot M_{z}-\vartheta " \cdot M_{w}+\right. \\
\left.+\gamma_{y} \cdot Q_{y}+\gamma_{z} \cdot Q_{z}+\gamma_{\vartheta} \cdot T_{w}\right]
\end{aligned}
$$

Infolge der Beschränkung auf unterarmierte Querschnitte leisten nur die Gurtstäbe und die Bügel einen Beitrag zur Dissipationsarbeit,

$$
A_{d}=\sum_{i=1}^{m} Z_{i} \cdot \varepsilon_{x i}+\sum_{k=2}^{n} B_{k} \cdot \frac{a_{k}}{t_{k}} \cdot \varepsilon_{B k}
$$

welche sich mit Hilfe des Fliessgesetzes (55) wie folgt umformen lässt.

$$
A_{d}=\sum_{i=1}^{m} Z_{f i} \cdot \varepsilon_{x i}+\sum_{k=2}^{n} B_{f k} \cdot \frac{a_{k}}{t_{k}} \cdot \varepsilon_{B k}
$$

Damit erhält man aus der Arbeitsbedingung $A_{a}=A_{d}$ den zu minimierenden Widerstandsfaktor $\lambda$.

$$
\lambda=\frac{\sum_{i=1}^{m} z_{f i} \cdot \varepsilon_{x i}+\sum_{k=2}^{n} B_{f k} \cdot \frac{a_{k}}{t_{k}} \cdot \varepsilon_{B k}}{u^{\prime} \cdot N-\ldots \ldots+\cdots+\gamma_{\vartheta} \cdot T_{w}}
$$


Er kann schliesslich, da der absolute Betrag der Verformungen keine Rolle spielt, aus dem Zähler des obigen Ausdruckes

$$
\lambda=\sum_{i=1}^{m} z_{f i} \cdot \varepsilon_{x i}+\sum_{k=2}^{n} B_{f k} \cdot \frac{a_{k}}{t_{k}} \cdot \varepsilon_{B k}
$$

mit dem Nenner als Nebenbedingung bestimmt werden.

$$
u^{\prime} \cdot N-v^{\prime \prime} \cdot M_{y}-w^{\prime \prime} \cdot M_{z}-\vartheta " \cdot M_{w}+\gamma_{y} \cdot Q_{y}+\gamma_{z} \cdot Q_{z}+\gamma_{\vartheta} \cdot T_{w}=1
$$

\section{Optimierung:}

Die Gleichungen (60), (62), (65) und (66) können in Tableauform zusammengestellt werden, womit man das Programm für den zu minimierenden Widerstandsfaktor $\lambda$ erhält.

Dieses Programm ist genauso wie dasjenige der statischen Methode in Bild 43 nichtlinear. Vergleicht man die beiden Optimierungsprogramme, so stellt man fest, dass die aus dem Teil A der Minimumsaufgabe (Bild 44) gebildete Koeffizientenmatrix mit der transponierten Koeffizientenmatrix der Maximumsaufgabe (Bild 43) identisch ist. Im Teil B der Minimumsaufgabe erscheinen zusätzlich Restriktionen, die die Orthogonalität der Rissöffnungen beschreiben. Auf den ersten Blick ist eine Dualität zwischen den beiden Programmen nicht zu erkennen. Dies erstaunt bei den vorliegenden Programmen keineswegs, da sie nichtlinear und die Nichtlinearitäten (z.B. tga ) ausserdem nichtkonvex sind. Die zentrale Frage, ob beide Programme zu ein und demselben Widerstandsfaktor $\lambda$ führen, wird im nächsten Abschnitt beantwortet. 


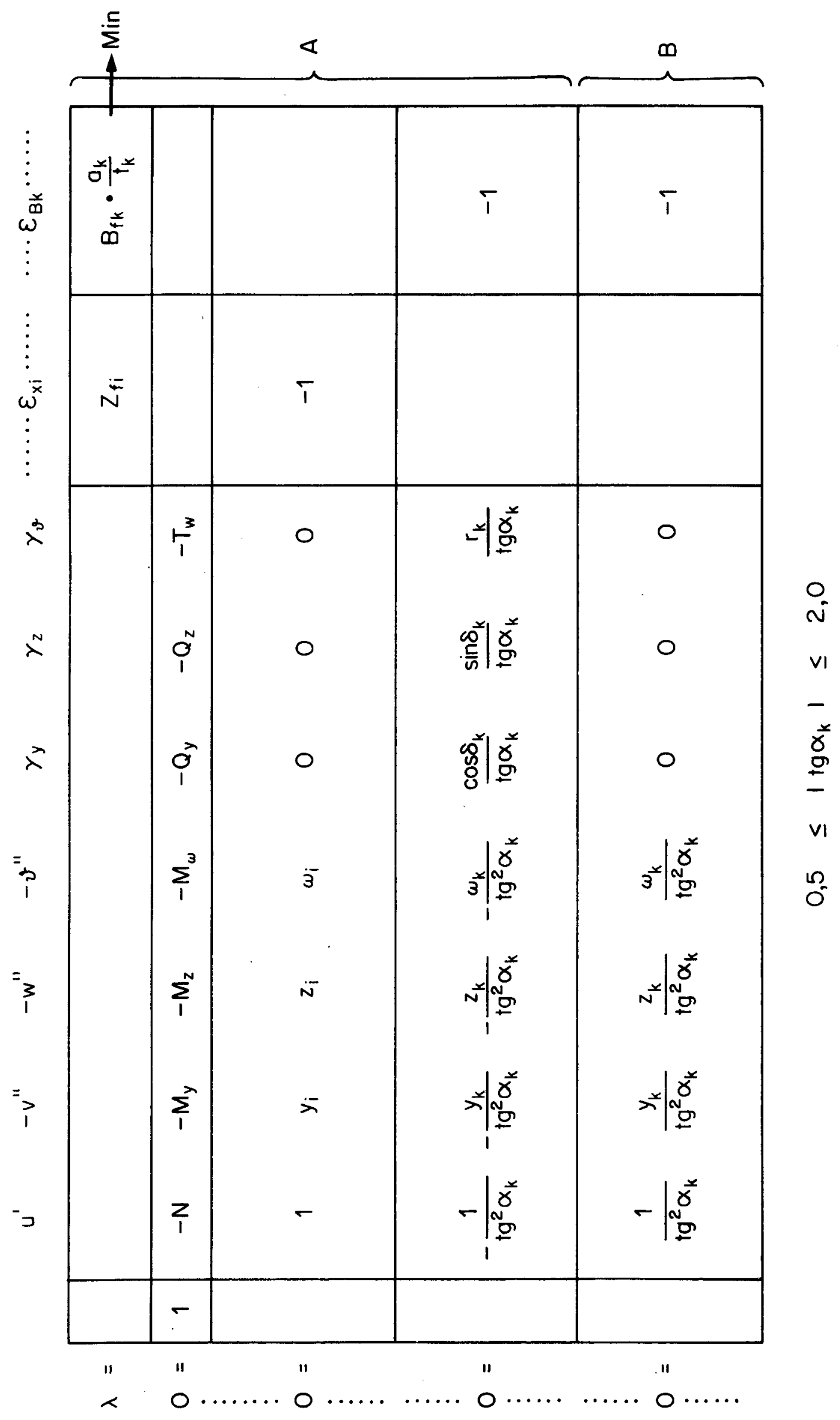


Um zu zeigen, dass die Programme der statischen und der kinematischen Methode dual sind, d.h., dass beide Programme denselben Widerstandsfaktor $\lambda$ liefern, genügt die Betrachtung einer einzelnen Querschnittscheibe, denn die gesamte Nichtlinearität ist bereits im angenommenen Fachwerkmodell in Form der variablen Diagonalenneigung enthalten.

Im folgenden wird eine Querschnittscheibe untersucht, deren Gurtstäbe die Fliesskräfte $Z_{f o} b z w . Z_{f u}$ und deren Bügel die Fliesskräfte $B_{f}$ aufweisen. Sie wird, wie dies aus Bild 45 hervorgeht, durch eine Querkraft $Q$ und eine in Scheibenmitte angreifende Normalkraft $N$ beansprucht. Die Wirkungsweise der Querschnittscheibe wird durch ein Fachwerkmodell mit variabler Diagonalenneigung beschrieben. Es gelten demnach die in Abschnitt 5.1 hergeleiteten statischen und kinematischen Beziehungen.
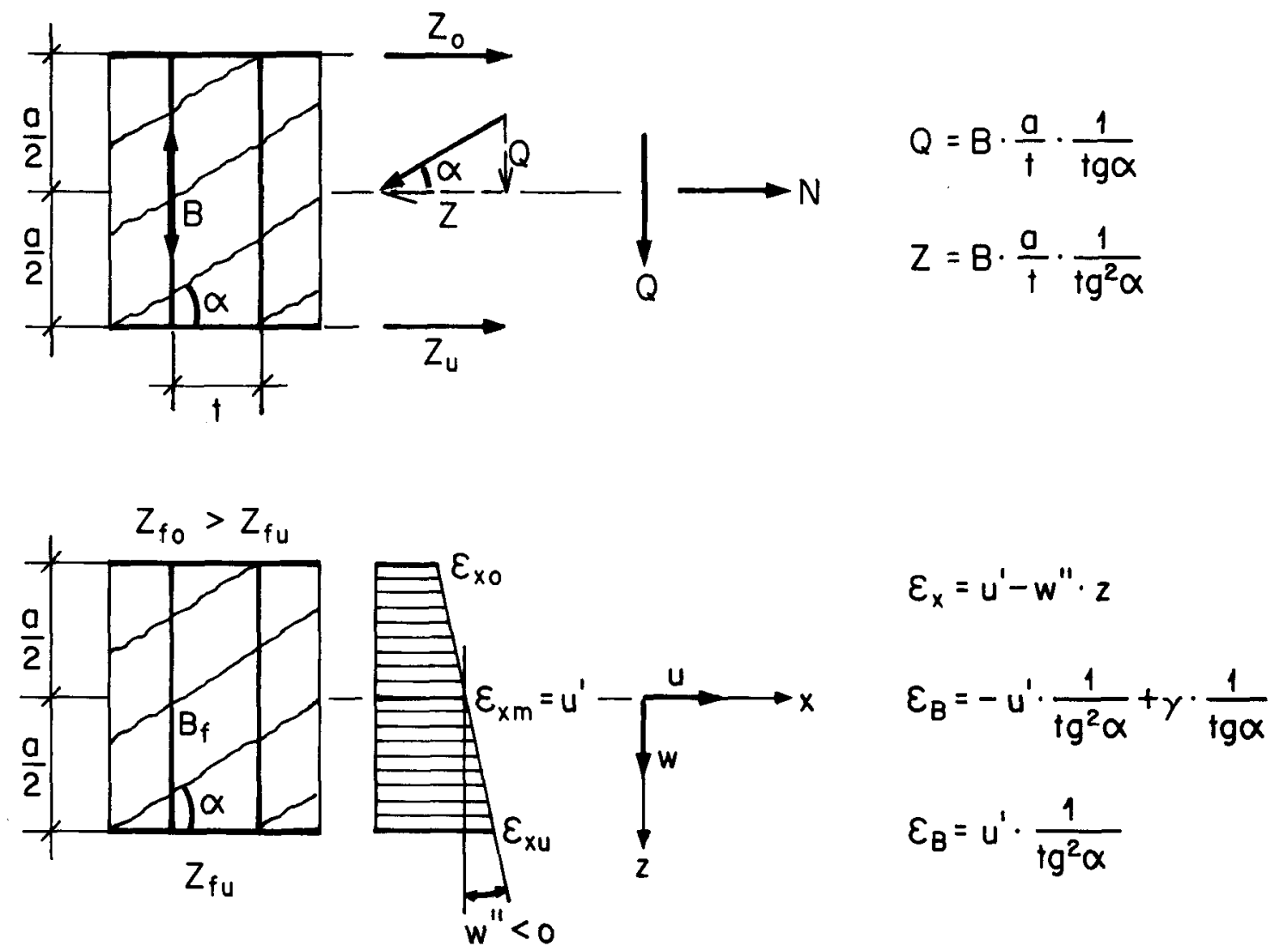

Bild 45

Das Gleichgewicht kann für den dargestellten einfachen Fall durch drei Bedingungen, nämlich durch je eine Gleichung für die Querkraft $Q$, die Normalkraft $N$ und das Biegemoment $M=0$ formuliert werden. Der Verschiebungszustand ist durch die drei Parameter $u^{\prime},{ }^{\prime \prime}$ " und $\gamma$ gegeben. 
Bei genau gleichem Vorgehen wie in den beiden vorangegangenen Abschnitten liefern die statische und die kinematische Methode folgende nichtlinearen Programme zur Bestimmung des Widerstandsfaktors $\lambda$.

Maximumsaufgabe (statische Methode)

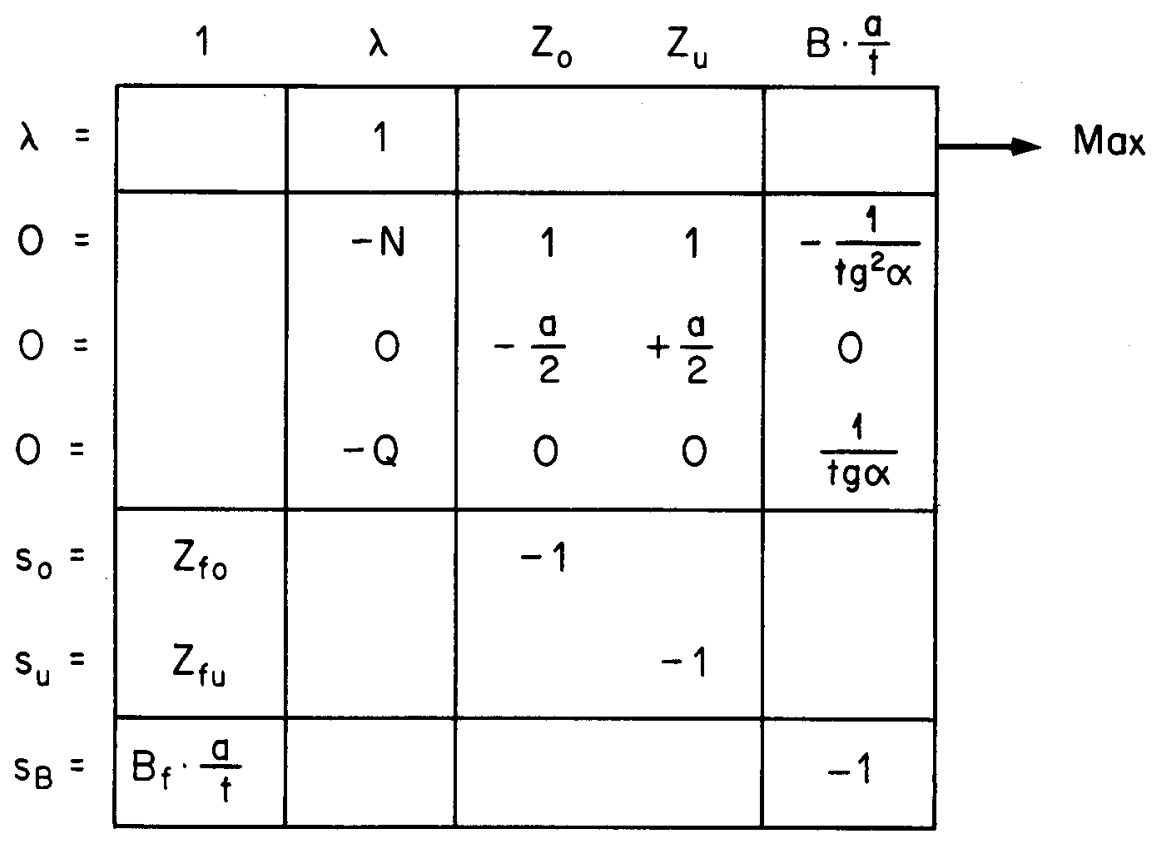

Minimumsaufgabe (kinematische Methode)

\begin{tabular}{|c|c|c|c|c|c|c|c|}
\hline & 1 & $u^{\prime}$ & $-w^{\prime \prime}$ & $\gamma$ & $\varepsilon_{x 0}$ & $\varepsilon_{x u}$ & $\varepsilon_{B}$ \\
\hline$=$ & & & & & $Z_{f 0}$ & $Z_{f u}$ & $B_{f} \cdot \frac{a}{t}$ \\
\hline $0=$ & 1 & $-N$ & 0 & $-Q$ & & & \\
\hline $0=$ & & 1 & $-\frac{a}{2}$ & 0 & -1 & & \\
\hline $0=$ & & 1 & $+\frac{a}{2}$ & 0 & & -1 & \\
\hline $0=$ & & $-\frac{1}{\operatorname{tg}^{2} \alpha}$ & 0 & $\frac{1}{\operatorname{tg} \alpha}$ & & & -1 \\
\hline$=$ & & $\frac{1}{\operatorname{tg}^{2} \alpha}$ & 0 & 0 & & & -1 \\
\hline
\end{tabular}


Die hier angegebenen einfachen Programme weisen denselben Aufbau und dieselben nichtlinearen Besonderheiten auf wie die in den Abschnitten 5.2 und 5.3 hergeleiteten Programme, so dass eine Dualität zwischen den beiden nichtlinearen Programmen der Bilder 46 und 47 auch auf die früher aufgestellten Programme für offene stahlbetonquerschnitte übertragen werden kann. Somit braucht man nur noch zu zeigen, dass für das hier betrachtete einfache Beispiel sowohl die Maximumsaufgabe als auch die Minimumsaufgabe zum selben Widerstandsfaktor $\lambda$ führen.

\section{Lösung der Maximumsaufgabe:}

Setzt man die Momentenbedingung, nach der die Gurtstabkräfte $z_{0}$ und $z_{u}$ gleich gross sein müssen, in die übrigen Gleichgewichtsbedingungen ein, so lauten diese:

$$
\begin{aligned}
& \lambda \cdot N=Z \cdot Z_{U}-B \cdot \frac{a}{t} \cdot \frac{1}{\operatorname{tg}^{2} \alpha} \\
& \dot{\lambda} \cdot Q=B \cdot \frac{a}{t} \cdot \frac{1}{\operatorname{tg} \alpha}
\end{aligned}
$$

Wie leicht einzusehen ist, wird das Maximum des Widerstandsfaktors $\lambda$ dann erreicht, wenn unter Einhaltung der Gleichgewichts- und Plastizitätsbedingungen ein möglichst grosser Anteil der Armierung ins Fliessen kommt. Dies ist hier der Fall, wenn die Bügel und der untere Gurtstab fliessen.

$$
\begin{aligned}
& \lambda_{\text {max }} \cdot N=2 \cdot Z_{f u}-B_{f} \cdot \frac{a}{t} \cdot \frac{1}{\operatorname{tg}^{2} \alpha} \\
& \lambda_{\text {max }} \cdot Q=B_{f} \cdot \frac{a}{t} \cdot \frac{1}{\operatorname{tg} \alpha}
\end{aligned}
$$

Für das vorausgesetzte Armierungsverhältnis $Z_{f o}>Z_{f u}$ darf die obere Gurtstabkraft $Z_{0}$ ihre Fliesskraft nicht erreichen, da sonst (mit der Momentenbedingung $z_{0}=z_{u}$ ) die Plastizitätsbedingung $z_{u} \leq z_{f u}$ für den unteren Gurtstab nicht mehr erfüllt wäre.

Aus den beiden Gleichungen (67) lassen sich die Diagonalenneigung und der Widerstandsfaktor bestimmen. Eliminiert man $\lambda_{\max }$ aus den Beziehungen (67), so erhält man folgende quadratische Gleichung für die Diagonalenneigung $\alpha$ bzw. für die Funktion $\frac{1}{\operatorname{tg} \alpha}$.

$$
\frac{1}{\operatorname{tg}^{2} \alpha}+\frac{N}{Q} \cdot \frac{1}{\operatorname{tg} \alpha}-\frac{2 \cdot Z_{f u}}{B_{f} \cdot \frac{a}{t}}=0
$$


Eine Beziehung für $\lambda_{\max }$ ergibt sich bei Elimination der Diagonalenneigung,

$$
\lambda_{\max }^{2}+\frac{N \cdot B_{f} \cdot \frac{a}{t}}{Q^{2}} \cdot \lambda_{\max }-\frac{2 \cdot Z_{f u} \cdot B_{f} \cdot \frac{a}{t}}{Q^{2}}=0
$$

womit die Maximumsaufgabe gelöst ist.

$$
\lambda_{\max }=\frac{N \cdot B_{f} \cdot \frac{a}{t}}{2 \cdot Q^{2}} \cdot\left[-1+\sqrt{1+\frac{8 \cdot Z f u}{B_{f} \cdot \frac{a}{t}} \cdot\left(\frac{Q}{N}\right)^{2}}\right]
$$

\section{Lösung der Minimumsaufgabe:}

Der gesamte Verschiebungszustand kann durch die Gurtstabdehnungen $\varepsilon_{x o}$ und $\varepsilon_{x u}$ dargestellt werden, wie dies aus Bild 45 oder aus den Restriktionen der Minimumsaufgabe (Bild 47) hervorgeht. Es gilt:

$$
\begin{aligned}
u^{\prime} & =\frac{1}{2} \cdot\left(\varepsilon_{x u}+\varepsilon_{x O}\right) \\
-w^{\prime} & =\frac{1}{a} \cdot\left(\varepsilon_{x u}-\varepsilon_{x O}\right) \\
\gamma & =\frac{1}{\operatorname{tg} \alpha} \cdot\left(\varepsilon_{x u}+\varepsilon_{x O}\right) \\
\varepsilon_{B} & =\frac{1}{2 \cdot \operatorname{tg}^{2} \alpha} \cdot\left(\varepsilon_{x u}+\varepsilon_{x O}\right)
\end{aligned}
$$

Mit diesen kinematischen Bedingungen transformiert sich die in den ersten zwei Zeilen der Minimumsaufgabe (Bild 47) enthaltene Beziehung für den Widerstandsfaktor $\lambda$

$$
\lambda=\frac{Z_{f u} \cdot \varepsilon_{X u}+Z_{f o} \cdot \varepsilon_{X O}+B_{f} \cdot \frac{a}{t} \cdot \varepsilon_{B}}{N \cdot u^{\prime}+0 \cdot\left(-w^{\prime \prime}\right)+Q \cdot \gamma}
$$

zu:

$$
\begin{aligned}
& \lambda=\frac{Z_{f u} \cdot \varepsilon_{x u}+Z_{f 0} \cdot \varepsilon_{x O}+B_{f} \cdot \frac{a}{t} \cdot \frac{1}{2 \cdot \operatorname{tg}^{2} \alpha} \cdot\left(\varepsilon_{x u}+\varepsilon_{x O}\right)}{N \cdot \frac{1}{2} \cdot\left(\varepsilon_{x u}+\varepsilon_{x O}\right)+Q \cdot \frac{1}{\operatorname{tg} \alpha} \cdot\left(\varepsilon_{x u}+\varepsilon_{x O}\right)}= \\
& =\frac{Z_{f u}+\left(Z_{f o}-Z_{f u}\right) \cdot \frac{\varepsilon_{x 0}}{\varepsilon_{x u}+\varepsilon_{x 0}}+B_{f} \cdot \frac{a}{t} \cdot \frac{1}{2 \cdot \operatorname{tg}^{2} \alpha}}{N \cdot \frac{1}{2}+Q \cdot \frac{1}{\operatorname{tg} \alpha}}
\end{aligned}
$$


Man sieht sofort, dass für das vorausgesetzte Armierungsverhältnis $Z_{f o}>Z_{f u}$ der Widerstandsfaktor $\lambda$ unabhängig vom Winkel a für $\varepsilon_{x 0}=0$ ein Minimum annimmt. Damit erhält man folgenden Ausdruck für $\lambda$ :

$$
\lambda=\frac{Z_{f u}+B_{f} \cdot \frac{a}{t} \cdot \frac{1}{2 \cdot \operatorname{tg}^{2} \alpha}}{\frac{1}{2} \cdot N+Q \cdot \frac{1}{\operatorname{tg} \alpha}}
$$

Das Minimum dieser Funktion kann am einfachsten durch Nullsetzen der ersten Ableitung nach der Diagonalenneigung bestimmt werden.

$$
\frac{d \lambda}{d\left(\frac{1}{\operatorname{tg} \alpha}\right)}=\frac{\left(\frac{1}{2} \cdot N+Q \cdot \frac{1}{\operatorname{tg} \alpha}\right) \cdot B_{f} \cdot \frac{a}{t} \cdot \frac{1}{t g \alpha}-\left(Z_{f u}+B_{f} \cdot \frac{a}{t} \cdot \frac{1}{2 \cdot t^{2} \alpha}\right) \cdot Q}{\left(\frac{1}{2} \cdot N+Q \cdot \frac{1}{t g \alpha}\right)^{2}}=0
$$

Da der Nenner dieses Ausdruckes nicht verschwindet, kann der Zähler allein null gesetzt werden. Dies ergibt folgende quadratische Gleichung in $\frac{1}{\operatorname{tg} \alpha}$,

$$
\frac{1}{\operatorname{tg}^{2} \alpha}+\frac{N}{Q} \cdot \frac{1}{\operatorname{tg} \alpha}-\frac{2 \cdot Z_{f u}}{B_{f} \cdot \frac{a}{t}}=0
$$

welche mit Gleichung (68) völlig übereinstimmt. Wie leicht bewiesen werden kann, gelangt man zu einem minimalen widerstandsfaktor $\lambda_{\text {min' wenn die aus }}$ (74) resultierende Diagonalenneigung in (73) eingesetzt wird.

$$
\lambda_{\min }=\frac{N \cdot B_{f} \cdot \frac{a}{t}}{2 \cdot Q^{2}} \cdot\left[-1+\sqrt{\left.1+\frac{B \cdot Z_{f u} \cdot\left(\frac{Q}{N}\right)^{2}}{B_{f} \cdot \frac{a}{t}}\right]}\right.
$$

Beim Vergleich von (70) und (75) stellt man fest, dass die Lösung $\lambda_{\max }$ der Maximumsaufgabe mit der Lösung $\lambda_{\text {min }}$ der Minimumsaufgabe zusammenfällt. Somit 1 iefern beide Programme den exakten Widerstandsfaktor für das angenommene Bruchmodell (siehe Abschnitt 5.1), denn die Lösung der Maximumsaufgabe erfüllt die Gleichgewichts- und Plastizitätsbedingungen und die Lösung der Minimumsaufgabe die kinematischen oder Mechanismusbedingungen.

Folglich ist es gleichgültig, ob die Bestimmung des Querschnittswiderstandes nach der statischen (Abschnitt 5.2) oder nach der kinematischen Methode (Abschnitt 5.3) erfolgt. 
Weil die Lösung der statischen mit jener der kinematischen Aufgabe übereinstimmt (Dualität), muss bei dem nach der statischen Methode erhaltenen Wert des Querschnittswiderstandes ein Querschnitts-Mechanismus eintreten. Das heisst, es besteht die Möglichkeit, die Art der sich ausbildenden Bruchmechanismen aus dem allgemeinen statischen Programm zu erkennen. Dazu wird als erstes das im Abschnitt 5.2 aufgestellte Tableau der statischen Methode (Bild 43) mit den Abkürzungen

$$
\begin{aligned}
& Q_{k}=B_{k} \cdot \frac{a_{k}}{t_{k}} \cdot \frac{1}{\operatorname{tg} \alpha_{k}} \\
& \left|Q_{k}\right|=B_{k} \cdot \frac{a_{k}}{t_{k}} \cdot \frac{1}{\operatorname{tg} \alpha_{k} T} \\
& Q_{f k}=B_{f k} \cdot \frac{a_{k}}{t_{k}} \cdot \frac{1}{\mid t g \alpha_{k}} \mid
\end{aligned}
$$

umgeformt. $Q_{k}$ bedeutet die Schubresultierende der k-ten Querschnittscheibe und $Q_{f k}$ die entsprechende Fliess-Schubkraft, die im wesentlichen eine Funktion der Bügelfliesskraft und der Diagonalenneigung ist. Es sei an dieser Stelle daran erinnert, dass die Bügelkräfte $B_{k}$ nichtnegative Variablen sind, und dass das Vorzeichen der Schubresultierenden $Q_{k}$ allein durch das Vorzeichen des Winkels $\alpha_{k}$ bestimmt wird. Mit Einführung der Beziehungen (76) ergibt sich das in Bild 48 dargestellte Tableau. 

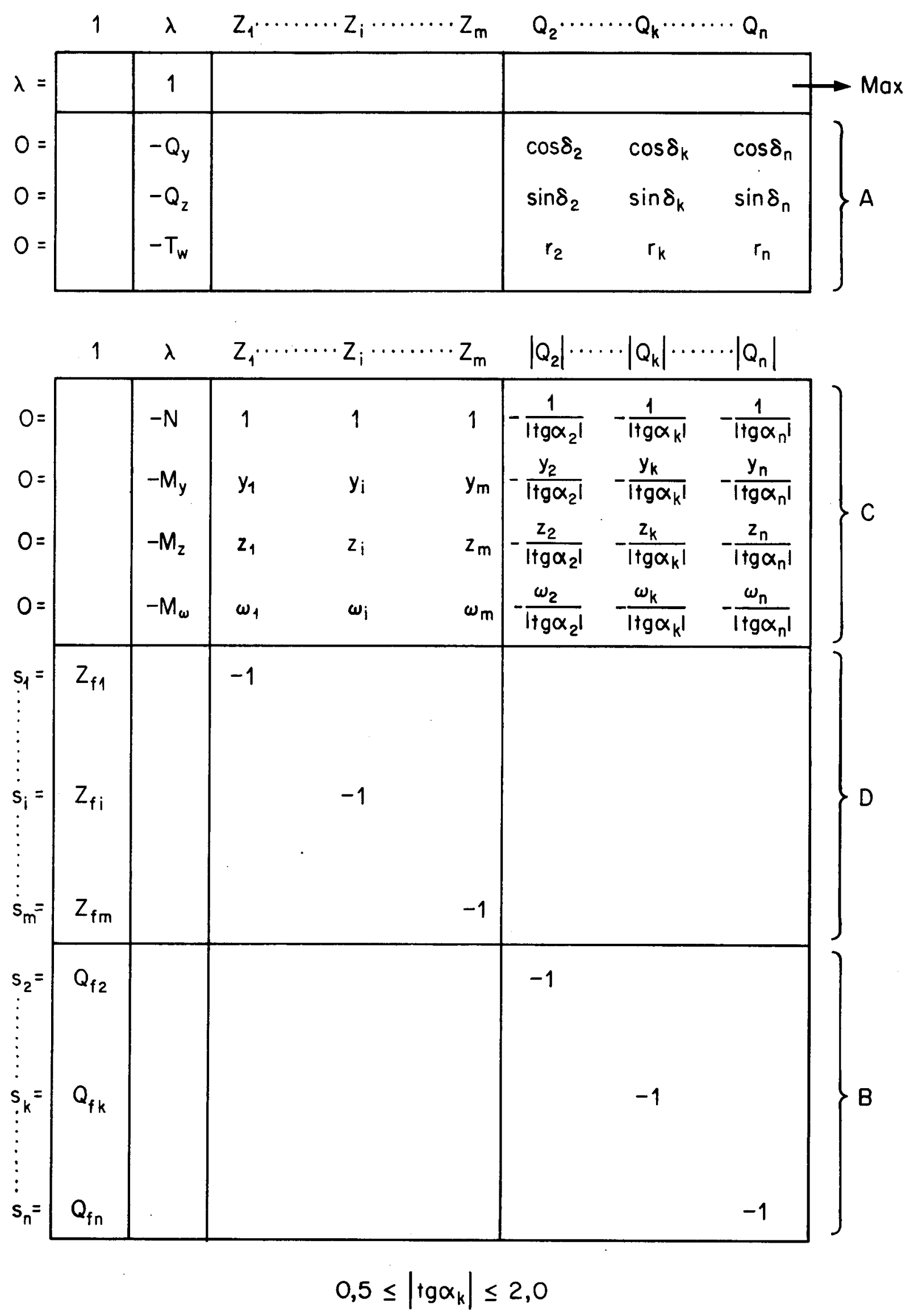
Bevor auf die Bruchmechanismen dünnwandiger offener Stahlbeton- und Spannbetonquerschnitte eingegangen wird, sei kurz an die Beschreibung des Bruchmodelles in Abschnitt 5.1 erinnert. Dort wurde festgestellt, dass bei einem Mechanismus einer Querschnittscheibe im allgemeinen (für $0,5<\left|\operatorname{tg} \alpha_{k}\right|<2,0$ ) Bügel- und Längsarmierung fliessen müssen, und dass beim Erreichen einer der beiden Umlagerungsgrenzen $\left(\left|\operatorname{tg} \alpha_{k}\right|=0,5\right.$ oder 2,0$)$ bereits das Fliessen der Bügel- oder der Längsarmierung zu einem Mechanismus führt. An einer Querschnittscheibe können demnach drei grundsätzlich verschiedene Mechanismen betrachtet werden, je nachdem ob die Schub-, die Längsarmierung oder beide Armierungsanteile ins Fliessen kommen. Für den Querschnitt gilt im Prinzip dasselbe, es kann zwischen drei Arten von Mechanismen unterschieden werden:

1. Längsmechanismus bei Fliessen der Längsarmierung (Gurtstäbe)

2. Schubmechanismus bei Fliessen der Schubarmierung (Bügel)

3. Kombinierter Mechanismus bei Fliessen der Längs- und Schubarmierung

\subsubsection{Längsmechanismus}

Ein solcher Mechanismus kommt zustande, wenn eine starke Schubarmierung lediglich das Fliessen der Gurtstäbe zulässt oder wenn der Querschnitt nur durch die Schnittkräfte $N, M_{y}, M_{z}$ und $M_{w}$ beansprucht wird. Im letzteren Falle sind weder die Querkräfte $Q_{y}$ und $Q_{z}$ noch das Wölbtorsionsmoment $T_{w}$ vorhanden, d.h. auf den Querschnitt wirkt keine Schubbeanspruchung.

Für $Q_{y}=Q_{z}=T_{w}=0$ erfüllt die triviale Lösung $Q_{k}=0(k=2, \ldots, n)$ im Tableau des Bildes 48 sowohl die Gleichgewichtsbedingungen $A$ für den Schubfluss als auch die Plastizitätsbedingungen $B$ für die Bügel. Aus den Gleichgewichtsbedingungen $C$ für die Längskräfte ist im weiteren ersichtlich, dass nichtverschwindende $\left|Q_{k}\right|$ die Gurtstabkräfte $Z_{i}$ erhöhten, d.h. gewisse $Z_{i}$ würden nach den Plastizitätsbedingungen $D$ die obere Grenze $z_{f i}$ früher erreichen.

Die triviale Lösung $Q_{k}=0(k=2, \ldots, n)$ führt somit für den Fall $Q_{y}=Q_{z}=$ $=T_{w}=0$ zum Maximum des Widerstandsfaktors $\lambda$ bzw. zum tatsächlichen Querschnittswiderstand.

Damit reduziert sich das in Bild 48 dargestellte nichtlineare Tableau auf folgendes lineare Unterprogramm. 


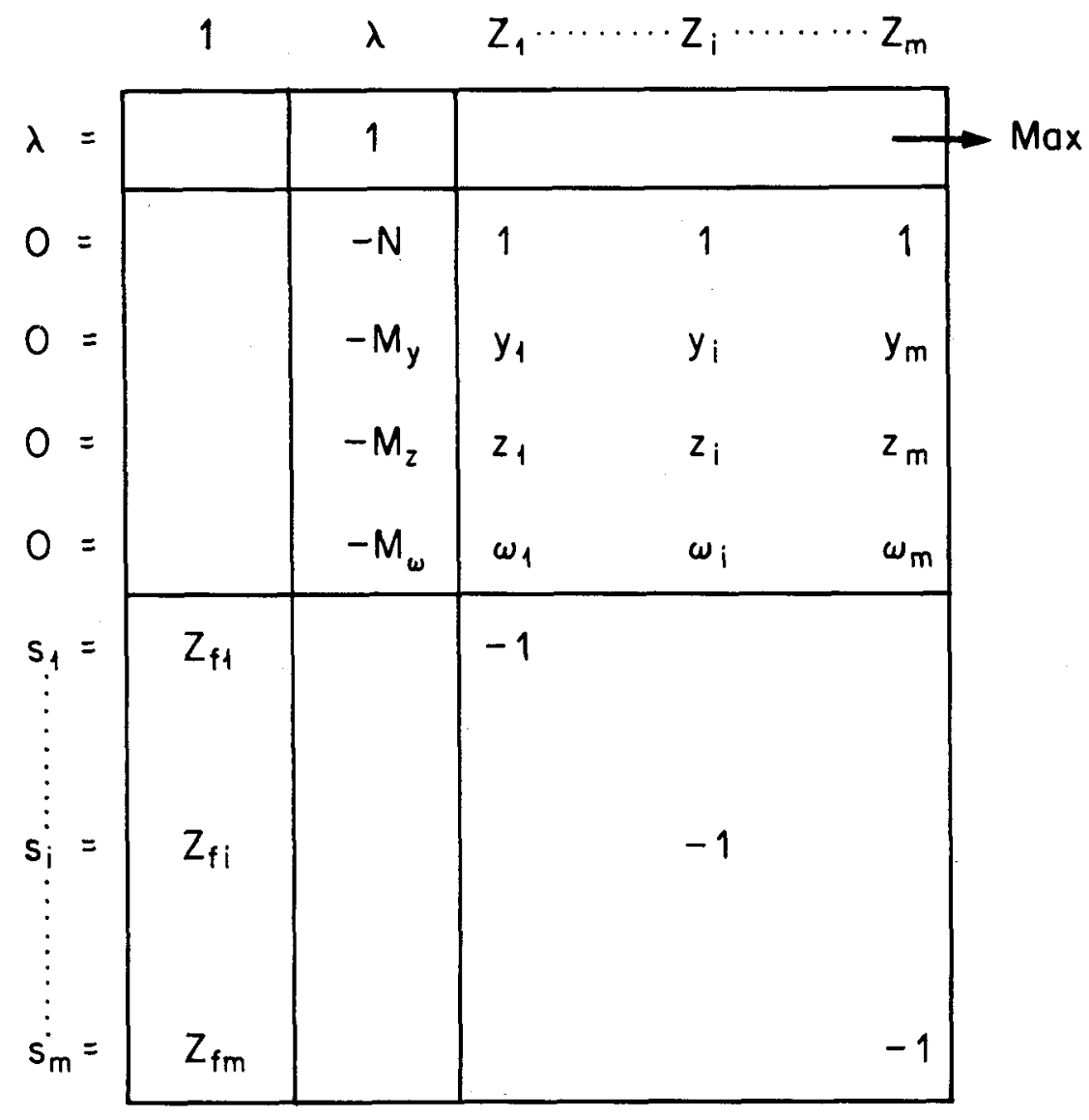

Wie nicht lange bewiesen werden muss, wird das Maximum des Widerstandsfaktors $\lambda$ dann erreicht, wenn von den total $j$ Gurtstäben möglichst viele ins Fliessen kommen.

Die vier Gleichgewichtsbedingungen im Tableau des Bildes 49 weisen $j$ unbekannte Gurtstabkräfte und den unbekannten Widerstandsfaktor $\lambda$ auf, total also (j+1) Unbekannte. Dies führt dazu, dass maximal $(j+1-4)=(j-3)$ Gurtstabkräfte durch ihre Fliesskräfte ersetzt werden dürfen, bis die vier Gleichgewichtsgleichungen mit den ursprünglich $(j+1)$ Unbekannten zu einem regulären Gleichungssystem mit nur noch vier Unbekannten degeneriert sind. Man erhält daraus eindeutige Lösungen für die verbleibenden vier Unbekannten, nämlich den Widerstandsfaktor $\lambda$ sowie die restlichen drei Gurtstabkräfte, die irgendwelche positiven oder negativen Werte annehmen werden. Sie erfüllen, falls die Fliesskräfte bei den richtigen Gurtstäben eingesetzt wurden, die Plastizitätsbedingungen. Im Bruchzustand werden also drei Gurtstabkräfte kleiner oder gleich gross sein wie deren Fliesskräfte, so dass folgende Regel aufgestelit werden kann:

Beim Erreichen des Querschnittswiderstandes müssen alle mit Ausnahme von höchstens drei Gurtstäben fliessen. Betrachtet man die in Abschnitt 5.4 gezeigte Dualität zwischen dem statischen und dem kinematischen Programm, so 
heisst dies, dass bei einem Längsmechanismus normalerweise drei Gurtstäbe starr bleiben.

Zur Illustration dieser Regel mögen die folgenden Beispiele dienen:

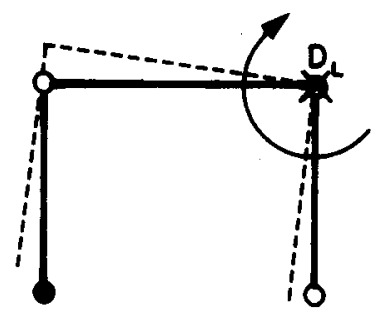

$\mathrm{D}_{\mathrm{L}}$

$X$ : Drehpunkt des Längsmechanismus

0 : starre Gurtstäbe

- : fliessende Gur,stäbe
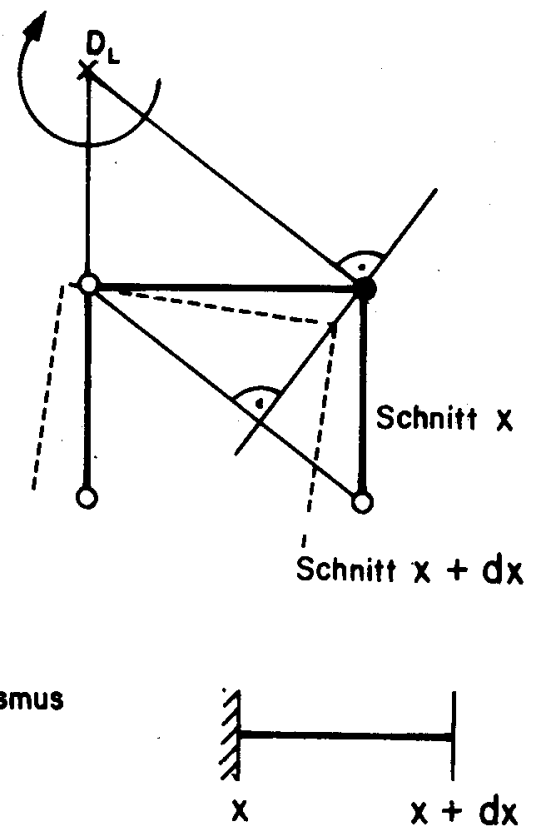

Bild 50

\section{Spezialfälle:}

Eine Ausnahme von der angegebenen Regel kann in jenen Fällen auftreten, wo mehr als zwei Gurtstäbe direkt hintereinander auf einer Geraden liegen, zum Beispiel:

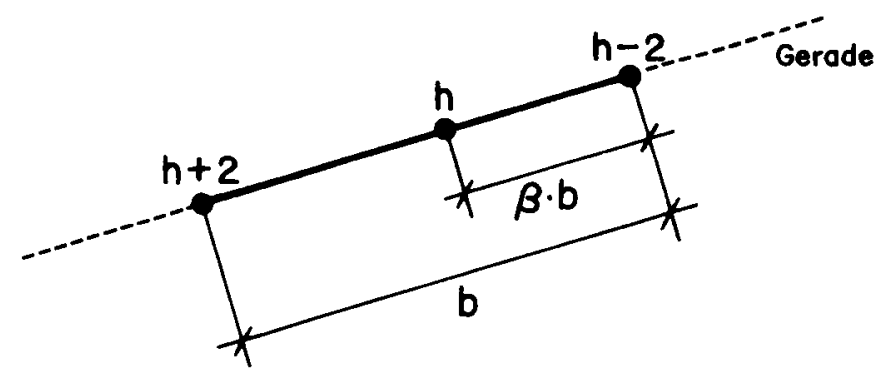

- : Gurtstäbe

- : Querschnittscheiben 
Die Frage, was in diesem Fall passiert, kann am einfachsten mit Hilfe des kinematischen Programmes beantwortet werden, das zum Iinearen Tableau des Bildes 49 dual ist.

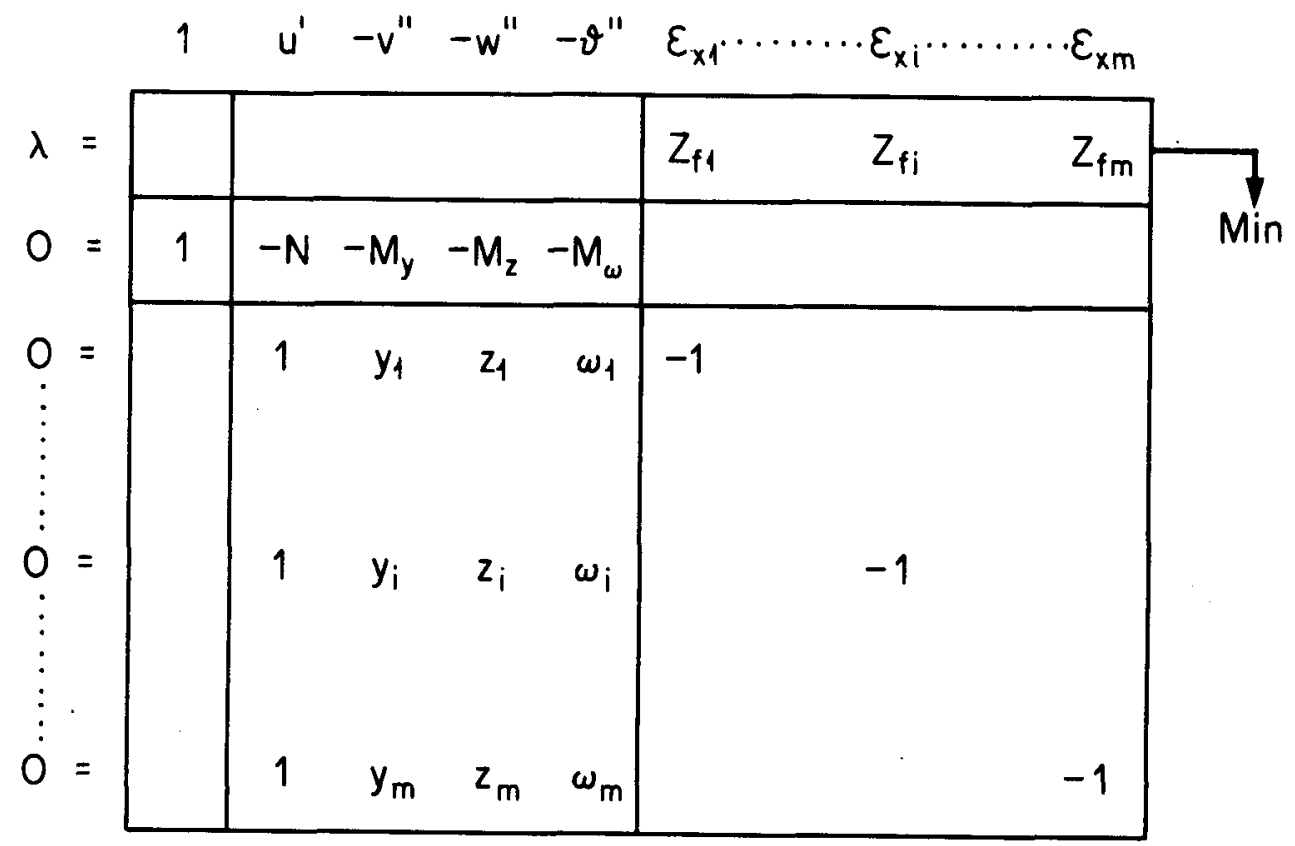

Liegt ein Gurtstab h auf der Verbindungsgeraden zwischen zwei Gurtstäben $h-2$ und $h+2$, so sind dessen Koordinaten $y_{h}, z_{h}$ und $\omega_{h}$ Linearkombinationen der Koordinaten der beiden Gurtstäbe $h-2$ und $h+2$. Das heisst mit Blick auf das oben angegebene Tableau, dass auch die Dehnung $\varepsilon_{x h}$ eine Linearkombination der Dehnungen $\varepsilon_{x h-2}$ und $\varepsilon_{x h+2}$ sein muss.

Es gilt

$$
\left.\begin{array}{rl}
\text { für: } \varepsilon_{x h-2}>0 \\
\varepsilon_{x h+2}>0
\end{array}\right\} \rightarrow \varepsilon_{x h}>0
$$

für: $\varepsilon_{x h-2}>0$

$\varepsilon_{\times h+2}=0$

bzw.

$$
\rightarrow \varepsilon_{x h}>0
$$$$
\varepsilon_{x h-2}=0
$$$$
\varepsilon_{\times h+2}>0
$$

für: $\varepsilon_{x h-2}=0$

$$
\varepsilon_{x h+2}=0
$$


Wie aus Bild 53 hervorgeht, kann für den Fall, dass mehr als zwei starre Gurtstäbe direkt hintereinander auf einer Geraden liegen, ein Mechanismus auftreten, bei dem im ganzen mehr als drei Gurtstäbe starr bleiben.
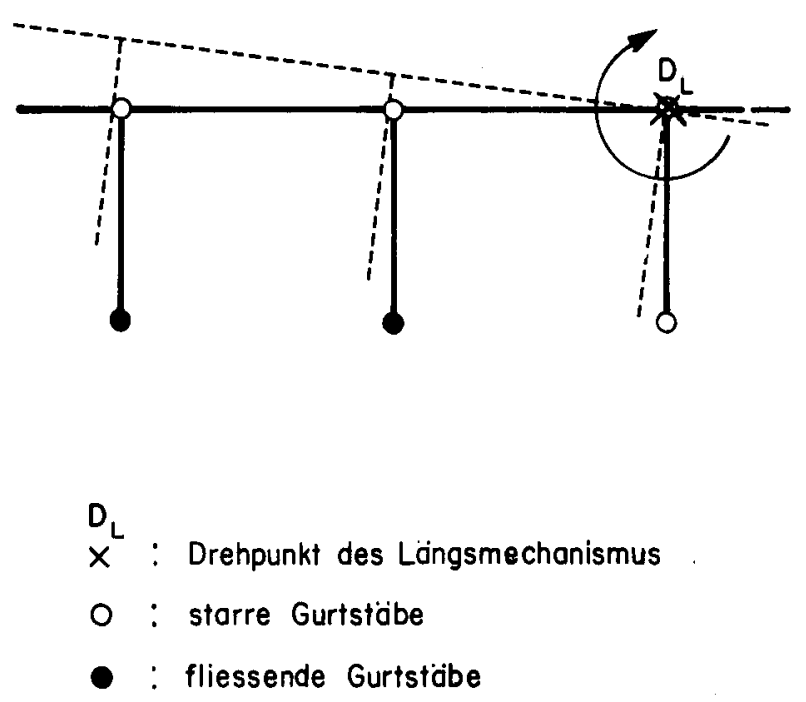

Bild 53

Dann können die vier zur Verfügung stehenden Gleichgewichtsbedingungen nicht mehr zu einem regulären Gleichungssystem reduziert werden, da in vier Gleichungen mehr als vier Unbekannte (vier Gurtstabkräfte sowie der Widerstandsfaktor $\lambda$ ) vorkommen. Der Querschnittswiderstand lässt sich zwar bestimmen, nicht aber die Aufteilung der Kräfte auf die starr bleibenden Gurtstäbe des statisch unbestimmten Restquerschnittes. Dasselbe gilt bekanntlich auch für lokale Tragwerkmechanismen, bei denen die Traglast eindeutig gegeben, die Schnittkraftverteilung am statisch unbestimmten Restsystem für starr-plastisches Materialverhalten jedoch nicht definiert ist.

Interessieren neben dem Querschnittswiderstand auch die einzelnen Gurtstabkräfte (z.B. für die Abschätzung der Sprödbruchgefahr), so kann man sich auf sehr einfache weise behelfen, indem anstelle eines starr-plastischen ein elastisch-plastisches Materialverhalten vorausgesetzt wird. Nach starrplastischer Theorie sind die Dehnungen für Gurtstäbe, welche ihre Fliessgrenze nicht erreichen, voraussetzungsgemäss gleich null. In Wirklichkeit nehmen sie jedoch von null verschiedene Werte an und sind aufgrund der kinematischen Bedingungen linear zwischen den Gurtstäben $h-2$ und $h+2$ verteilt. 


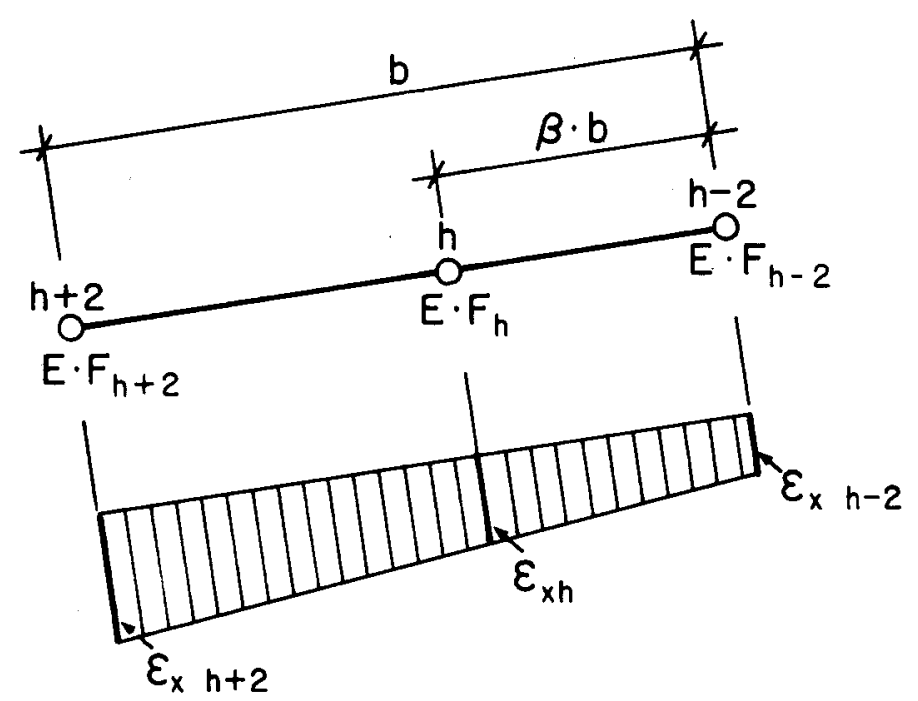

\section{O: starre Gurtstäbe gemäss starr-plastischer Theorie \\ - : Querschnittscheiben \\ E: Elastizitätsmodul \\ $F_{h}:$ Querschnittsfläche des Gurtstabes $h$}

(bei Druckbeanspruchung ideelle Querschnittsfläche inklusive Beton)

Die Gurtstäbe $h-2$ bis $h+2$ befinden sich bei Annahme eines elastisch-plastischen Stoffgesetzes im elastischen Zustand.

$$
\begin{aligned}
& \varepsilon_{x h}=(1-\beta) \cdot \varepsilon_{x h-2}+\beta \cdot \varepsilon_{x h+2} \\
& z_{h-2}=E \cdot F_{h-2} \cdot \varepsilon_{\times h-2} \\
& z_{h}=E \cdot F_{h} \cdot \varepsilon_{x h} \\
& z_{h+2}=E \cdot F_{h+2} \cdot \varepsilon_{\times h+2}
\end{aligned}
$$

Folglich lässt sich die Gurtstabkraft $z_{h}$ in Funktion von $z_{h-2}$ und $z_{h+2}$ ausdrücken:

$$
Z_{h}=E \cdot F_{h} \cdot\left[(1-\beta) \cdot \varepsilon_{x h-2}+\beta \cdot \varepsilon_{x h+2}\right]
$$

oder

$$
Z_{h}=\underbrace{(1-\beta) \cdot \frac{F_{h}}{F_{h-2}}}_{\text {Faktor }} \cdot Z_{h-2}+\underbrace{\beta \cdot \frac{F_{h}}{F_{h+2}}}_{\text {Faktor }} \cdot Z_{h+2}
$$


Dank dieser zusätzlichen Gleichung erhält man schliesslich auch für den geschilderten Spezialfall aus den Gleichgewichtsbedingungen ein reguläres Gleichungssystem mit einem eindeutig bestimmten Lösungsvektor für den Widerstandsfaktor $\lambda$ und die bis anhin unbekannten Gurtstabkräfte.

\subsubsection{Schubmechanismus}

Wird bei einem auf Schub ( $Q_{y}, Q_{z}, T_{w}$ ) beanspruchten Querschnitt, auf den auch eine Längsbeanspruchung ( $N, M_{y}, M_{z}, M_{\omega}$ ) wirken kann, jeglicher Längsmechanismus durch eine starke Längsarmierung, d.h. durch starke Gurtstäbe verhindert, so wird beim Erreichen des Querschnittswiderstandes ein sogenannter Schubmechanismus eintreten.

Diese Annahme vorausgesetzt, können die Gleichgewichtsbedingungen $C$ und die Plastizitätsbedingungen 0 für die Längskräfte im Tableau des Bildes 48 in jedem Falle erfüllt werden. Sie haben demnach keinen Einfluss auf den Querschnittswiderstand und können weggelassen werden.

Zur Bestimmung des Widerstandsfaktors $\lambda$ genügt in diesem Falle folgendes einfache Programm.
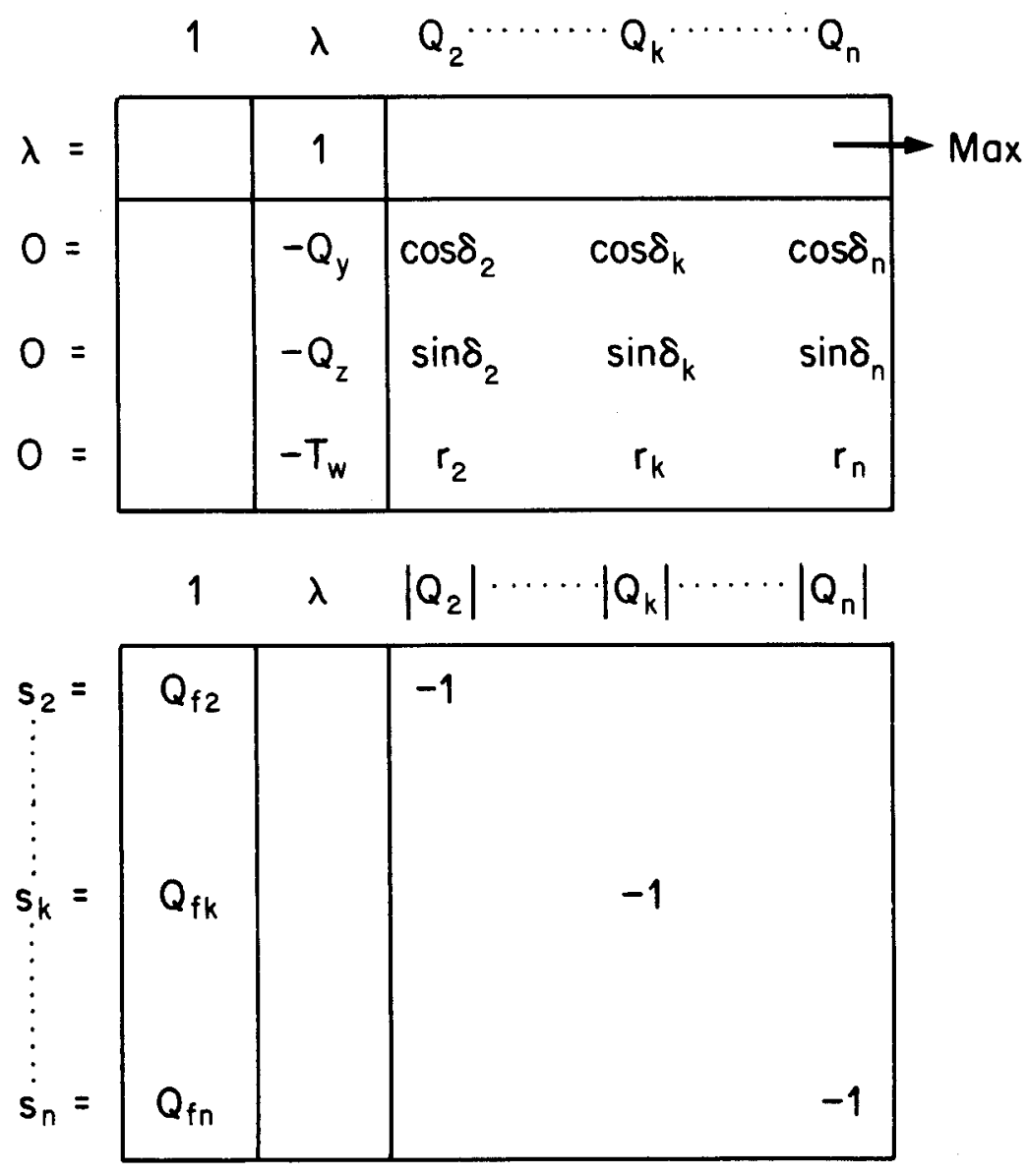
Die Fliess-Schubkraft $Q_{f k}$ der k-ten Querschnittscheibe ist im wesentlichen eine Funktion der Bügelfliesskraft $B_{f k}$ und der Diagonalenneigung $\alpha_{k}$.

$$
Q_{f k}=B_{f k} \cdot \frac{a_{k}}{t_{k}} \cdot \frac{1}{\left|t g \alpha_{k}\right|}
$$

Bleibt, wie hier vorausgesetzt, die Längsarmierung starr, so kann $Q_{f k} m i t$ dem minimalen Winkel $\alpha_{k}$ bestimmt werden, da die Schubarmierung bei kleinster Diagonalenneigung $\alpha_{k}$ am wirksamsten ist und demzufolge der Widerstandsfaktor $\lambda$ maximal wird. Dies führt nach (53) dazu, dass hier die Variablen $\frac{1}{\sqrt{\operatorname{tg} \alpha_{k}}}$ durch den konstanten wert 2 ersetzt werden dürfen.

$$
Q_{f k}=2 \cdot B_{f k} \cdot \frac{a_{k}}{t_{k}}
$$

Das nichtlineare Programm des Bildes 55 wird damit linear, und für den Schubmechanismus gilt, wie ohne Herleitung einzusehen ist, folgende Regel:

Beim Erreichen des Querschnittswiderstandes fliessen die Bügel in allen ausser höchstens zwei Querschnittscheiben. Das heisst, dass bei einem Schubmechanismus im Normalfall die Bügel zweier Querschnittscheiben starr bleiben.

Diese Gesetzmässigkeit soll an folgendem Beispiel demonstriert werden:

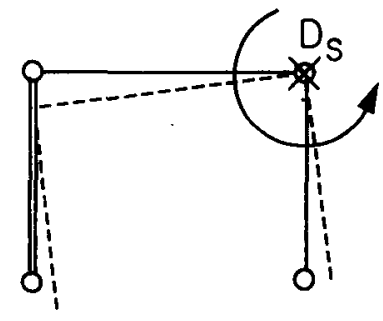

positive

Diagonalenneigung

$D_{S}$

$X$ : Drehpunkt des Schubmechanismus

- : starre Bügel

= fliessende Bügel

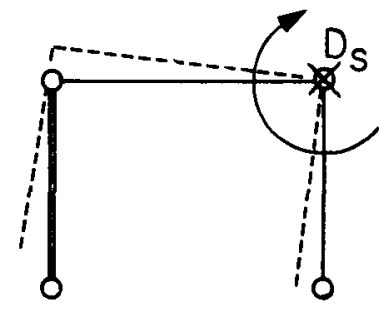

negative

Diagonnalenneigung 


\section{Spezialfälle:}

Eine Ausnahme von der angegebenen Regel kann wie beim Längsmechanismus in jenen Fällen auftreten, wo mehr als zwei Gurtstäbe bzw. mehr als eine Querschnittscheibe direkt hintereinander auf einer Geraden liegen.
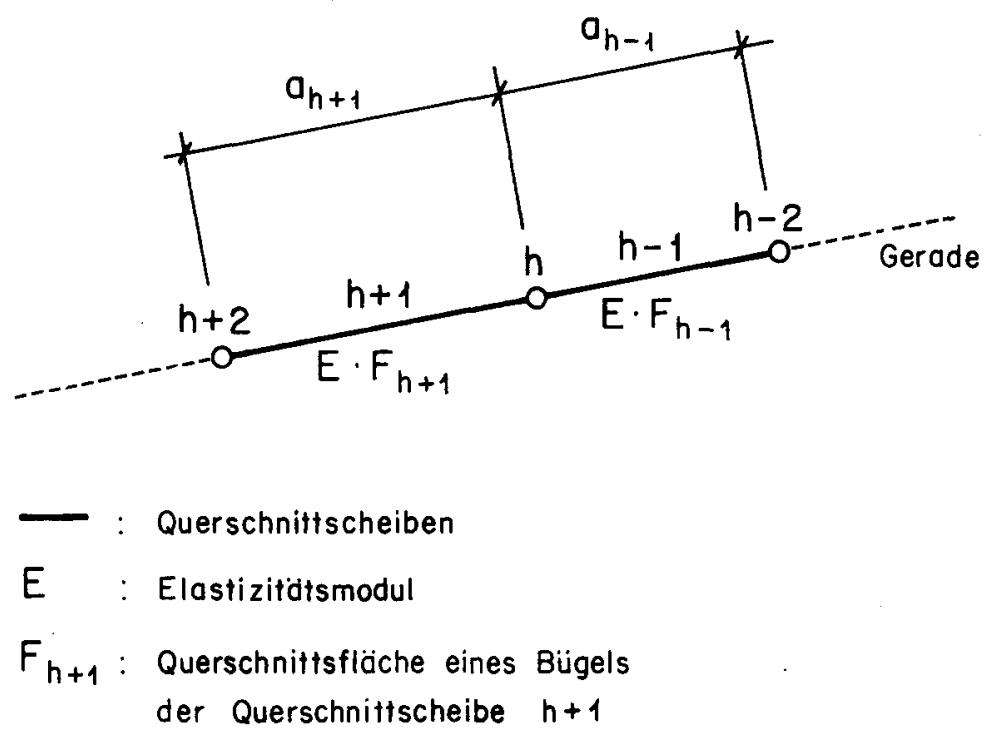

Bild 57

Die kinematischen Bedingungen verlangen, dass die Schiebungen der beiden Querschnittscheiben $h-1$ und $h+1$ gleich gross sind.

$$
\gamma_{h+1}=\gamma_{h-1}
$$

Unter der hier gültigen Voraussetzung starrer Gurtstäbe ergibt sich bei minimaler Diagonalenneigung aus (52b) ein direkter Zusammenhang zwischen Bügeldehnung und Schiebung.

$$
\begin{aligned}
\text { für } \gamma_{k}>0: & \gamma_{k}=\varepsilon_{B k} \cdot \operatorname{tg} \alpha_{k}=0,5 \cdot \varepsilon_{B k} \\
\gamma_{k}<0: & \gamma_{k}=\varepsilon_{B k} \cdot \operatorname{tg} \alpha_{k}=-0,5 \cdot \varepsilon_{B k}
\end{aligned}
$$

Demzufolge entsprechen sich auch die Bügeldehnungen der Querschnittscheiben $h-1$ und $h+1$.

$$
\varepsilon_{B h+1}=\varepsilon_{B h-1}
$$

Für den Spezialfall starrer Bügel in den Querschnittscheiben $h-1$ und $h+1$ kann, wie aus Bild 58 hervorgeht, ein Mechanismus auftreten, bei dem die 
Bügel von mehr als zwei Querschnittscheiben starr und die betreffenden'Bügelkräfte nach starr-plastischer Theorie unbestimmt bleiben.

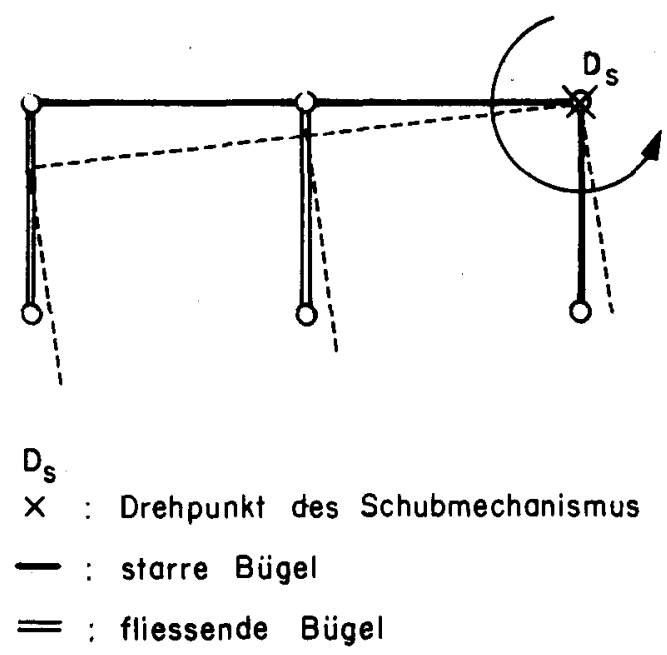

Bild 58

Bei gleichem Vorgehen wie bei den Gurtstabkräften, wo $Z_{h}$ mit Hilfe eines elastisch-plastischen Materialverhaltens in Funktion von $Z_{h-2}$ und $Z_{h+2}$ ausgedrückt werden konnte, ist es hier möglich, die Schubkraft $Q_{h+1}$ als Funktion der Schubkraft $Q_{h-1}$ darzustellen.

$$
Q_{h+1}=\frac{F_{h+1} \cdot \frac{a_{h+1}}{t_{h+1}}}{F_{h-1} \cdot \frac{a_{h-1}}{t_{h-1}}} \cdot Q_{h-1}
$$

Damit kann aus den drei Gleichgewichtsbedingungen für den Schubfluss bzw. die Schubresultierenden $Q_{k}$ im Tableau des Bildes 55 auch für den in Bild 58 dargestellten Mechanismus ein reguläres Gleichungssystem erhalten werden, was die eindeutige Bestimmung der Bügelkräfte im Bruchzustand ermöglicht.

\section{5 .3 Kombinierter Mechanismus}

Ein solcher Mechanismus tritt ein, wenn das Verhältnis der Längs- und der Bügelarmierung so gewählt ist, dass beide Armierungsanteile ins Fliessen kommen. Für diesen Fall gilt die in (52a) angegebene Koppelung der Bügelund Längsdehnungen, womit der kombinierte Mechanismus direkt aus dem zu Beginn dieses Abschnittes besprochenen Längsmechanismus abgeleitet werden kann. Es gilt im allgemeinen folgende Gesetzmässigkeit. 
Bei einem kambinierten Mechanismus kommen alle ausser höchstens drei Gurtstäben ins Fliessen. Zudem fliessen die Bügel in jenen Querschnittscheiben, die sich an einen fliessenden Gurtstab anschliessen.

Ausnahmen, wo die Bügel auch in einer neben einem fliessenden Gurtstab liegenden Scheibe starr bleiben, sind ziemlich häufig. Wird nämlich in einzelnen Querschnittscheiben eine der beiden Umlagerungsgrenzen $\left|\operatorname{tg} \alpha_{k}\right|=0,5$ bzw. 2,0 erreicht, so gilt die Koppelung der Bügel- und Längsdehnungen nicht mehr über den gesamten Querschnitt. Dies ist immer der Fall, wenn die Querschnittscheiben sehr unterschiedliche Schubbeanspruchungen aufweisen, wofür jener Grenzfall typisch ist, bei dem die Schubresultierende einer einzelnen Querschnittscheibe verschwindet.

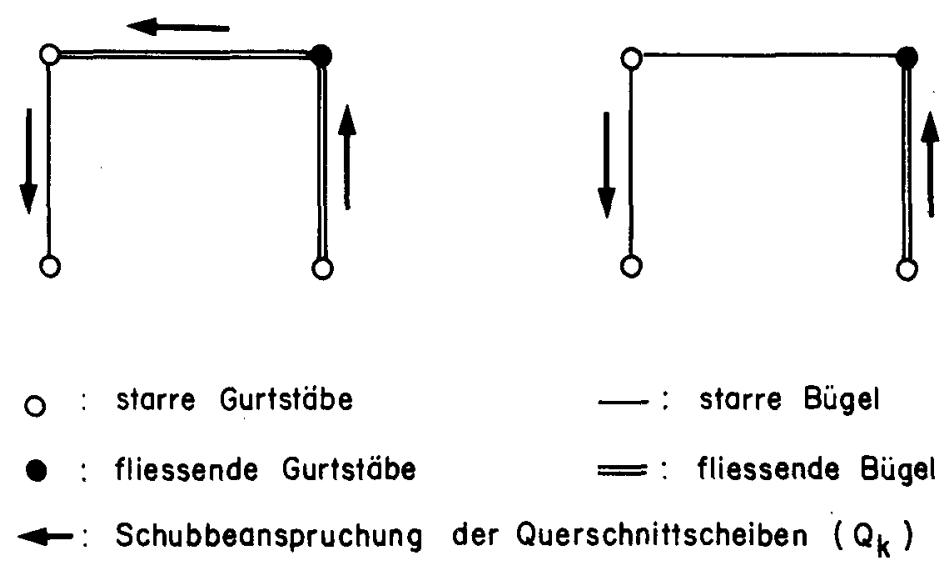

Bild 59

Mit dem Hinweis auf die zahlreichen Spezialfälle, welche sich aufgrund der beschränkten Diagonalenneigung (siehe oben) ergeben, kann die Besprechung der kombinierten Mechanismen als abgeschlossen betrachtet werden.

\section{Zusammenstellung der Ergebnisse:}

Nach der Beschreibung der verschiedenen Querschnitts-Mechanismen scheint eine Zusammenstellung der wichtigsten Ergebnisse zwecks besserer Uebersicht angebracht.

1. Beim Erreichen des Querschnittswiderstandes ist entweder ein Längs-, ein Schub- oder ein kombinierter Mechanismus möglich.

2. Bei einem kombinierten Mechanismus kommen die Längs- und die Schubarmierung ins Fliessen. Ein Längsmechanismus (Fliessen der Längsarmierung) kommt zustande, wenn entweder die Schubbeanspruchung fehlt oder eine starke Schubarmierung lediglich ein Fliessen der Gurtstäbe zulässt ( $\operatorname{tg} \alpha=2,0)$. Wird hingegen durch 
eine starke Längsarmierung ein Fliessen der Gurtstäbe verhindert, so tritt ein Schubmechanismus (Fliessen der Schubarmierung) ein $(\operatorname{tg} \alpha=0,5)$.

3. Bei einem kombinierten Mechanismus bleiben im Normalfall drei Gurtstäbe starr. Die Bügel bleiben meistens nur in jenen Querschnittscheiben starr, die zwischen zwei starren Gurtstäben liegen.

4. Bei einem Längsmechanismus bleiben neben der Schubarmierung im allgemeinen drei Gurtstäbe starr.

5. Bei einem Schubmechanismus bleiben neben der Längsarmierung normalerweise die Bügel von zwei Querschnittscheiben starr.

Für Spezialfälle und Ausnahmen von diesen Regeln sei auf den vorhergehenden Text verwiesen.

\subsection{Praktische Traglastbestimmung}

Es wäre ohne weiteres möglich, eines der in den Abschnitten 5.2 und 5.3 erhaltenen nichtlinearen Programme auf irgendeine Art zu linearisieren (Näherung) und dann numerisch zu lösen, doch ist dieser Weg für die gestellte Aufgabe viel zu aufwendig. Im folgenden wird eine einfache Näherungsmethode entwickelt, welche auf der Kenntnis der Querschnitts-Mechanismen basiert.

\subsubsection{Prinzipielles Vorgehen}

Die in Abschnitt 5.6.2 entwickelte Berechnungsmethade baut auf einem Vorgehen auf, welches in der Plastizitätstheorie zur Lösung linearer probleme geläufig ist. Dieses Vorgehen soll zur Einführung am Beispiel reiner Längsbeanspruchung kurz erläutert werden.

Für den Spezialfall reiner Längsbeanspruchung $\left(Q_{y}=Q_{z}=T_{w}=0\right)$ tritt ein sogenannter Längsmechanismus ein, wie er im vorhergehenden Abschnitt beschrieben ist. Man schätzt nun den wahrscheinlichsten Längsmechanismus und bestimmt den Querschnittswiderstand bzw. den Widerstandsfaktor unter der bewiesenen Voraussetzung, dass mit Annahme von drei Gurtstäben alle ihre 
Fliessgrenze erreichen. Die Lösung der Gleichgewichtsbedingungen ergibt dann neben dem Widerstandsfaktor die drei unbekannten Gurtstabkräfte. Sind diese kleiner oder höchstens gleich gross wie ihre Fliesskräfte, so sind die Plastizitätsbedingungen erfüllt und der exakte Wert des Querschnittswiderstandes ist gefunden. Wird jedoch eine dieser Plastizitätsbedingungen verletzt, so hat man eine obere Schranke erhalten. Die Berechnung ist in dem Fall mit einem neu angenommenen Mechanismus zu wiederholen, bis alle Plastizitätsbedingungen erfüllt sind, d.h. bis der exakte Querschnittswiderstand gefunden ist.

Der beschriebene analytische Lösungsweg ist im allgemeinen sehr rasch, da bei den häufig vorkommenden Belastungsfällen und Querschnitten der Bruchmechanismus meist auf Anhieb richtig geschätzt werden kann.

\subsubsection{Berechnungsmethode}

Im allgemeinen nichtlinearen Fall kann der Querschnittswiderstand nach einer Näherungsmethode bestimmt werden, welche auf dem vorher geschilderten Vorgehen aufbaut. Sie besitzt den Vorteil, einfach und übersichtlich zu sein und trotzdem gute Resultate zu liefern.

Als Basis für die Berechnung dienen die im vorangehenden Abschnitt aufgestellten Regeln für den Längs- und den Schubmechanismus. Es wird absichtlich nicht von einem kombinierten Mechanismus ausgegangen, da für eine auf diese Weise bestimmte Näherungslösung immer mit dem vollständigen Programm, d.h. mit sieben Gleichgewichtsbedingungen gleichzeitig gearbeitet werden müsste. Bei der im folgenden beschriebenen Methode werden die beiden Unterprogramme für die Längs- und die Schubbeanspruchung einzeln gelöst und erst am Schluss miteinander gekoppelt. Die gesamte Berechnung kann in drei Schritte unterteilt werden.

\section{Erster Schritt:}

In einem ersten Schritt wird das aus dem Tableau des Bildes 48 herausgelöste und in Bild 60 dargestellte Unterprogramm für die Schubbeanspruchung behandelt. 


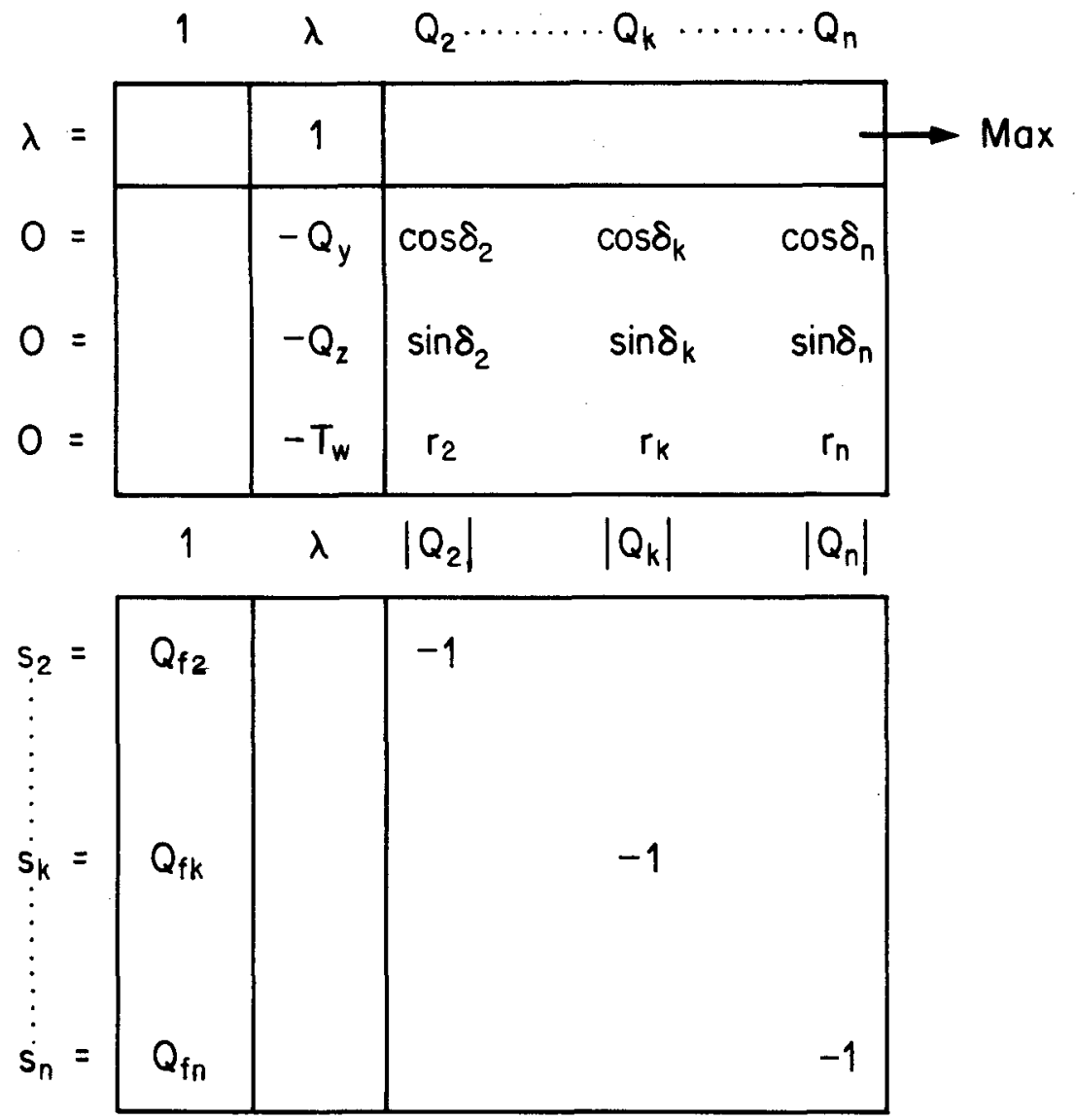

Bild 60

In diesem Programm kommen neben dem Widerstandsfaktor $\lambda$ und den Schubresultierenden $Q_{k}$ der einzelnen Querschnittscheiben auch noch die in den FliessSchubkräften $Q_{f k}$ enthaltenen Diagonalenneigungen $\alpha_{k}$ als Unbekannte vor, welche eine einfache Lösung des Problems verunmöglichen. Aus diesem Grunde werden die in Wirklichkeit von Scheibe zu Scheibe ändernden winkel $\alpha_{k}$ durch eine über den ganzen Querschnitt dem Betrag nach gleich angenommene Diagonalenneigung $\alpha$ ersetzt. Man beschränkt dadurch die volle Umlagerungsmöglichkeit und wird immer eine untere Schranke des Querschnittswiderstandes erhalten, was angesichts der Tatsache, dass sich ein sehr handiches Berechnungsverfahren ergibt, jedoch nicht ins Gewicht fällt. 
Mit Einführung einer mittleren Diagonalenneigung $\alpha$ transformieren sich die in (76) angegebenen Schubkräfte zu:

$$
\begin{aligned}
& Q_{k}=B_{k} \cdot \frac{a_{k}}{t_{k}} \cdot \frac{1}{\operatorname{tg} \alpha}=\bar{Q}_{k} \cdot \frac{1}{|t g \alpha|} \\
& \left|Q_{k}\right|=B_{k} \cdot \frac{a_{k}}{t_{k}} \cdot \frac{1}{|t g \alpha|}=\left|\bar{Q}_{k}\right| \cdot \frac{1}{|t g \alpha|} \\
& Q_{f k}=B_{f k} \cdot \frac{a_{k}}{t_{k}} \cdot \frac{1}{|t g \alpha|}=\bar{Q}_{f k} \cdot \frac{1}{|t g \alpha|}
\end{aligned}
$$

Weil nach (77) alle Fliess-Schubkräfte Funktionen desselben Winkels $\alpha$ sind, kann die Lösung wie für einen reinen Schubmechanismus erfolgen. Werden die Schubresultierenden in allen ausser zwei Querschnittscheiben $A$ und $B$ durch die betreffenden Fliess-Schubkräfte ersetzt, so erhält man aus den im Tableau des Bildes 60 enthaltenen Gleichgewichtsbedingungen ein reguläres Gleichungssystem.

$$
\left[\begin{array}{c:cc}
-Q_{y} & \cos _{A} & \cos _{B} \\
-Q_{Z} & \sin \delta_{A} & \sin _{B} \\
-T_{W} & r_{A} & r_{B}
\end{array}\right] \cdot\left\{\begin{array}{c}
\lambda \\
Q_{A} \\
Q_{B}
\end{array}\right\}=\left\{\begin{array}{c}
-q_{1} \\
-q_{2} \\
-q_{3}
\end{array}\right\} \cdot \frac{1}{\mid t g \alpha}
$$

[Q]

Dabei ergibt sich die rechte Seite dieses Gleichungssystems aus den FliessSchubkräften der massgebenden Querschnittscheiben (k=fk), d.h. aller Querschnittscheiben ausser $A$ und $B$. Die von ihnen aufgenommenen Schnittkraftanteile an $Q_{y}, Q_{z}$ und $T_{w}$ kammen als Belastungsglieder vor, wobei die Koeffizienten $q_{1}$ bis $q_{3}$ (vgl. (77)) Abkürzungen der folgenden Summen sind.

$$
\begin{aligned}
& q_{1}=\sum_{k=f k} \bar{Q}_{k} \cdot \cos \delta_{k} \\
& q_{2}=\sum_{k=f k} \bar{Q}_{k} \cdot \sin \delta_{k} \\
& q_{3}=\sum_{k=f k} \bar{Q}_{k} \cdot r_{k}
\end{aligned}
$$


Die Lösungen des Gleichungssystems

$$
\left\{\begin{array}{l}
\lambda \\
Q_{A} \\
Q_{B}
\end{array}\right\}=[Q]^{-1} \cdot\left\{\begin{array}{l}
-a_{1} \\
-a_{2} \\
-a_{3}
\end{array} \mid \cdot \frac{1}{\operatorname{tg} \alpha}\right.
$$

sind Funktionen der mittleren Diagonalenneigung $\alpha$.

$$
\lambda=\bar{\lambda} \cdot \frac{1}{|\operatorname{tg} \alpha|}
$$

sowie:

$$
\begin{aligned}
& Q_{A}=\bar{Q}_{A} \cdot \frac{1}{|\operatorname{tg} \alpha|} \\
& Q_{B}=\bar{Q}_{B} \cdot \frac{1}{|\operatorname{tg} \alpha|}
\end{aligned}
$$

Wie aus (77) entnommen werden kann, sind die Plastizitätsbedingungen für die Schub-, resp. Bügelkräfte dann erfüllt, wenn die Ungleichungen

$$
\begin{aligned}
& \left|\bar{Q}_{A}\right| \leq \bar{Q}_{f A} \\
& \left|\bar{Q}_{B}\right| \leq \bar{Q}_{f B}
\end{aligned}
$$

eingehalten werden.

\section{Zweiter Schritt:}

In diesem zweiten Schritt wird der Spannungszustand in Stablängsrichtung untersucht. Dabei sind selbstverständlich auch die aus der Schubbeanspruchung resultierenden Längskräfte zu berücksichtigen.

$$
z_{k}=\left|Q_{k}\right| \cdot \frac{1}{T \operatorname{tg} \alpha \mid}=\left|\bar{Q}_{k}\right| \cdot \frac{1}{\operatorname{tg}^{2} \alpha}
$$


Mit den Abkürzungen

$$
\begin{aligned}
& b_{1 \alpha}=\sum_{k=2}^{n}\left|\bar{Q}_{k}\right| \\
& b_{2 \alpha}=\sum_{k=2}^{n}\left|\bar{Q}_{k}\right| \cdot y_{k} \\
& b_{3 \alpha}=\sum_{k=2}^{n}\left|\bar{Q}_{k}\right| \cdot z_{k} \\
& b_{4 \alpha}=\sum_{k=2}^{n}\left|\bar{Q}_{k}\right| \cdot \omega_{k}
\end{aligned}
$$

erhält man aus den Gleichgewichts- und Plastizitätsbedingungen für die Längskräfte (siehe Tableau des Bildes 48) folgendes Programm für den zu maximierenden Widerstandsfaktor $\lambda$.

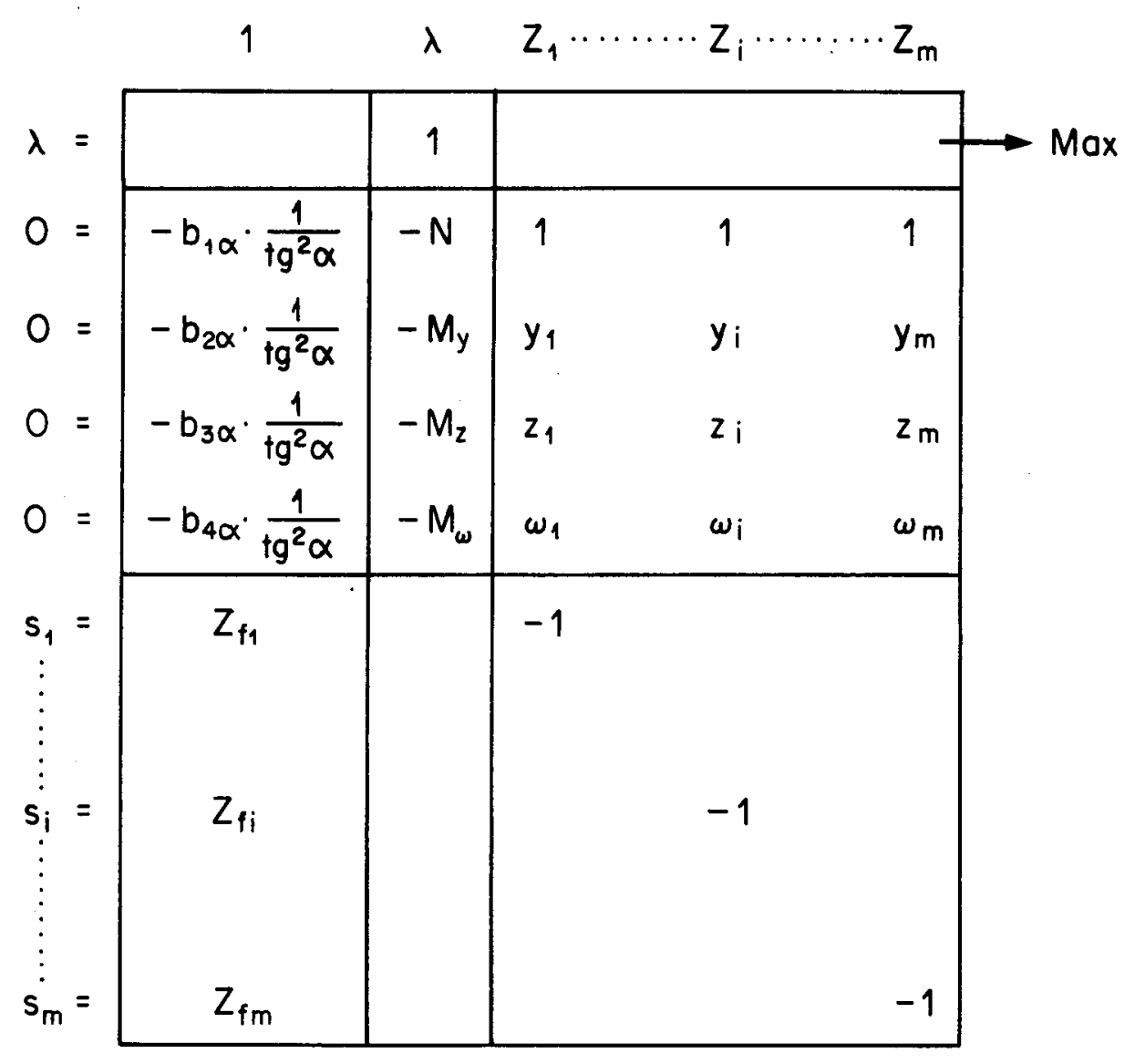

Bild 61

Die Läsung dieses Programmes kann wie für einen reinen Längsmechanismus erfolgen, indem alle ausser drei Gurtstäben $D$, E und F ihre Fliesskräfte erreichen. In diesem Fall ergibt sich aus den vier Gleichgewichtsgleichungen im Tableau des Bildes 61 ein Gleichungssystem von folgender Form. 


$$
\left[\begin{array}{c:ccc}
-N & 1 & 1 & 1 \\
-M_{y} & y_{D} & y_{E} & y_{F} \\
-M_{z} & z_{D} & z_{E} & z_{F} \\
-M_{w} & w_{D} & \omega_{E} & \omega_{F}
\end{array}\right] \cdot\left\{\begin{array}{c}
\lambda \\
z_{D} \\
z_{E} \\
z_{F}
\end{array}\right\}=\left\{\begin{array}{c}
-b_{1} \\
-b_{2} \\
-b_{3} \\
-b_{4}
\end{array}\right\}+\left\{\begin{array}{c}
b_{1 \alpha} \\
b_{2 \alpha} \\
b_{3 \alpha} \\
b_{4 \alpha}
\end{array}\right\} \cdot \frac{1}{t^{2} \alpha}
$$

$[\mathrm{M}]$

Als Belastungsglieder kommen neben $b_{1 \alpha}$ bis $b_{4 \alpha}$, welche durch die Schubbeanspruchung bedingt sind, die Ausdrücke $b_{1}$ bis $b_{4}$ vor. Sie resultieren aus den fliessenden Gurtstäben ( $i=f i$ ) und drücken die von ihnen aufgenommenen Schnittkraftanteile an $N, M_{y}, M_{z}$ und $M_{w}$ aus.

$$
\begin{aligned}
& b_{1}=\sum_{i=f i} z_{i} \\
& b_{2}=\sum_{i=f i} z_{i} \cdot y_{i} \\
& b_{3}=\sum_{i=f i} z_{i} \cdot z_{i} \\
& b_{4}=\sum_{i=f i} z_{i} \cdot w_{i}
\end{aligned}
$$

Wie im ersten Schritt sind auch hier die Lösungen des Gleichungssystems

$$
\left\{\begin{array}{l}
\lambda \\
z_{D} \\
Z_{E} \\
Z_{F}
\end{array}\right\}=[M]^{-1} \cdot\left(\left\{\begin{array}{c}
-b_{1} \\
-b_{2} \\
-b_{3} \\
-b_{4}
\end{array}\right\}+\left\{\begin{array}{c}
b_{1 \alpha} \\
b_{2 \alpha} \\
b_{3 \alpha} \\
b_{4 \alpha}
\end{array}\right\} \cdot \frac{1}{\operatorname{tg}^{2} \alpha}\right)
$$

Funktionen der mittleren Diagonalenneigung $\alpha$. Sie können wie folgt angeschrieben werden:

$$
\lambda=\lambda_{0}-\lambda_{\alpha} \cdot \frac{1}{\operatorname{tg}^{2} \alpha}
$$

und

$$
\begin{aligned}
& z_{D}=z_{D O}+z_{D \alpha} \cdot \frac{1}{\operatorname{tg}^{2} \alpha} \\
& Z_{E}=z_{E O}+z_{E \alpha} \cdot \frac{1}{\operatorname{tg}^{2} \alpha} \\
& Z_{F}=z_{F O}+z_{F \alpha} \cdot \frac{1}{\operatorname{tg}^{2} \alpha}
\end{aligned}
$$


Für den Spezialfall reiner Längsbeanspruchung $\left(Q_{y}=Q_{z}=T_{w}=0\right)$ verschiwinden in diesen Ausdrücken jeweils die zweiten Glieder, welche die aus der Schubbeanspruchung resultierenden Anteile darstellen. Somit entspricht $\lambda_{0}$ dem für $Q_{y}=Q_{z}=T_{w}=0$ gültigen Widerstandsfaktor $\lambda$.

\section{Dritter Schritt:}

Nun braucht man nur noch die im ersten und zweiten Schritt gelösten Unterprogramme miteinander zu koppeln. Im Falle eines kombinierten Mechanismus müssen die beiden für den Widerstandsfaktor $\lambda$ erhaltenen Ausdrücke (78) und (82) übereinstimmen.

$$
\bar{\lambda} \cdot \frac{1}{|\operatorname{tg} \alpha|}=\lambda_{0}-\lambda_{\alpha} \cdot \frac{1}{\operatorname{tg}^{2} \alpha}
$$

Die Verschmelzung der beiden Unterprogramme ist damit vollzogen. Aus der obigen Gleichung lässt sich die bis anhin unbekannte Diagonalenneigung $\alpha$ bestimmen,

$$
\frac{1}{|\operatorname{tg} \alpha|}=-\frac{\bar{\lambda}}{2 \cdot \lambda_{\alpha}}+\sqrt{\left(\frac{\bar{\lambda}}{2 \cdot \lambda_{\alpha}}\right)^{2}+\frac{\lambda_{0}}{\lambda_{\alpha}}}
$$

wobei folgende Begrenzung des Winkels $\alpha$ zu beachten ist.

$$
0,5 \leq \frac{1}{|\operatorname{tg} \alpha|} \leq 2,0
$$

Bewegt sich die Diagonalenneigung $\alpha$ innerhalb der angegebenen Grenzen, so tritt ein kombinierter Mechanismus ein. Die Gleichungen (78) und (82) liefern dann denselben Widerstandsfaktor $\lambda$. Kommt hingegen eine der beiden Umlagerungsgrenzen ins Spiel, so entwickelt sich die Richtigkeit dieser Grenzen vorausgesetzt, entweder ein Längs- oder ein Schubmechanismus. Dementsprechend werden sich die aus (78) und (82) erhaltenen Widerstandsfaktoren unterscheiden. Massgebend ist der kleinere der beiden Werte, so dass in jedem Falle folgende Beziehung gilt.

$$
\lambda=\operatorname{Min} \cdot\left\{\begin{array}{l}
\bar{\lambda} \cdot \frac{1}{\mid \operatorname{tg} \alpha} \\
\lambda_{0}-\lambda_{\alpha} \cdot \frac{1}{\operatorname{tg}^{2} \alpha}
\end{array}\right\}
$$

Damit hat man, falls auch die nach (83) zu ermittelnden Gurtstabkräfte die Plastizitätsbedingungen erfüllen, den Bruchwiderstand des Querschnittes gefunden. 
Spezialfall reiner Schubbeanspruchung:

Für den Fall $N=M_{y}=M_{z}=M_{\omega}=0$ ist das für die Längsbeanspruchung gültige Programm in Bild 61 wie folgt umzuschreiben:

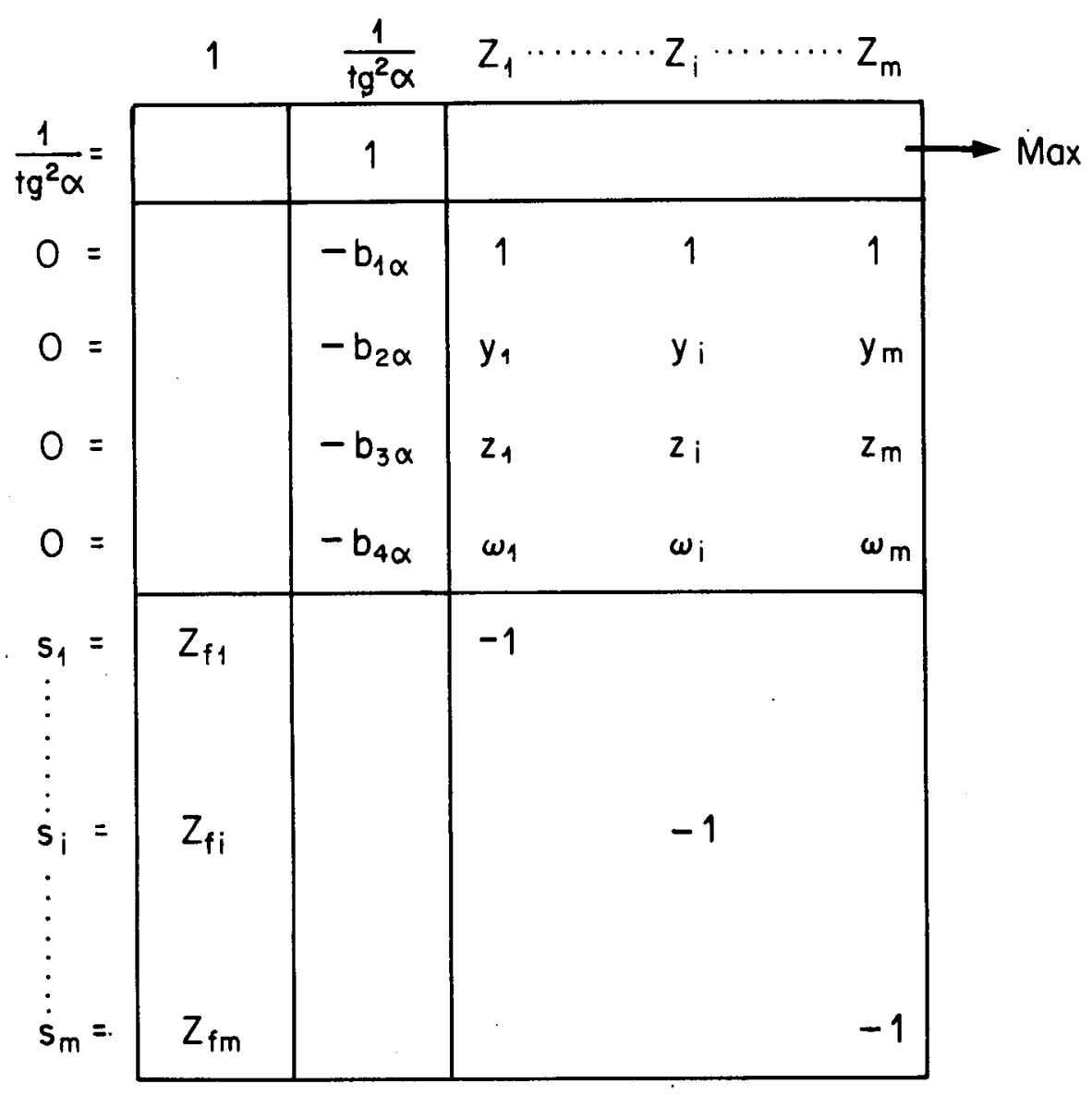

Bild 62

Die Zielfunktion $\frac{1}{\operatorname{tg}^{2} \alpha} \rightarrow$ Maximum ist gleichbedeutend mit der Frage nach dem grössten Widerstandsfaktor $\lambda$, denn der Betrag der Schubbeanspruchung $(\lambda)$ ist nach Gleichung (78) eine direkte Funktion von $\frac{1}{|\operatorname{tg} a|}$ und damit von $\frac{1}{\operatorname{tg}^{2} \alpha}$. Physikalisch gesehen bedeutet dies, dass nach der flachsten Diagonalenneigung $\alpha$ gesucht wird, welche aufgrund der vorhandenen Längsarmierung noch möglich ist.

Bei genau gleichem Vorgehen wie bei einem reinen Längsmechanismus, wo alle ausser drei Gurtstäben $D, E$ und $F$ ihre Fliessgrenze erreichen, führt das angegebene Programm zu einem linearen Gleichungssystem. 


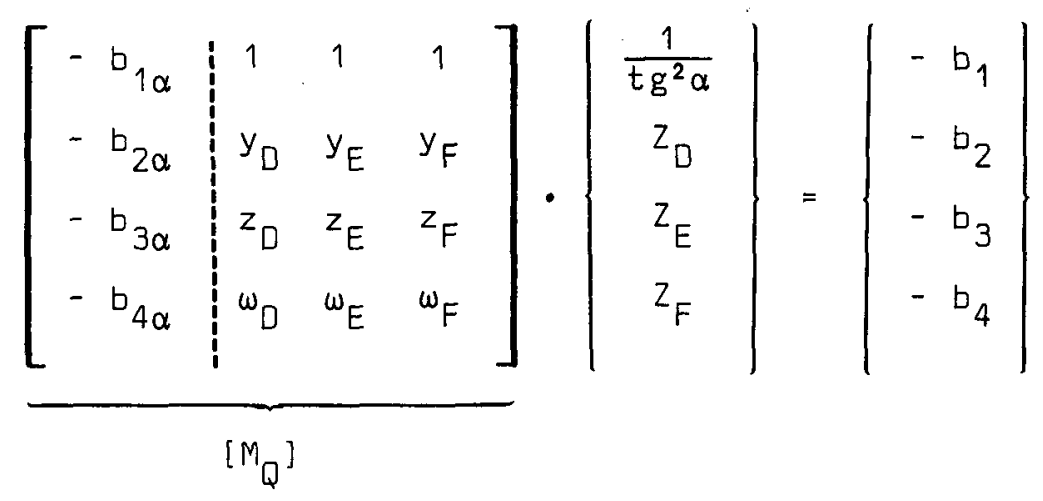

oder

$$
\left|\begin{array}{c}
\frac{1}{\operatorname{tg}^{2} \alpha} \\
z_{D} \\
z_{E} \\
z_{F}
\end{array}\right|=\left[M_{Q}\right]^{-1} \cdot\left|\begin{array}{c}
-b_{1} \\
-b_{2} \\
-b_{3} \\
-b_{4}
\end{array}\right|
$$

Wie man sieht, lässt sich die Diagonalenneigung a direkt aus diesem Gleichungssystem bestimmen. Kennt man die Diagonalenneigung, so ist der Widerstandsfaktor gegeben,

$$
\lambda=\bar{\lambda} \cdot \frac{1}{|\operatorname{tg} \alpha|}
$$

wobei der Umlagerungswinkel $\alpha$ wie folgt zu begrenzen ist.

$$
\frac{1}{|\operatorname{tg} \alpha|} \leq 2,0
$$

Erreicht die Funktion $\frac{1}{\operatorname{tg} \alpha}$ einen Wert, welcher die untere Grenze 0,5 unterschreitet, so ist der berechnete wert und nicht der Grenzwert 0,5 in Gleichung (87) einzusetzen, weil sonst - infolge der im Tableau des Bildes 62 enthaltenen Annahme fliessender Bügel (erster Schritt) - die aus der Schubbeanspruchung resultierenden Längskräfte nicht mehr aufgenommen werden könnten.

Somit entfällt für eine reine Schubbeanspruchung die Kontrolle der Umlagerungsgrenze $\frac{1}{|\operatorname{tg} \alpha|} \geq 0,5$ bei der numerischen Bestimmung des Querschnitts- 
widerstandes. Liegt der berechnete Wert unter 0,5, so ist lediglich daran zu denken, dass die Bügel wegen der in Wirklichkeit beschränkten Diagonalenneigung $\alpha$ ihre fliessgrenze nicht mehr erreichen.

\subsubsection{Numerisches Beispiel}

Zur Illustration des vorher beschriebenen Lösungsweges wird folgendes Beispiel durchgerechnet.

\section{Gegeben:}

Querschnitt:

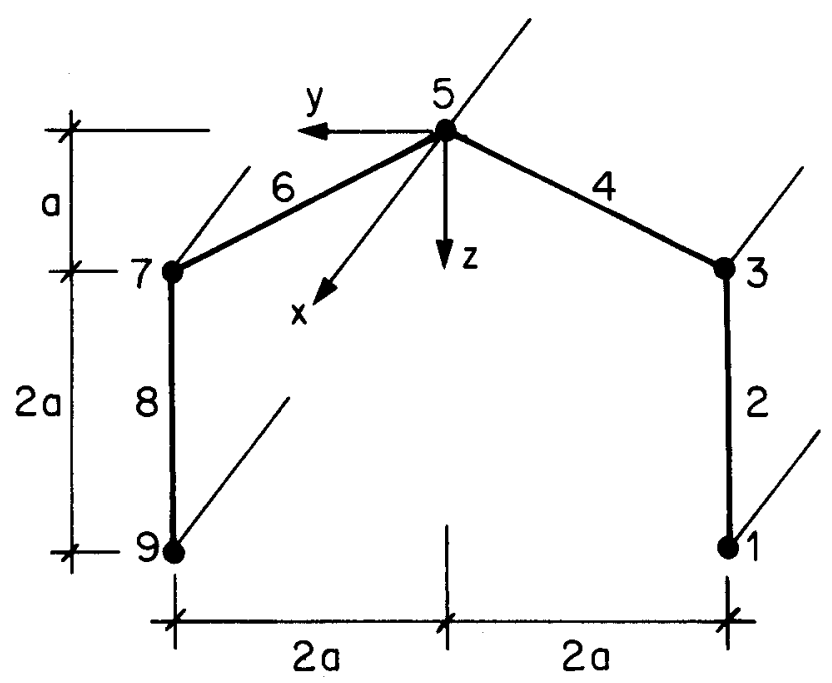

Bild 63

Fliesskräfte der Armierung:

$$
\begin{aligned}
& z_{f 1}=z_{f g}=2 \cdot z_{f} \\
& z_{f 3}=z_{f 5}=z_{f 7}=z_{f} \\
& \frac{B_{f k}}{t_{k}}=\frac{0,20}{a} \cdot z_{f} \text { für allek }
\end{aligned}
$$


Schnittkräfte:

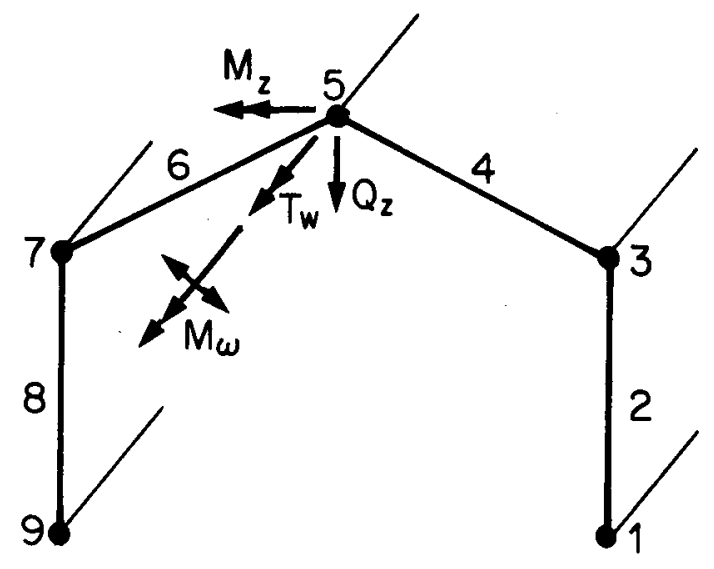

Bild 64

Grösse resp. Verhältnis der Schnittkräfte:

$$
\begin{array}{ll}
M_{z}=Z_{f} \cdot a & Q_{z}=0,10 \cdot Z_{f} \\
M_{w}=Z_{f} \cdot a^{2} & T_{w}=0,10 \cdot z_{f} \cdot a
\end{array}
$$

Gesucht: Plastischer Querschnittswiderstand $\lambda \cdot\left(M_{z}, M_{\omega}, Q_{z}, T{ }_{w}\right)$

Koordinaten:

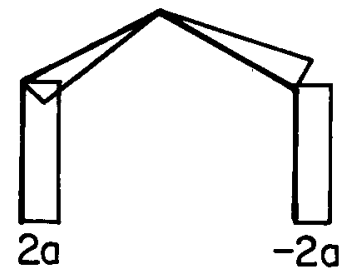

(y)

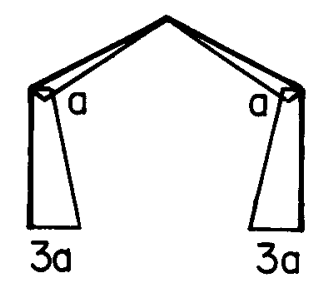

(2)

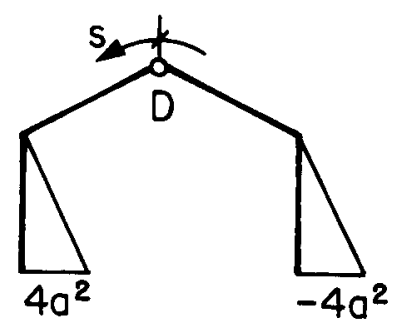

(w)

\section{Erster Schritt:}

Annahme eines Schubmechanismus:

$$
\begin{aligned}
& \bar{Q}_{6}=\bar{Q}_{f 6}=B_{f 6} \cdot \frac{a_{6}}{t_{6}}=0,20 \cdot \sqrt{5} \cdot z_{f} \\
& \bar{Q}_{8}=\bar{Q}_{f 8}=B_{f 8} \cdot \frac{a_{8}}{t_{8}}=0,40 \cdot z_{f}
\end{aligned}
$$

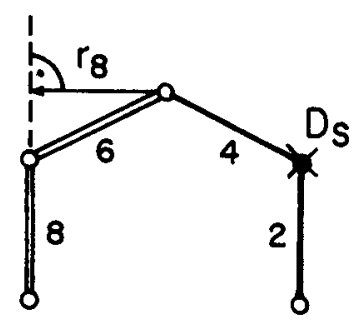




$$
\begin{aligned}
& q_{1}=\sum_{k=f k} \bar{Q}_{k} \cdot \cos \delta_{k}=0,40 \cdot z_{f} \\
& q_{2}=\sum_{k=f k} \bar{Q}_{k} \cdot \sin \delta_{k}=0,60 \cdot z_{f} \\
& q_{3}=\sum_{k=f k} \bar{Q}_{k} \cdot r_{k}=0,80 \cdot z_{f} \cdot a
\end{aligned}
$$

Gleichgewichtsbedingungen für die Schubkräfte:

$$
\begin{aligned}
& Q_{4} \cdot \frac{2}{\sqrt{5}}=-0,40 \cdot z_{f} \cdot \frac{1}{|\operatorname{tg} \alpha|} \\
& -\lambda \cdot 0,10 \cdot z_{f}-Q_{2}-Q_{4} \cdot \frac{1}{\sqrt{5}}=-0,60 \cdot z_{f} \cdot \frac{1}{|\operatorname{tg} \alpha|} \\
& -\lambda \cdot 0,10 \cdot z_{f} \cdot a+Q_{2} \cdot 2 a \quad=-0,80 \cdot z_{f} \cdot a \cdot \frac{1}{|\operatorname{tg} \alpha|} \\
& \rightarrow \\
& \lambda=\bar{\lambda} \cdot \frac{1}{|\operatorname{tg} \alpha|}=8 \cdot \frac{1}{|\operatorname{tg} \alpha|} \\
& Q_{2}=\bar{Q}_{2} \cdot \frac{1}{|\operatorname{tg} \alpha|}=0 \\
& Q_{4}=\bar{Q}_{4} \cdot \frac{1}{|\operatorname{tg} \alpha|}=0,20 \cdot \sqrt{5} \cdot z_{f} \cdot \frac{1}{\mid \operatorname{tg} \alpha}
\end{aligned}
$$

Plastizitätsbedingungen für die Bügel:

$$
\left.\begin{array}{l}
\left|\bar{Q}_{2}\right|=0<\bar{Q}_{f 2}=0,40 \cdot z_{f} \\
\left|\bar{Q}_{4}\right|=0,20 \cdot \sqrt{5} \cdot z_{f}=\bar{Q}_{f 4}=0,20 \cdot \sqrt{5} \cdot z_{f}
\end{array}\right\} \text { i.o. }
$$

\section{Zweiter Schritt:}

Längsbeanspruchung aus $Q_{k}$ für $\frac{1}{|\operatorname{tg} \alpha|}=1$ :

$$
\begin{aligned}
& b_{1 \alpha}=\sum_{k=2}^{n}\left|\bar{Q}_{k}\right|=1,294 \cdot z_{f} \\
& b_{2 \alpha}=\sum_{k=2}^{n}\left|\bar{Q}_{k}\right| \cdot y_{k}=0,80 \cdot z_{f} \cdot a \\
& b_{3 \alpha}=\sum_{k=2}^{n}\left|\bar{Q}_{k}\right| \cdot z_{k}=1,247 \cdot z_{f} \cdot a \\
& b_{4 \alpha}=\sum_{k=2}^{n}\left|\bar{Q}_{k}\right| \cdot \omega_{k}=0,80 \cdot z_{f} \cdot a^{2}
\end{aligned}
$$


Annahme eines Längsmechanismus:

$$
\begin{aligned}
& z_{3}=z_{f 3}=z_{f} \\
& z_{g}=z_{f g}=2 \cdot z_{f}
\end{aligned}
$$
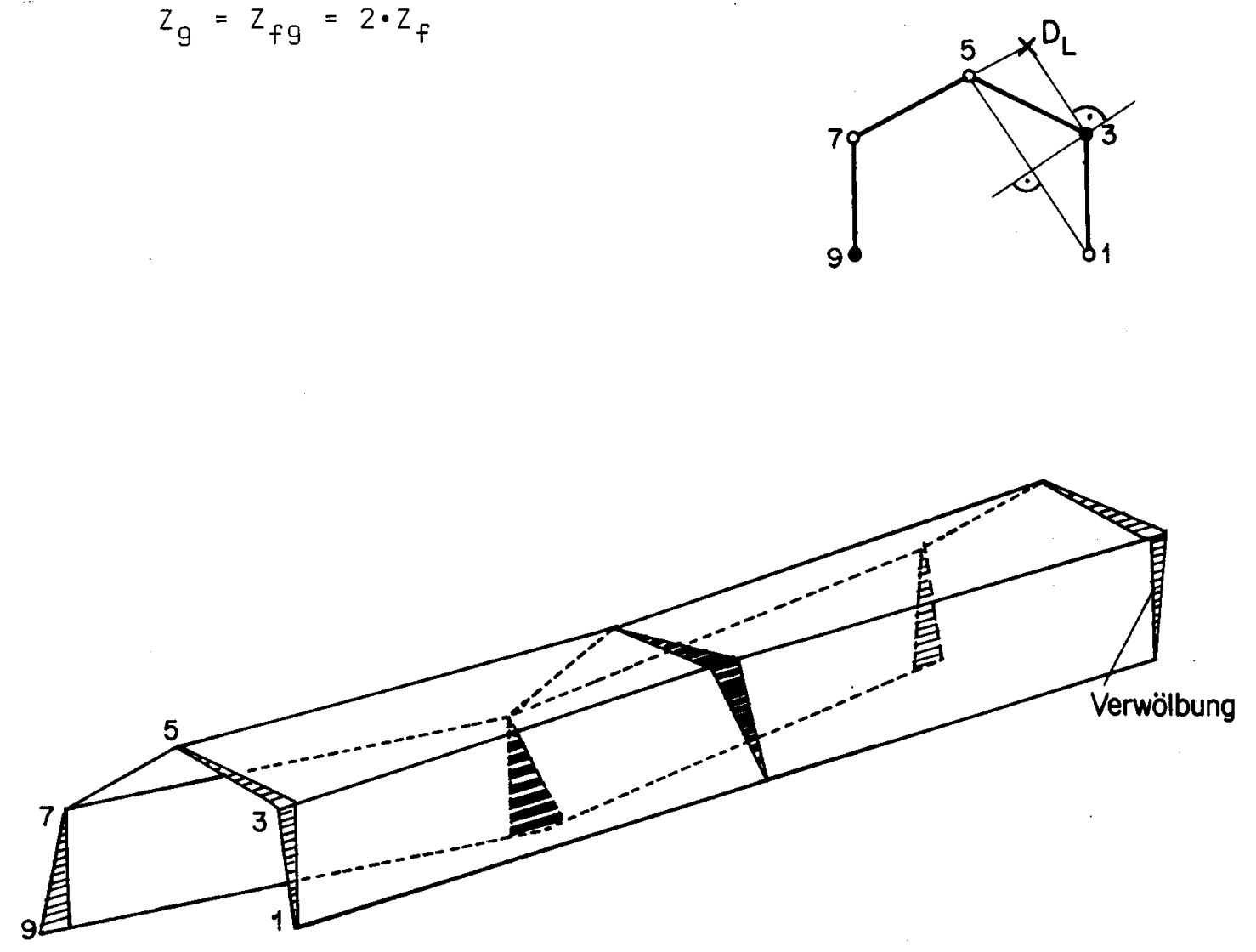

Bild 67

$$
\begin{aligned}
& b_{1}=\sum_{i=f i} z_{i}=3 \cdot z_{f} \\
& b_{2}=\sum_{i=f i} z_{i} \cdot y_{i}=2 \cdot z_{f} \cdot a \\
& b_{3}=\sum_{i=f i} z_{i} \cdot z_{i}=7 \cdot z_{f} \cdot a \\
& b_{4}=\sum_{i=f i} z_{i} \cdot w_{i}=8 \cdot z_{f} \cdot a^{2}
\end{aligned}
$$


Gleichgewichtsbedingungen für die Längskräfte:

$$
\left[\begin{array}{cccc}
0 & 1 & 1 & 1 \\
0 & -2 \cdot a & 0 & 2 \cdot a \\
-z_{f} \cdot a & 3 \cdot a & 0 & a \\
-z_{f} \cdot a^{2} & -4 \cdot a^{2} & 0 & 0
\end{array}\right] \cdot\left\{\begin{array}{l}
\lambda \\
z_{1} \\
z_{5} \\
z_{7}
\end{array}\right\}=\left\{\begin{array}{l}
-3 \cdot z_{f} \\
-2 \cdot z_{f} \cdot a \\
-7 \cdot z_{f} \cdot a \\
-8 \cdot z_{f} \cdot a^{2}
\end{array}\right\}+\left\{\begin{array}{l}
1,294 \cdot z_{f} \\
0,80 \cdot z_{f} \cdot a \\
1,247 \cdot z_{f} \cdot a \\
0,80 \cdot z_{f} \cdot a^{2}
\end{array}\right\} \cdot \frac{1}{\operatorname{tg}^{2} a}
$$

[M]

$$
[M]^{-1}=\left[\begin{array}{cccc}
0 & \frac{1}{4 \cdot Z_{f} \cdot a} & \frac{-1}{2 \cdot Z_{f} \cdot a} & \frac{-1}{2 \cdot z_{f} \cdot a^{2}} \\
0 & \frac{-1}{16 \cdot a} & \frac{1}{8 \cdot a} & \frac{-1}{8 \cdot a^{2}} \\
1 & \frac{-3}{8 \cdot a} & \frac{-1}{4 \cdot a} & \frac{1}{4 \cdot a^{2}} \\
0 & \frac{7}{16 \cdot a} & \frac{1}{8 \cdot a} & \frac{-1}{8 \cdot a^{2}}
\end{array}\right]
$$

$$
\begin{aligned}
& \lambda=\lambda_{0}-\lambda_{\alpha} \cdot \frac{1}{\operatorname{tg}^{2} \alpha}=7-0,824 \cdot \frac{1}{\operatorname{tg}^{2} \alpha} \\
& z_{1}=z_{10}+z_{1 \alpha} \cdot \frac{1}{\operatorname{tg} \alpha}=z_{f} \cdot\left(0,25+0,005 \cdot \frac{1}{\operatorname{tg}^{2} \alpha}\right) \\
& z_{5}=z_{50}+z_{5 \alpha} \cdot \frac{1}{\operatorname{tg}^{2} \alpha}=z_{f} \cdot\left(-2,50+0,882 \cdot \frac{1}{\operatorname{tg}^{2} \alpha}\right) \\
& z_{7}=z_{70}+z_{7 \alpha} \cdot \frac{1}{\operatorname{tg}^{2} \alpha}=z_{f} \cdot\left(-0,75+0,406 \cdot \frac{1}{\operatorname{tg}^{2} \alpha}\right)
\end{aligned}
$$

\section{Dritter Schritt:}

Mittlere Diagonalenneigung:

$$
\begin{aligned}
\frac{1}{\operatorname{tg} \alpha} & =-\frac{\bar{\lambda}}{2 \cdot \lambda_{\alpha}}+\sqrt{\left(\frac{\bar{\lambda}}{2 \cdot \lambda_{\alpha}}\right)^{2}+\frac{\lambda_{0}}{\lambda_{\alpha}}}= \\
& =-\frac{8}{2 \cdot 0,824}+\sqrt{\left(\frac{8}{2 \cdot 0,824}\right)^{2}+\frac{7}{0,824}} \\
\frac{1}{\operatorname{tg} \alpha} & =0.808
\end{aligned}
$$


Kontrolle der Diagonalenneigung:

$$
0,5 \leq \frac{1}{\operatorname{tg} \alpha \mid} \leq 2,0 \quad \text { i. } 0
$$

Resultat:

$$
\begin{aligned}
& \lambda=\bar{\lambda} \cdot \frac{1}{|\operatorname{tg} \alpha|}=6,46 \\
& \lambda=\lambda_{0}-\lambda_{\alpha} \cdot \frac{1}{\operatorname{tg}^{2} \alpha}=6,46
\end{aligned}
$$

$\lambda=6,46$

$==\mathbf{=}=\mathbf{=}=$

Plastizitätsbedingungen für die Gurtstäbe:

$$
\begin{aligned}
& z_{1}=0,25 \cdot z_{f}<z_{f 1}=2 \cdot z_{f} \\
& z_{5}=-1,92 \cdot z_{f}<z_{f 5}=z_{f} \\
& z_{7}=-0,48 \cdot z_{f}<z_{f 7}=z_{f}
\end{aligned}
$$

i. o.

\subsection{Anwendungsgrenzen}

Das angenommene Bruchmodell gilt nur für unterarmierte Querschnitte, deren Bruchwiderstand durch das Fliessen der Armierung bestimmt wird. Dies setzt eine entsprechende konstruktive Ausbildung der Tragwerke voraus, welche ein lokales Versagen wie z.B. ein Verankerungsbruch oder ein Ausbrechen der Betondiagonalen in den Querschnittsecken ausschliesst. Ausserdem ist darauf zu achten, dass weder der Beton für ein Versagen massgebend wird, noch die Armierung beim ersten Anriss des Betons durchreisst. Derartige Sprödbrüche sind zu vermeiden.

a) Bruch bei Bildung der ersten Risse durch Zerreissen der Armierung.

b) Stauchungsbruch eines auf Druck beanspruchten Gurtstabes vor dem Fliessen der Armierung.

c) Stauchungsbruch der Betondruckdiagonalen vor dem Fliessen der Armierung.

Um ein Zerreissen der Armierung beim Auftreten der ersten Betonrisse zu 
verhindern, muss die beim Reissen freiwerdende Energie durch die Armierung aufgenommen werden können. Da sich Stahlbeton bis zum Eintreten der Risse ungefähr wie ein homogener elastischer Baustoff verhält, lässt sich die erforderliche Minimalarmierung mit Hilfe der Elastizitätstheorie bestimmen. Eine Grössenordnung gibt die bekannte Minimalarmierung für reine Biegung.

Für das Versagen druckbeanspruchter Gurtstäbe sind die mitwirkenden Betondruckzonen von Bedeutung. Ihre Ausdehnung ist, weil der Bruch durch Stahlfliessen verursacht werden soll, derart zu beschränken, dass die für den Mechanismus massgebenden Gurtstäbe tatsächlich ins Fliessen kommen $\left(\varepsilon_{x}=\frac{\sigma_{f}}{E_{e}} \simeq 3 \% 0\right)$. Als Grenzfall interessiert jener Zustand, bei dem gleichzeitig mit Beginn des Mechanismus die Bruchgrenze eines auf Druck beanspruchten Gurtstabes erreicht wird. Die Randbruchstauchung des Betons kann dabei wie im Falle reiner Biegung auf etwa $\varepsilon_{x}=-3 \%$ fixiert werden, so dass man für den erwähnten Grenzzustand folgende Dehnungs- und Spannungsverteilung erhält.

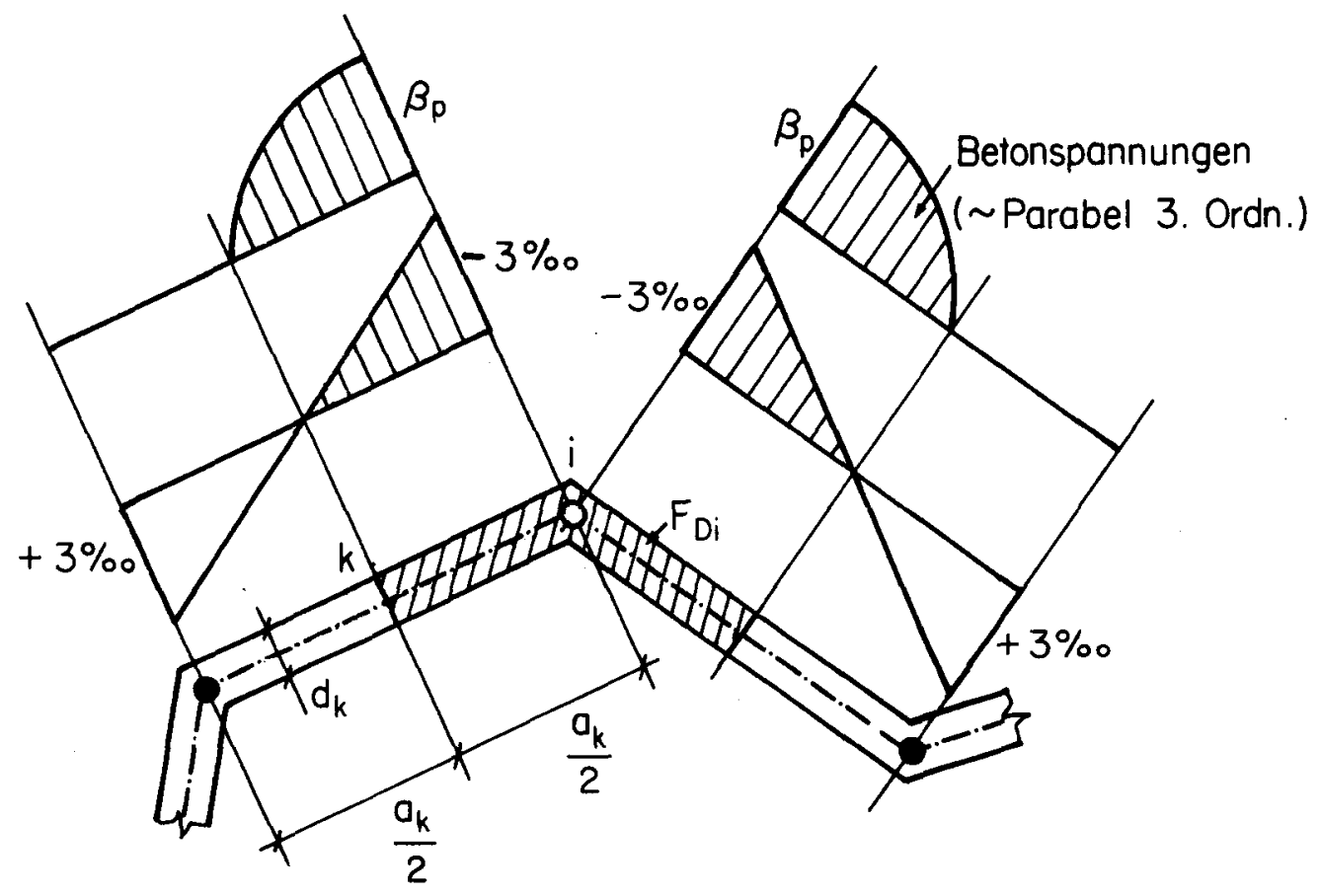

Bild 68

Mit $\mathrm{Hilfe}$ dieser Skizze, worin $\beta_{p}$ die rechnerische Prismendruckfestigkeit des Betons bedeutet, lassen sich die Druckbruchkräfte der Gurtstäbe abschätzen. Sie dürfen, wenn ein Versagen des Betons vor dem Fliessen der massgebenden Zugarmierung ausgeschlossen werden soll, bei keinem Gurtstab überschritten werden. 


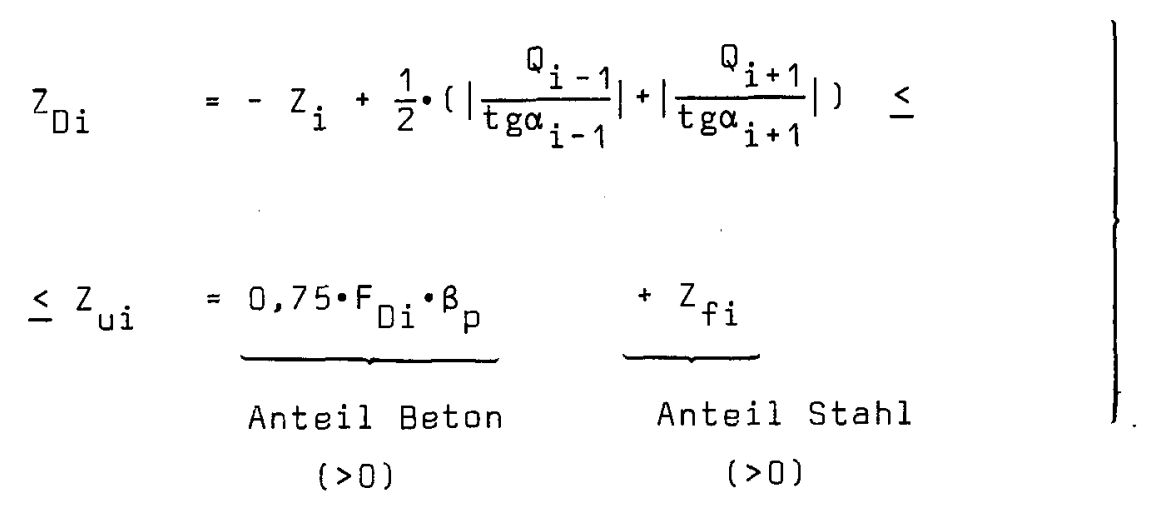

Da sich die Druckzone $F_{D i}$ auf die angrenzenden Querschnittscheiben erstreckt, wird sie nicht nur durch die Gurtstabkraft $Z_{i}$ sondern auch durch die Schubkräfte der Querschnittscheiben beansprucht. Für die dargestellte Ausdehnung der Druckzone erhält man infolge Schub eine zusätzliche Beanspruchung des Betons in Längsrichtung, wie sie in (89) angegeben ist. Sie erklärt sich aus der Wirkungsweise einer schubbeanspruchten Querschnittscheibe (Abschnitt 5.1).

Führt die Kontrolle der beim Widerstandsfaktor $\lambda$ vorhandenen Druekkräfte $Z_{D i}(\lambda)$ zu einer Verletzung der Ungleichung (89), so muss mit einem vorzeitigen Sprödbruch gerechnet werden. Der Widerstandsfaktor $\lambda$, welcher unter Voraussetzung duktilen Bruchverhaltens (Stahlfliessen) ermittelt wurde, liegt dann über dem effektiven Wert $\lambda_{\text {eff' }}$ da die massgebende Längszugarmierung bei Einhaltung der Bedingung (89) nicht mehr ins Fliessen kommen kann. In diesem Falle lässt sich der Querschnittswiderstand folgendermassen abschätzen,

$$
\begin{aligned}
& \lambda_{\text {eff }} \simeq \lambda \cdot\left|\frac{z_{u i}}{z_{D i}(\lambda)}\right|_{\min } \\
& \text { für } z_{D i}(\lambda) \geq z_{u i}
\end{aligned}
$$

indem der Widerstandsfaktor $\lambda$ derart vermindert wird, bis der am meisten gefährdete Gurtstab $i$ seine Bruchgrenze $Z_{u i}$ nicht mehr überschreitet. Für $\lambda_{\text {eff }}$ ist damit neben den Gleichgewichts- und Plastizitätsbedingungen auch die Bedingung (89) erfüllt.

In diesem Zusammenhang ist darauf hinzuweisen, dass die anfallende Druckbeanspruchung nach Abschnitt 5.5 und 5.6 (duktiles Bruchverhalten) innerhalb dreier Gurtstäbe aufgenommen werden muss, da bei einem Längsmechanismus alle andern Gurtstäbe fliessen. Aus diesem Grunde darf der Bereich der erwähnten drei Gurtstäbe nicht zu klein oder, was auf dasselbe herauskommt, die Anzahl der für die Berechnung angenommenen Gurtstäbe nicht zu gross gewählt werden. 


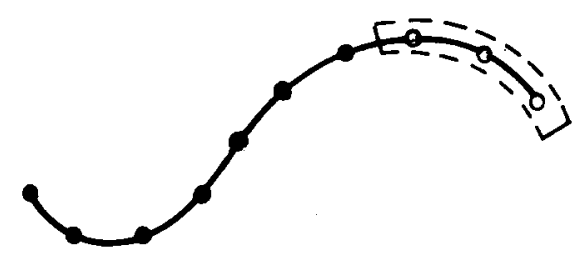

- : fliessende Gurtstäbe

- : starre Gurtstäbe

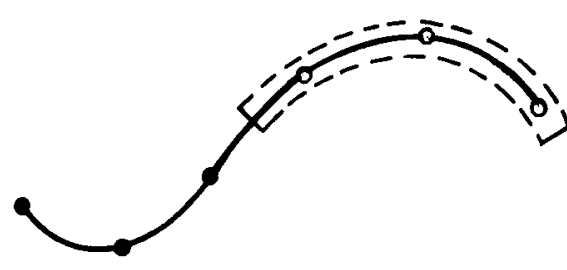

[-] : grosstmöglicher Druckbereich

von Gurtstäben die Bedingung (89) viel eher massgebend wird, was bei der zur Verfügung stehenden Berechnungsmethode (Abschnitt 5.6) keineswegs erwünscht ist. In normalen fällen dürfte man optimale Werte erhalten, wenn für den Hauptquerschnitt (vgl. Abschnitt 2.4) vier bis sechs Gurtstäbe angenommen werden.

Um ein Versagen der Betondruckdiagonalen vor einem Fliessen der Armierung zu verhindern, wird wie im Falle des Querkraftschubes mit einer oberen Schubspannungsgrenze $\tau_{u}$ gearbeitet. Für dünnwandige Querschnitte entspricht dieser Wert etwa der oberen Schubspannungsgrenze eines auf Schub beanspruchten Balkensteges. Es gilt:

$$
\tau_{k}=\frac{Q_{k}}{a_{k} \cdot d_{k}} \leq \tau_{u}
$$

Wird diese obere Schubspannungsgrenze beim Erreichen des Widerstandfaktors $\lambda$ irgendwo überschritten, so ergibt sich eine ähnliche Abminderung des Widerstandfaktors gegenüber dem berechneten wert $\lambda$ wie im Falle eines Gurtstab-Stauchungsbruches.

$$
\begin{aligned}
& \lambda_{\text {eff }} \simeq \lambda \cdot\left|\frac{\tau_{u}}{\tau_{k}(\lambda)}\right|_{\min } \\
& \text { für } \tau_{k}(\lambda) \geq \tau_{u}
\end{aligned}
$$

Für gedrungene Querschnitte, welche bisher nicht behandelt wurden, scheinen abschliessend einige Bemerkungen angebracht. Bei solchen Querschnitten kann die Plattenwirkung nicht mehr ohne weiteres vernachlässigt werden. Sie beeinflusst das Tragverhalten, indem sie zu einer Erhöhung des Bruchwider- 
standes führt. Wird ihr Anteil am Bruchwiderstand für die Bemessung ausgenützt, so treten bei gedrungener Querschnittsausbildung schon im Gebrauchszustand grosse Risse auf, welche auf die ungleiche Dehnungsverteilung über die Querschnittsdicke (Plattenverformung) zurückzuführen sind. Das heisst, dass sich die Plattenwirkung, obwohl sie den Bruchwiderstand beachtlich erhöhen kann, ungünstig auf das Verformungs- und Rissverhalten im Gebrauchszustand auswirkt. Aus diesem Grunde darf die Plattenwirkung nach den bisherigen Untersuchungen (z.B. [12]) nur bei eingehender Prüfung des Gebrauchszustandes voll in Rechnung gestellt werden. Wird sie für eine Bemessung vernachlässigt, so erhält man in jedem falle neben einer ausreichenden Bruchsicherheit auch ein vernünftiges Verformungs- und Rissverhalten im Gebrauchszustand.

Infolge der Plattenverformung treten bei gedrungenen Querschnitten sehr hohe Betonbeanspruchungen auf. So werden die Druck-Gurtstäbe und die Betondruckdiagonalen neben der zentrischen Normalkraft nach dem Fachwerkmodell auch durch eine Krümmung beansprucht. Demzufolge spielen die aus der Plattenverformung resultierenden Betonstauchungen eine bedeutende Rolle für die Art des eintretenden Bruches. Wie leicht einzusehen ist, wird für bestimmte Stahldehnungen (z.B. Fliessbeginn) die Verdrehung und damit die Plattenverformung umso kleiner, je weiter die einzelnen Querschnittscheiben auseinander liegen, d.h. je grösser die Aussenabmessungen eines Querschnittes sind. Die durch die Plattenverformung bedingten Betonrandstauchungen vergrässern sich mit zunehmender Wandstärke, so dass die Gedrungenheit eines Querschnittes, welche durch das Verhältnis der Wandstärke zu den Abmessungen des Hauptquerschnittes ausgedrückt werden kann, eine Grössenordnung der zu erwartenden Betonbeanspruchung gibt.
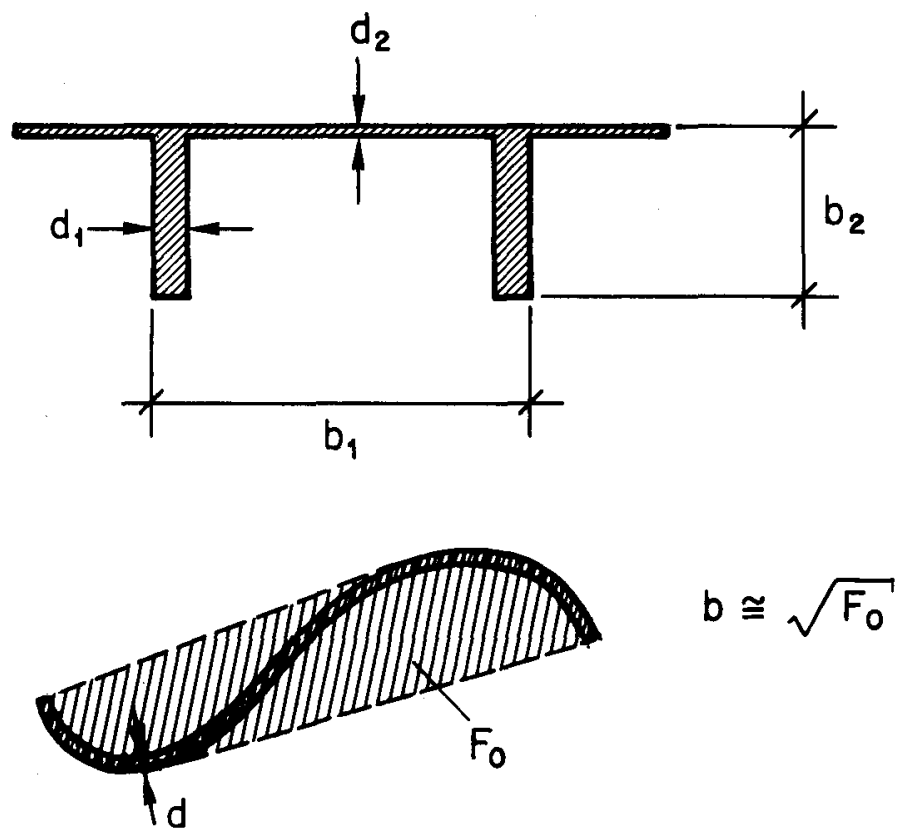
Reduziert man nun die in Rechnung zu stellende Wandstärke auf ein vernünftiges Mass, z.B.,

$$
\left(\frac{d}{b}\right)_{\max }=\frac{1}{8}
$$

so trägt man den zusätzlichen Betonbeanspruchungen aus der Plattenverformung auf einfachste Weise Rechnung. Bei gedrungenen Querschnitten dürfte kein unerwarteter Sprödbruch eintreten, solange die zentrische Beanspruchung unter Verwendung der reduzierten Wandstärke (93) die in (89) und (91) angegebenen Grenzen nicht überschreitet. 
Das Ziel der vorliegenden Arbeit ist die Bestimmung der Traglast von beliebig belasteten Stäben mit dünnwandigen offenen Querschnitten.

Zunächst werden die grundlegenden statischen und kinematischen Beziehungen hergeleitet. Dabei findet man, dass in den Gleichgewichtsbedingungen eine formelle Analogie zwischen Wölbtorsion und Balkenbiegung besteht. Folglich kann für die statischen Beziehungen der Wölbtorsion auf die Biegetheorie von Balken zurückgegriffén werden.

Es wird gezeigt, dass die Bedeutung des Schubmittelpunktes für die Torsion genauso wie diejenige des Schwerpunktes für die Biegung in der Elastizitätstheorie begründet ist. Folglich verliert der schubmittelpunkt, um den sich ein elastischer Stab bei reiner Torsion verdreht, im plastischen Zustand seine Bedeutung.

Weil die Traglast eines Stabtragwerkes von den Querschnittswiderständen und der Lage der plastifizierten Querschnitte beim Bruchmechanismus abhängt, wird die Bestimmung der Querschnittswiderstände zur eigentlichen Hauptaufgabe, die mit Hilfe der beiden Grenzwertsätze der Plastizitätstheorie gelöst wird.

Zunächst werden Träger aus homogenen isotropen Materialien wie z.B. Stahl untersucht. Der Querschnitt wird zwecks einfacher mathematischer Behandlung in einzelne Elemente unterteilt und deren Fliessbedingungen linearisiert. So erhält man den Querschnittswiderstand als Lösung linearer Programme, wobei der statische Grenzwertsatz in Form einer Maximumsaufgabe und der kinematische in Form einer Minimumsaufgabe der linearen Programmierung formuliert werden kann. Da beide Programme wegen ihrer Dualität denselben Querschnittswiderstand liefern, spielt es keine Rolle, welches der beiden Programme zur Lösung der Aufgabe verwendet wird.

Zur Beschreibung des Verhaltens von Stahlbeton- und Spannbetonträgern wird ein räumliches Fachwerkmodell mit den Längseisen als Gurtungen, den Bügeln als Pfosten und den Betondruckdiagonalen als Streben angenommen. Es ergeben sich nach beiden Grenzwertsätzen der Plastizitätstheorie wegen der variablen Neigung der Betondruckdiagonalen nichtlineare Programme, welche zueinander dual sind und demzufolge denselben Querschnittswiderstand liefern. Nach einer eingehenden Beschreibung der möglichen Bruchmechanismen wird eine einfache Näherungsmethode zur Lösung des nichtlinearen Maximumproblems entwickelt. Ein Beispiel veranschaulicht den Lösungsweg und zeigt die Effizienz der angegebenen Methode. 
RESUME

Le but de ce travail est la détermination de la charge de rupture des poutres de section ouverte à parois minces sousmises à des charges quelconques.

Premièrement les équations fondamentales statiques et cinématiques sont dérivées. Il se trouve dans les relations statiques une analogie mathématique entre la torsion due au gauchissement empêché et la flexion. C'est-à-dire, que les équations statiques pour la torsion due au gauchissement empêché peuvent être formulées selon la théorie de la flexion des barres.

On montre que le centre de cisaillement a pour la torsion la même signification que le centre de gravité pour la flexion dans le domaine élastique. Dans le domaine plastique, le centre de cisaillement déterminant l'axe de rotation du domaine élastique pour la torsion pure n'a plus aucune importance.

La charge de rupture d'une structure dépend des résistances statiques des sections et de la position des sections plastifiées qui forment le mécanisme de rupture. Ainsi la détermination de la résistance statique des sections devient le problème le plus important. Il est résolu par l'application des théorèmes de l'état-limite ultime de plasticité.

Des poutres de matériau homogène et isotrope (p.ex. en acier) sont analysées. Pour la simplification du calcul la section d'une poutre est subdivisée en plusieurs éléments et leurs conditions de fluage sont linéarisées. Par cela la résistance statique est formulée sous forme des programmes linéaires. Le théorème de l'état-limite ultime pour la limite inférieure livre un programme de maximum, le théorème pour la limite supérieure un programme de minimum selon la théorie d'optimalisation. Comme les deux programmes montrent une forme duale et donnent la même solution, on peut appliquer n'importe lequel de ces deux théorèmes.

Le comportement des poutres en béton armé et précontraint est simulé au moyen d'un treillis spatial avec l'armature longitudinale comme membrures, les étriers comme montants et des bielles comprimées en béton. A cause de l'inclinaison variable des bielles, les deux théorèmes de plasticité se transforment en programmes nonlinéaires, qui montrent une forme duale et donnent par conséquent la même résistance pour la section. Après une discussion détaillée des mécanismes de rupture, un algorithme approximatif pour la solution du programme selon le théorème de l'état-limite ultime pour la limite inférieure est développé. Un example montre l'algorithme et l'efficacité de la méthode développée. 
The aim of this study is the determination of the plastic resistance of arbitrarily loaded beams with thinwalled open cross section.

The basic static and kinematic equations are first derived. Thereby it is shown that a mathematical analogy exists between warping torsion and bending. Hence the bending theory of beams can be used to formulate the static equations for warping torsion of beams with thinwalled open cross sections.

It is shown that both the shear centre and the centroid are meaningfull in their application for torsion and bending within the theory of elasticity. However in the plastic range the shear centre, which determines the axis of rotation for pure torsion in the elastic range, has no meaning.

The ultimate load of a structure depends on the plastic resistances of the cross sections and the development of plastic hinges to form a collapse mechanism. Thus the determination of the ultimate capacities of cross sections is of primary importance. This problem is solved by use of lower and upper bound theorem of plasticity.

Beams of homogeneous and isotropic (elastic-ideally plastic) materials such as steel are investigated. In order to simplify the mathematical approach the cross section of a beam is subdivided into several elements and their yield conditions are linearized. The carrying capacity can then be formulated in form of linear programs. The use of the lower bound theorem of plasticity leads to a maximum problem, the use of the upper bound theorem to a minimum problem of linear programming. Since a mathematical duality exists between the two programs they both lead to the same result.

To describe the behavior of reinforced and prestressed concrete beams a space truss model is assumed with the longitudinal bars acting as stringers, the stirrups as vertical ties and the concrete diagonals as inclined struts. Because of the variable inclination of struts the application of the upper and lower bound theorem lead to nonlinear programs. Due to their duality they give the same carrying capacity of a cross section. After a detailed discussion of failure mechanisms a simple approximate method is given for the solution of the nonlinear maximum problem. An example illustrates the algorithm showing the effectiveness of the developed method. 
A

A

A

$A_{i}$

B

$B_{k}$

$B_{f k}$

D

$D_{k}$

E

F

$F_{D i}$

$\Delta F_{i}$

G

$I_{y}, I_{z}$

$I_{\omega}$

K

$$
M_{y}, M_{z}
$$

M

N

$Q_{i}$

$Q_{k}$

$\bar{Q}_{k}$

$Q_{f k}$

$\bar{Q}_{f k}$

S

T

$T_{s}$
Koordinatenursprung des kartesischen Koordinatensystems $x$, $y, z$

Arbeit der äusseren Kräfte

Dissipationsarbeit

Arbeit der inneren Kräfte

Ursprung der Querschnittskoordinate s

Bügelzugkraft in der k-ten Querschnittscheibe

FIiesskraft eines Bügels der k-ten Querschnittscheibe

Bezugspunkt für die Torsionsbelastung

Resultierende der Betondruckspannungen in den Diagonalen

der Querschnittscheibe $k$

Elastizitätsmodul

Querschnittsfläche

Betondruckzone um den druckbruchgefährdeten Gurtstab i

(nach Abschnitt 5.7)

Querschnittsfläche des Elementes $i$ mit der Seitenlänge $\Delta s_{i}$ Schubmodul

Trägheitsmomente

Wälbkonstante

Torsionskonstante

Biegemomente

Wölbmoment

Normalkraft

Schubresultierende des Elementes i

Schubresultierende der k-ten Quersehnittscheibe

Schubresultierende für tga $\alpha_{k}=1$

Fliessschubkraft der k-ten Querschnittscheibe

Fliessschubkraft für tg $\alpha_{k}=1$

Schubfluss $\left(S=\tau_{\times s} \cdot d\right)$

Torsionsmoment

St. Venant'sches Torsionsmoment 
$T_{w}$

$z_{i}$

$\mathrm{z}_{\mathrm{k}}$

${ }^{\mathrm{Z}} \mathrm{i}$

$\mathrm{Z}_{\mathrm{fi}}$

$z_{u i}$

$a_{k}$

b

$c_{k}$

d

$d_{k}$

$f$

i

k

$m_{y}, m_{z}, m_{w}$

$\mathrm{m}_{\mathrm{x}}$

$P_{x}$

$\bar{p}_{x}$

$P_{y}, P_{z}$

r

s

$s_{i}, s_{k}$

$s_{i 1} \cdots s_{i 6}$

$t_{k}$

$u, v, w$

$x, y, z$
Wölbtorsionsmoment

Zugkraft im Element i, Zugkraft im Gurtstab i

aus der Schubbeanspruchung $Q_{k}$ resultierende Längskraft

anfallende Druckkraft im Gurtstab i (nach Abschnitt 5.7)

Fliesszugkraft des Gurtstabes i

Druckbruchkraft des Gurtstabes i

Seitenlänge der k-ten Querschnittscheibe

Aussenabmessung des Querschnittes

Verformungsparameter der k-ten Querschnittscheibe

(nominelle Rissdehnung)

Wandstärke des Querschnittes

Betonstärke der k-ten Querschnittscheibe

Tangente an den Querschnitt

Numerierung der Elemente: $i=1, \ldots, m$ (laufend),

Numerierung der Gurtstäbe: $i=1, \ldots . m$ (ungerade Zahlen)

Numerierung der Querschnittscheiben: $k=2, \ldots, n$

(gerade Zahlen)

verteilte Momentenbelastungen infolge der Längsbelastung $\bar{p}_{x}$

Torsionsbelastung $[\mathrm{mt} / \mathrm{m}]$

Integral $[t / m]$ der Längsbelastung $\overline{\mathrm{p}}_{x}$

Lärigsbelastung $\left[t / \mathrm{m}^{2}\right]$

Querbelastungen $[t / m]$

Abstand der Querschnittstangente $f$ vom Bezugspunkt der Torsionsbelastung

Querschnittskoordinate

Schlupfvariablen

Bügelabstand in der k-ten Querschnittscheibe

Verschiebungen des Querschnittes in Richtung der Koordinatenaxen $x, y$ und $z$ (ohne Schiebungsanteile)

Koordinatenaxen, Koordinaten 
$\alpha$

$\alpha_{k}$

$$
\beta_{i 1} \cdots \beta_{i 6}
$$$$
{ }_{\mathrm{B}}
$$$$
\gamma
$$$$
\gamma_{y}, \gamma_{z}
$$$$
\gamma_{\vartheta}
$$

$\delta$

$\varepsilon$

$\varepsilon_{\mathrm{B}}$

$\varepsilon_{f}$

$\varepsilon_{x}$

$\vartheta$

$\lambda$

$\bar{\lambda}, \lambda_{0}, \lambda_{\alpha}$

$\sigma$

$\sigma_{f}$

$\sigma_{x}$

$\tau$

$\tau_{u}$

$\omega$

mittlere Neigung der Betondruckdiagonalen im Fachwerkmodell

Neigung der in der k-ten Querschnittscheibe liegenden

Betondruckdiagonalen

Verformungsparameter für das Element i

Prismendruckfestigkeit des Betons

Schiebung $\left(\gamma=\gamma_{x s}\right)$

Schiebung des Querschnittes in Richtung der $y$-, bzw. z-Axe

Drehschiebung des Querschnittes um den Punkt D

Winkel zwischen der Querschnittstangente $f$ und der $y$-Axe

Dehnung

Bügeldehnung

Dehnung der Armierung bei Fliessbeginn

Längsdehnung

Verdrehung des Querschnittes um den Punkt $D$

(ohne Schiebungsanteil)

Widerstandsfaktor

Abkürzungen gemäss Abschnitt 5.6 .2

Normalspannung

Fliessspannung bzw. Streckgrenze der Armierung

Längsspannung

Schubspannung $\left(\tau=\tau_{x S}\right)$

obere Schubspannungsgrenze

sektorielle oder Wälbkoordinate 
[1]

[5].

Wlassow W.S.: "Dünnwandige elastische Stäbe", Band 1, VEB Verlag für Bauwesen Berlin, 1964.

Kollbrunner C.F./Basler K.: "Torsion", Springer Verlag Berlin/ Heidelberg/New York, 1966.

Kollbrunner C.F./Hajdin N.: "Dünnwandige Stäbe", Band 1, Springer Verlag Berlin/Heidelberg/New York, 1972.

Lundgren H.: "Cylindrical Shells", Danish Technical Press Copenhagen, 1949.

Prager W.: "Probleme der Plastizitätstheorie", Birkhäuser Verlag Basel/Stuttgart, 1955.

Thürlimann B./Ziegler H.: "Plastische Berechnungsmethoden", Eidgenössische Technische Hochschule (ETH) Zürich, 1963.

Basler K./Thürlimann B.: "Strength of Plate Girders in Bending", ASCE-Proc. No. 2913, ST6, p. 154 (Aug. 1961).

Basler K.: "Strength of Plate Girders in Shear", ASCE-Proc. No. 2967, ST7, p. 151 (Oct. 1961).

Lampert P.: "Bruchwiderstand von Stahlbetonbalken unter Torsion und Biegung", Dissertation Nr. 4445, ETH Zürich, 1970.

Stiefel E.: "Einführung in die numerische Mathematik", Teubner Verlagsgesellschaft Stuttgart, 1965.

Künzi H.P./Krelle W.: "Einführung in die mathematische Optimierung", Verlag Industrielle Organisation Zürich, 1969.

Grob J./Thürlimann ..: "Wölbtorsionsversuche an Stahlbetonbalken mit offenem Querschnitt", Bericht Nr. 6506-6, Institut für Baustatik und Konstruktion, ETH Zürich, 1974. 